\title{
Synthesis of Some New Folic Acid-Based Heterocycles of Anticipated Biological Activity
}

\author{
Ola A. Abu Ali *, Hosam A. Saad and Bodor M. A. Al Malki
}

check for

updates

Citation: Abu Ali, O.A.; Saad, H.A.; Al Malki, B.M.A. Synthesis of Some New Folic Acid-Based Heterocycles of Anticipated Biological Activity. Molecules 2021, 26, 368. https:// doi.org/10.3390/molecules26020368

Received: 17 December 2020 Accepted: 10 January 2021 Published: 12 January 2021

Publisher's Note: MDPI stays neutral with regard to jurisdictional clai$\mathrm{ms}$ in published maps and institutional affiliations.

Copyright: (C) 2021 by the authors. Licensee MDPI, Basel, Switzerland. This article is an open access article distributed under the terms and conditions of the Creative Commons Attribution (CC BY) license (https:// creativecommons.org/licenses/by/ $4.0 /)$.
Department of Chemistry, College of Science, Taif University, P.O. Box 11099, Taif 21944, Saudi Arabia; h.saad@tu.edu.sa (H.A.S.); bedoo201044@hotmail.com (B.M.A.A.M.)

* Correspondence: o.abuali@tu.edu.sa

\begin{abstract}
To date, no fused heterocycles have been formed on folic acid molecules; for this reason, and others, our target is to synthesize new derivatives of folic acid as isolated or fused systems. Folic acid 1 reacted with ethyl pyruvate, triethyl orthoformate, ethyl chloroformate, thioformic acid hydrazide, and aldehydes to give new derivatives of folic acid 2-6a,b. Moreover, It reacted with benzylidene malononitrile, acetylacetone, ninhydrin, ethyl acetoacetate, ethyl cyanoacetate, and ethyl chloroacetate to give the pteridine fused systems 10-15, respectively. Ethoxycarbonylamino derivate 5 reacted with some nucleophiles containing the $\mathrm{NH}_{2}$ group, such as aminoguanidinium hydrocarbonate, hydrazine hydrate, glycine, thioformic acid hydrazide, and sulfa drugs in different conditions to give the urea derivatives 16-20a,b. Compound 4 reacted with the same nucleophiles to give the methylidene amino derivatives $\mathbf{2 1 - 2 4 a , b}$. The fused compound $\mathbf{1 0}$ reacted with thioglycolic acid carbon disulfide, malononitrile, and formamide to give the four cyclic fused systems 25-30, respectively. The biological activity of some synthesized showed moderate effect against bacteria, but no effect shown towards fungi.
\end{abstract}

Keywords: folic acid; glutamic acid; imidazo[2,1-b]pteridine; tetrahydroimidazo[2,1- $b$ ]pteridine; biological activity

\section{Introduction}

Folic acid $\mathbf{1}$ is essential to the human metabolic process. It is a key factor in the synthesis of nucleic acid. Folic acid (vitamin $\mathrm{B}_{9}$ ) helps with growth [1] and healthy red blood cells (RBCs) [2]. It is important for cell division. It is essential for growth of the fetus [3]. Folic acid deficiency can lead to human megaloblastic anemia and neural tube defects in fetuses [4], as well as heart diseases, and cancer [5]. To avoid all of these risks, folic acid intake from fortified food has been increasing rapidly [6].

Several novel 2,4-diamino-5-deaza-6,7,8,9-tetrahydropyrido[3,4-g]pteridine derivatives with different substitution at the $\mathrm{N}_{7}$ position were designed and synthesized, as classical and non-classical, partially restricted, linear tricyclic 5-deaza antifolates. The purpose was to investigate the effect of conformational restriction of the $C_{6}-C_{9}(\tau 1)$ and $C_{9}-N_{10}(\tau 2)$ bonds via an ethyl bridge from the $N_{10}$ to the $C_{7}$ position of 5-deaza methotrexate (MTX) on the inhibitory potency against dihydrofolate reductase (DHFR) from different sources and on antitumor activity [7]. Moreover, some efforts were carried out to synthesize the 7-substituted folic acid derivatives the less toxic, more effective, and selective agents for cancer chemotherapy based upon inhibition of dihydrofolate reductase and thymidylate synthetase [8]. The 10-Alkyl-5,10-dideaza analogs of methotrexate and tetrahydrofolic acid were synthesized and used as potent inhibitors of glycineamide ribonucleotide (GAR) formyltransferase [9]. Moreover, numerous fused cyclopenta[d]pyrimidine antifolate were synthesized and examined for their effects as highly potent as DHFR and cell growth inhibitors, and most of them are more potent than methotrexate (MTX) and 10ethyl-10-deazapterin (10-EDAM) in inhibiting tumor cell growth (P388 MTX-sensitive and 
MTX-resistant, colon 26 and KB) on $72 \mathrm{~h}$ drug exposure [10]. Furthermore, 5-deaza-7desmethylene analogues of 5,10-alkylene-5,6,7,8-tetrahydrofolic acid were good substrates for mouse liver folylpolyglutamate synthetase [11].

Bacteria in the intestine synthesizes folic acid [12], which is essential for the progress of the neurological systems of fetuses [13]. Folic acid, as well as the plasma concentration of folate, were inversely associated with hematological, cardiovascular diseases, as well as colorectal cancer [14,15]. Folic acid exhibited anti-inflammatory, antioxidant effects, and decreased levels of interleukins [16,17]. A previous study revealed that folic acid treatment suppressed the inflammatory response of human monocytic cells (THP-1 cells) through the PI3K/Akt pathway [18].

Due to the essential role of folic acid in the synthesis of the bacterial cell wall, we adopted an idea of confusing the bacteria in the synthesis of the cell wall by using a folic acid analogue as an anti-cell wall synthesis drug. Therefore, the target of this work was synthesize some new derivatives from folic acid and screening them against some G+, Gbacteria, and fungi, in the hope of obtaining new antibacterial or antifungal compounds.

The main reaction center of folic acid illustrated in Figure 1.

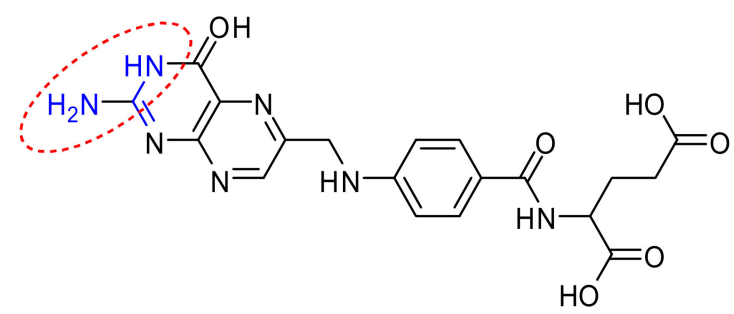

Figure 1. Folic acid reaction center.

\section{Results and Discussion}

The importance of synthesizing a new heterocyclic compound is strongly bonded with the finding of a new drug, capable of destroying any of the diseases that spread around the world. Herein is a new trial, in continuation of our previous work [19-43], to synthesize a new compound from folic acid in the hope of getting a new promoting drug.

Folic acid 1 reacted with ethyl pyruvate in different ratios in DMF as a solvent. Refluxing mixture of folic acid and ethyl pyruvate $1: 1$ ratio for $8 \mathrm{hr}$, gave imidazo[2,1-b]pteridine derivative 2. While the reaction of folic acid with ethyl pyruvate, in a 1:2 ratio for $2 \mathrm{~h}$, led to the formation of the $\mathrm{N}, \mathrm{N}$-disubstituted folic acid (Scheme 1). The structures of the two compounds $\mathbf{2}$ and $\mathbf{3}$ were confirmed from their IR and NMR spectra, where in the IR spectra, the band due to the $\mathrm{NH}_{2}$ group disappeared with appearance of new bands due to the inserted carbonyl groups in the two compounds. While, ${ }^{1} \mathrm{H}-\mathrm{NMR}$ of compound 2 showed singlet signal at $\delta=1.30 \mathrm{ppm}$ due to the $\mathrm{CH}_{3}$ attached to the imidazole ring. The ${ }^{1} \mathrm{H}-\mathrm{NMR}$ of compound 3 also showed more deshielded singlet signal at $\delta=2.21 \mathrm{ppm}$ due to the $2 \mathrm{CH}_{3}$ attached to the carbonyl group. The ${ }^{13} \mathrm{C}-\mathrm{NMR}$ supported the structure proposed where it showed signals due to the $\mathrm{CH}_{3}$ in the two compounds at $\delta=24.51$ and 26.79 ppm, respectively.

Formation of ethoxymethyleneaminopetridine derivative 4 and ethoxycarbonylaminop etridine derivative 5 were formed by the reaction of folic acid with excess triethyl orthoformate and/or ethyl chloroformate in presence of TMA as a base (Scheme 1). The elucidation of the structures of the two compounds were recognized from their spectra and elemental analysis where, the two compounds showed the appearance of new signals in the ${ }^{1} \mathrm{H}-\mathrm{NMR}$, triplets at $\delta=1.15$ and 1.14 quartets at 3.39 and 4.02 ppm due to the $\mathrm{CH}_{3}$ and $\mathrm{CH}_{2}$, respectively. The condensation of the $\mathrm{NH}_{2}$ of folic acid with aldehyde to form Schiff base was carried using formaldehyde and benzaldehyde in acetic acid glacial using drops of $\mathrm{HCl}$ as an acidic catalyst. The Schiff bases formed $\mathbf{6 a}, \mathbf{b}$ showed the disappearance of the $\mathrm{NH}_{2}$ group in folic acid and appearance for the olefinic proton due to the formation of $\mathrm{N}=\mathrm{CH}$ bond at $\delta=7.99$ and $8.03 \mathrm{ppm}$, respectively. ${ }^{13} \mathrm{C}-\mathrm{NMR}$ helped in the structures conforma- 
tion, where, it showed signals at $\delta=139.75$ and $164.21 \mathrm{ppm}$ due to the methylidene and benzylidene carbons respectively. The reaction of thioformic acid hydrazide with folic acid in DMF/EtOH mixture yielded the thiosemicarbazide derivative 7 which cyclized with ethyl chloroformate in presence of TMA to give the 1,3,4-triazole thione derivative 9 (Scheme 1). The structures of compounds 7 and 9 were established from IR and ${ }^{1} \mathrm{HNMR}$, where the IR showed extra bands due to the NH groups in compound 7 and two bands due to $\mathrm{C}=\mathrm{S}$ at 1338 and $1347 \mathrm{~cm}^{-1}$ for compounds 7 and 9 , respectively. Moreover, ${ }^{1} \mathrm{H}-\mathrm{NMR}$ showed three singlet signals for the thiosemicarbazide group at $5.69\left(\mathrm{NH}_{2}\right), 11.31$ and $12.89 \mathrm{ppm}$ for (NHCSNH) protons respectively. Folic acid, also, reacted with acid chloride derivative in basic medium to give the $\mathrm{N}$-substituted derivative. Reaction of folic acid with sebacoyl chloride in presence of TMA yielded the bis compound 8 (Scheme 1). The ${ }^{1} \mathrm{H}$-NMR confirmed the structure of 8 where it showed characteristic signals for the $8 \mathrm{CH}_{2}$ due to the sebacoyl moiety at $\delta=1.03,1.26,2.23$, and $3.34 \mathrm{ppm}$, respectively.<smiles></smiles>

2<smiles>[R]c1cnc2nc(/N=C/OCC)[nH]c(=O)c2n1</smiles>

4<smiles>[R]/C=C/c1nc2ncc([R])nc2c(=O)[nH]1</smiles>

$6 a, b$

$6 a, R^{\prime}=H$

6b, $\mathrm{R}^{\prime}=\mathrm{Ph}$

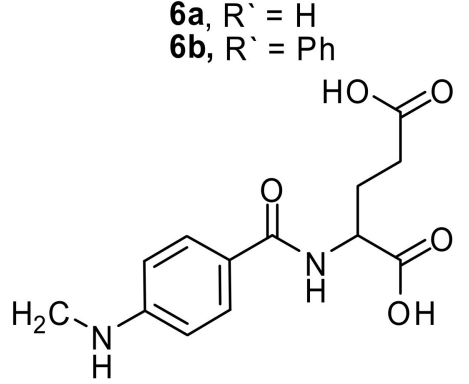<smiles>CCOC(=O)C(C)=O</smiles>

reflux $8 \mathrm{~h}, \mathrm{DMF} /(1: 1)$<smiles>[OH+]=[Co]</smiles><smiles>CC(=O)C(C)=O</smiles>

reflux $2 \mathrm{~h}, \mathrm{DMF} /(1: 2)$<smiles>[R]c1cnc2nc(N(C(C)=O)C(C)=O)[nH]c(=O)c2n1</smiles>
3<smiles>[R]c1cnc2nc(NC(=O)OCC)[nH]c(=O)c2n1</smiles>
5<smiles>[R]OC(=O)CC(C)N(N)C(=S)S</smiles><smiles>[R]c1cnc2nc(NC(=S)NN)[nH]c(=O)c2n1</smiles><smiles>[R]c1cnc2nc(N)[nH]c(=O)c2n1</smiles>

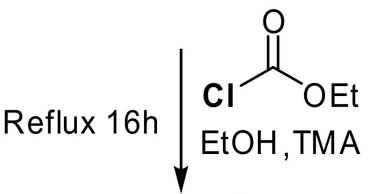<smiles>[R]c1cnc2nc(NC(=O)CC(=O)Nc3nc4ncc([R])nc4c(=O)[nH]3)[nH]c(=O)c2n1</smiles>

8<smiles>[R]c1cnc2nc(-n3c(=O)[nH][nH]c3=S)[nH]c(=O)c2n1</smiles>

Scheme 1. Synthesis some new folic acid derivatives.

Fused systems built in folic acid molecule another target for discovering new promising drug. (Scheme 2), showed some reactions resulted in the formation of fused systems with three or five fused rings. 


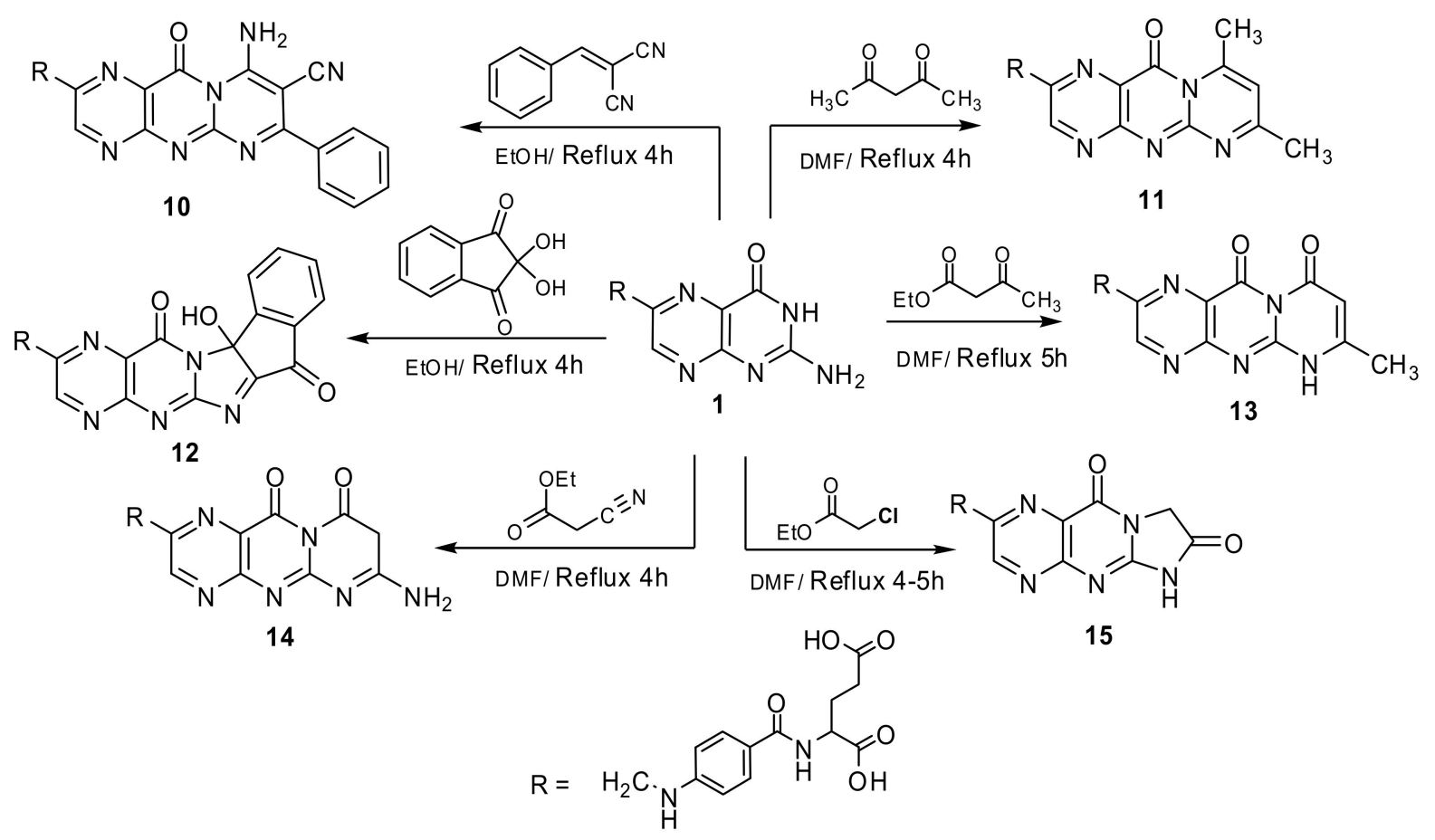

Scheme 2. Synthesis of some new fused systems built on folic acid.

Benzylidene malononitrile was the first compound was used to form the nucleus compound for synthesizing the more fused rings. The well-known benzylidene malononitrile was synthesized and reacted with folic acid in boiling ethanol to form the three fused rings compound pyrimido[2,1-b]pteridine derivative $\mathbf{1 0}$ (Scheme 2) The appearance of the CN band in the IR of compound $\mathbf{1 0}$ was the first guide for the structure assertion. The ${ }^{1} \mathrm{H}-\mathrm{NMR}$ was the second guide, where it showed multiplets at $\delta=7.62-7.96 \mathrm{ppm}$ for the 7-phenyl group. Reaction of folic acid with acetylacetone, ethyl acetoacetate, ethyl cyanoacetate, and / or ethyl chloroacetate in DMF gave fused pyrimidine, fused primidone and fused imidazolidinone to the pteridine ring $\mathbf{1 1}, \mathbf{1 3}, \mathbf{1 4}$, and $\mathbf{1 5}$, respectively (Scheme 2). While the reaction of folic acid with ninhydrin in boiling ethanol yielded the five fused rings derivative indeno[ $\left[2^{\prime}, 1^{\prime}: 4,5\right]$ imidazo[2,1-b]pteridine 12 . The structures of the resulted compounds stablished from their ${ }^{1} \mathrm{H}-\mathrm{NMR}$ and ${ }^{13} \mathrm{C}-\mathrm{NMR}$. The ${ }^{1} \mathrm{H}-\mathrm{NMR}$ of compound $\mathbf{1 1}$ showed two singlets at $\delta=2.26$ and $2.40 \mathrm{ppm}$ for the two $\mathrm{CH}_{3}$ - in pyrimidine ring, Moreover, two signals for the same groups appeared at 24.30 and $26.45 \mathrm{ppm}$ in the ${ }^{13} \mathrm{C}-\mathrm{NMR}$. Compound 13 showed two singlets one for the $\mathrm{CH}_{3}$ at 2.25 and the other at 7.21 due to pyrimidone olefinic proton. The structure of compound $\mathbf{1 4}$ was confirmed by the singlet appeared in its ${ }^{1} \mathrm{H}-\mathrm{NMR}$ at $\delta=2.89 \mathrm{ppm}$ due to pyrimidone $\mathrm{CH}_{2}$, which also appeared in its ${ }^{13} \mathrm{C}-\mathrm{NMR}$ at $\delta=43.05 \mathrm{ppm}$. The disappearance of the $\mathrm{CN}$ band for compound 14 in its IR proved the cyclic structure of the compound. The ${ }^{1} \mathrm{H}-\mathrm{NMR}$ of compound 15 was the main guide for its structure proven, where the ${ }^{1} \mathrm{H}-\mathrm{NMR}$ of compound 15 showed singlet at $\delta=3.51 \mathrm{ppm}$ due to imidazolidinone $\mathrm{CH}_{2}$ along with the presence of $\mathrm{NH}$ singlet signal of imidazolidinone at $\delta=10.87 \mathrm{ppm}$. On the other hand, the structure of compound $\mathbf{1 2}$ was certain, by the presence of the singlet due to the imidazoloindene $\mathrm{OH}$ group at $\delta=5.36 \mathrm{ppm}$.

Ethoxycarbonylamino derivative $\mathbf{1 5}$ was used as starting material for the synthesis of other types of derivatives, which are not attached directly to the pteridine ring. We exploited the chance presence of an ester group in the compound and subjected it to react with some nucleophiles containing $\mathrm{NH}_{2}$, such as aminoguanidinium hydrocarbonate, hydrazine hydrate, glycine, thioformic acid hydrazide, and sulfa drugs in different conditions. Reaction of compound $\mathbf{1 5}$ with aminoguanidinium hydrocarbonate in boiling glacial acetic acid yielded " $N$-\{4-[(\{2-[(\{2-[amino(imino)methyl]hydrazino\}-carbonyl)amino]-4-oxo-3,4dihydropteridin-6-yl\}methyl)amino]benzoyl\}-glutamic acid" 16 (Scheme 3). The structure 
of $\mathbf{1 6}$ was elucidated from the disappearance of the two signals characteristic for the ethyl group in its ${ }^{1} \mathrm{H}-\mathrm{NMR}$, with appearance of multi signals for different $\mathrm{NH}$ present in the compound (experimental part).<smiles>[R]c1cnc2nc(NC(=O)NNC(=N)N)[nH]c(=O)c2n1</smiles><smiles>[R]c1cnc2nc(NC(=O)NN)[nH]c(=O)c2n1</smiles><smiles>N=C(NN)NCC(=O)O</smiles><smiles>[R]c1cnc2nc(NC(=O)OCC)[nH]c(=O)c2n1</smiles><smiles>NCC(=O)OCC(=O)O</smiles><smiles>[R]c1cnc2nc(NC(=O)NCC(=O)O)[nH]c(=O)c2n1</smiles>

18<smiles>[Y]CCCc1ccc(S(=O)(=O)Nc2nc([R])cc([R])n2)cc1</smiles>

Scheme 3. Synthesis of some urea derivatives substituted on folic acid.

Reaction of 15 with $\mathrm{NH}_{2} \mathrm{NH}_{2}$ in boiling DMF yielded the semicarbazide derivative 17. Reaction of 15 with glycine in $\mathrm{DMF} / \mathrm{H} 2 \mathrm{O}$ mixture resulted in the carboxymethylurea derivative 18. Thioformic acid also reacted with compound $\mathbf{1 5}$ in boiling DMSO and gave thiocarboxysemicarbazide derivative 19 (Scheme 3). Conformation of 17, 18, and 19 structures were elucidated form their spectral and analysis data, where all of the ${ }^{1} \mathrm{H}-\mathrm{NMR}$ spectra of all the compounds missing the ethyl group, triplet and quartet signals, with appearance of other signals due to the new functional groups, for example, compound 126, showed in its ${ }^{1} \mathrm{H}-\mathrm{NMR}$ signals at $\delta=4.33,8.69$ and $9.61 \mathrm{ppm}$ for the $\mathrm{NH}_{2}$, and two NH proton neighboring to the carbonyl group. Compound 18 showed the signals corresponding to the glycine molecule, the $\mathrm{CH}_{2}$ protons appeared at $\delta=4.03 \mathrm{ppm}$ and the acidic proton of the glycine carboxylic group found at $11.60 \mathrm{ppm}$. The ${ }^{13} \mathrm{C}-\mathrm{NMR}$ of compound 18 also supported the structure elucidation, where it showed a signal for $\mathrm{CH}_{2}$ carbon at $41.10 \mathrm{ppm}$ and another signal for the glycine carboxylic carbon at $173.17 \mathrm{ppm}$. Compound 19 was the highest data help in the structure confirmation, where, Its IR showed bands due to thiocarboxylic group at $2627 \mathrm{~cm}^{-1}$ for $(\mathrm{SH})$ and $1351 \mathrm{~cm}^{-1}$ for $(\mathrm{C}=\mathrm{S})$ group. Th ${ }^{1} \mathrm{H}-\mathrm{NMR}$ of compound 19 gave more conformational data, it showed signal due to the $\mathrm{SH}$ group at $\delta=13.98 \mathrm{ppm}$, all of this data are supported with the elemental analysis for the sulfur. The presence of the $\mathrm{SH}$ group in compound 19 rejected the idea that compound 19 may cyclize to give 1,3,4-triazole thione derivative.

The combination between the sulfa drug and folic acid was an idea for increasing the biological activity of folic acid, especially antibacterial activity. Thus, folic acid reacted with sulfadiazine and/or sulfadimidine in DMF to yield the corresponding aminocarbonyl sulfadiazine derivative 20a and aminocarbonyl sulfadimidine derivative 20b, respectively (Scheme 3). The structures of $\mathbf{2 0 a}$ and $\mathbf{2 0 b}$ were confirmed from their spectral data (experimental part]. 
Ethoxymethylene derivative $\mathbf{1 4}$ was used for synthesizing a new folic acid derivative; thus, compound 113 reacted with some nucleophilic amino compounds, such as, semicarbazide hydrochloride, aminoguanidine hydrocarbonate, glycine, and sulfa drugs, to obtain some new Schiff base derivatives. Reaction of 4 with semicarbazide hydrochloride in boiling DMF in presence of drops of TMA as a base yielded "N-[4-(\{[2-(\{[2-(aminocarbonyl)hydr azino]methylene\}amino)-4-oxo-3,4-dihydropteridin-6-yl]methyl\}-amino)benzoyl]glutamic acid" 21, while the reaction with aminoguanidine hydrocarbonate in glacial acetic acid yielded the " $N$ - $\{4-[(\{2-[(\{2-[a m i n o(i m i n o) m e t h y l]-h y d r a z i n o\} m e t h y l e n e) a m i n o]-4-o x o-3,4-$ dihydropteridin-6-yl\}methyl)amino]benzoyl\}-glutamic acid" 22. Moreover, reaction of $\mathbf{1 4}$ with glycine in mixture of DMF/ $\mathrm{H} 2 \mathrm{O}(9: 1)$ gave the carboxymethyl)amino derivative 23 (Scheme 4). The structure of the formed compounds 21, 22, and $\mathbf{2 3}$ are confirmed from their IR, ${ }^{1} \mathrm{H}-\mathrm{NMR}$, and ${ }^{13} \mathrm{C}-\mathrm{NMR}$, where all compounds showed the disappearance of the two signals of the ethyl group in their ${ }^{1} \mathrm{H}-\mathrm{NMR}$ with appearance of new signals due to $\mathrm{NH}_{2}$, and $\mathrm{NH}$ groups at the ranges 6.21-6.98 ppm for $\mathrm{NH} 2$ group and 10.13-10.99 ppm for $\mathrm{NH}$ groups. Compound 23 showed in its ${ }^{1} \mathrm{H}-\mathrm{NMR}$ two signals for the glycine moiety, at singlet at $\delta$ $=4.18 \mathrm{ppm}$ for $\mathrm{CH}_{2}$ and at 11.58 for the acidic proton $\mathrm{CH}_{2} \mathrm{COOH}$. Compound 14 reacted with sulfa drugs, namely, sulfadiazine and sulfadimidine, to give the folic acid substituted with sulfa drug moiety, $\mathbf{2 4 a , b}$ (Scheme 4). The structures of compounds $\mathbf{2 4 a}, \mathbf{b}$ proved with the aid of their ${ }^{1} \mathrm{H}-\mathrm{NMR}$, where, the ${ }^{1} \mathrm{H}-\mathrm{NMR}$ showed the appearance of the multiplets due to the phenyl group of the sulfa drug, with disappearance of the ethyl group signals.

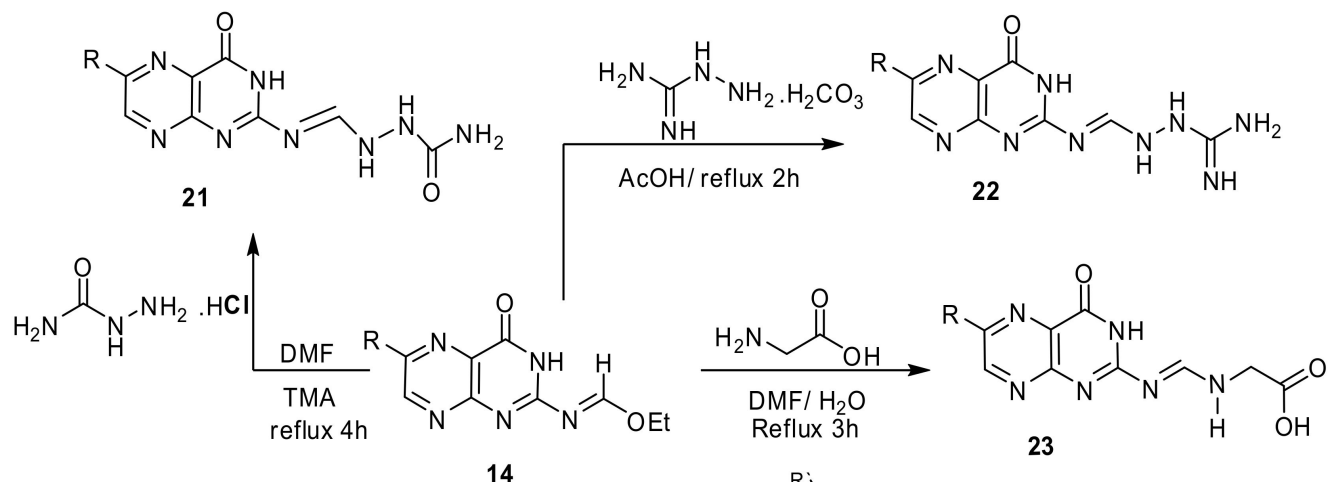<smiles>[R]c1cc([R])nc(NS(=O)(=O)c2ccc(N/C=N/c3nc4ncc([R])nc4c(=O)[nH]3)cc2)n1</smiles>

Scheme 4. Synthesis of some methylene amino derivatives substituted on folic acid.

Finally, compound 10 was used for building some new fused systems on the folic acid molecule. It subjected for some reactions, which gave four ring fused systems as a new form for folic acid derivatives. Compound $\mathbf{1 0}$ reacted with thioglycolic acid in basic conditions to yield the tetracyclic fused system " $N$-(4-\{[(4-amino-3-mercapto-2,12-dioxo-5-phenyl-1,12dihydro-2 $H$-pyrido-[3' $\left.2^{\prime}: 5,6\right]$ pyrimido[2,1-b]pteridin-10-yl)methyl]-amino\}benzoyl)-gluta mic acid" 25 (Scheme 5). Reaction of $\mathbf{1 0}$ with thioglycolic acid boiling ethanol without using TMA yielded the open form mercaptoacetyl derivative $\mathbf{2 6}$ (Scheme 5). The two structures of 25 and 26 in the ${ }^{1} \mathrm{H}-\mathrm{NMR}$ showed the presence of the SH group at 14.00 and $13.97 \mathrm{ppm}$, respectively. The disappearance of the $\mathrm{CN}$ group in the IR of compound 25 confirmed the cyclic form of the compound, while its appearance in the IR of $\mathbf{2 6}$ prove that the structure is an open form. 
<smiles>[R]c1cnc2nc3nc(-c4ccccc4)c4c(N)c(S)c(=O)[nH]c4n3c(=O)c2n1</smiles>

25<smiles>[Z7]c1cnc2nc3nc(-c4ccccc4)c(C#N)c(NC(=S)S)n3c(=O)c2n1</smiles>

27<smiles>[R]c1cnc2nc3nc(-c4ccccc4)c4c(N)c(C#N)c(N)nc4n3c(=O)c2n1</smiles>

29

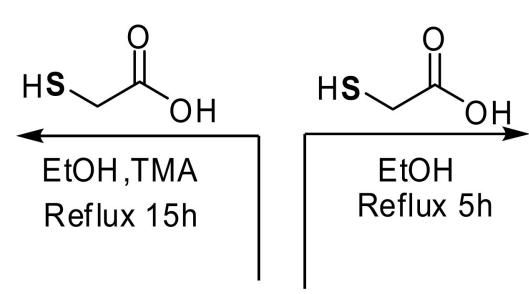<smiles>[R]c1cnc2nc3nc(-c4ccccc4)c(C#N)c(NC(=O)CS)n3c(=O)c2n1</smiles>

26<smiles>[R]c1cnc2nc3nc(-c4ccccc4)c(C#N)c(N)n3c(=O)c2n1</smiles>

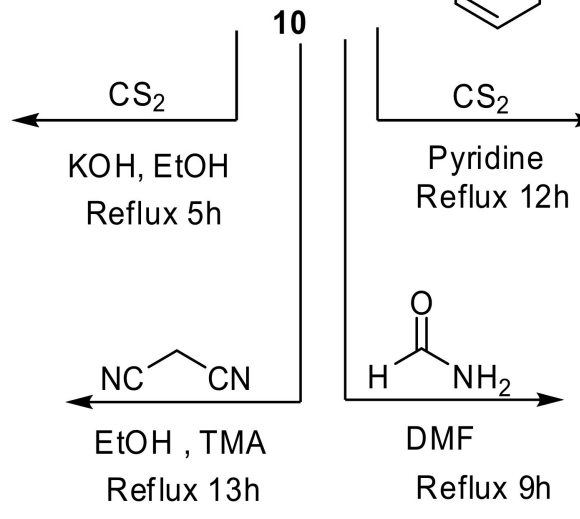<smiles>[R]=CNc1ccc(C(=O)NC(CCC(=O)O)C(=O)O)cc1</smiles><smiles>[R]c1cnc2nc3nc(-c4ccccc4)c4c(=S)[nH]c(=S)[nH]c4n3c(=O)c2n1</smiles><smiles>[R]c1cnc2nc3nc(-c4ccccc4)c4c(N)ncnc4n3c(=O)c2n1</smiles>

30

Scheme 5. Synthesis of some new fused systems on folic acid.

The reaction of carbon sulfide with compound 10 varies with the kind of solvent used, whereas the reaction of $\mathbf{1 0}$ with $\mathrm{CS}_{2}$ in alcoholic $\mathrm{KOH}$ yielded the open form $\mathrm{N}-\{4-[(\{8-\mathrm{cyano}-$ 9-[(mercaptocarbonothioyl)amino]-11-oxo-7-phenyl-11H-pyrimido[2,1-b]pteridin-2-yl\}meth yl)amino]-benzoyl\}glutamic acid 27, while the reaction in boiling dry pyridine yielded the four cyclic ring fused system 28 (Scheme 5). The IR of the two compounds helped in elucidation of the structures, where the IR of compound 27 showed CN band at $2202 \mathrm{~cm}^{-1}$, which are not present in the IR of compound 28; this is an indication that compound 27 is open form and 28 is a cyclic form. Pyrido[ $\left.3^{\prime}, 2^{\prime}: 5,6\right]$ pyrimido[2,1-b]pteridine 29 and pyrimido $\left[5^{\prime}, 4^{\prime}: 5,6\right]$ pyrimido-[2,1-b]pteridine $\mathbf{3 0}$ were prepared by the reaction of $\mathbf{1 0}$ with malononitrile and/or formamide on hot. Compound 29 showed, in its ${ }^{1} \mathrm{H}-\mathrm{NMR}$, two signals for the two $\mathrm{NH}_{2}$ groups at $\delta=6.12$ and $6.58 \mathrm{ppm}$, while compound 30 showed only one signal for the $\mathrm{NH}_{2}$ groups at $\delta=6.50 \mathrm{ppm}$.

\section{Biological Activity}

Antimicrobial activities of some new compounds were tested against some types of $\mathrm{G}^{+}$bacteria, $\mathrm{G}^{-}$bacteria, and fungus, for example, "Bacillus subtilis (ATCC, 6051), Staphylococcus aureus (ATCC, 12600), as a $\mathrm{G}^{+}$bacteria, Escherichia coli (ATCC, 11775), Pseudomonas aeruginosa (ATCC, 10145), as a G- bacteria, Aspergillus flavus link (ATCC, 9643), and Candida 
albicans (ATCC, 7102), as a fungus". Antimicrobial activity of the tested samples was determined using a modified Kirby-Bauer disc diffusion method [44-49].

Biological Activity Results and Discussion

The studying of the biological screening of the tested compounds, Table 1 , showed folic acid had a moderate inhibitory effect against each of the $\mathrm{G}^{+}$and $\mathrm{G}^{-}$bacteria compared to the standard antibacterial agent (Ampicillin).

Table 1. Biological activity of some new synthesized compounds.

\begin{tabular}{|c|c|c|c|c|c|c|}
\hline \multirow{4}{*}{ Sample } & \multicolumn{6}{|c|}{ Inhibition Zone Diameter (mm/mg Sample) } \\
\hline & \multicolumn{4}{|c|}{ Bacterial Species } & \multirow{2}{*}{\multicolumn{2}{|c|}{ Fungi }} \\
\hline & \multicolumn{2}{|c|}{$\left(\mathrm{G}^{+}\right)$} & \multicolumn{2}{|c|}{$\left(\mathrm{G}^{-}\right)$} & & \\
\hline & B. subtilis & S. aureus & E. coli & $\begin{array}{c}P . \\
\text { aeruginosa }\end{array}$ & A. flavus & C. albicans \\
\hline Control: DMSO & 0.0 & 0.0 & 0.0 & 0.0 & 0.0 & 0.0 \\
\hline \multirow{2}{*}{$\begin{array}{c}\text { Ampicillin } \\
\text { Antibacterial agent } \\
\text { Amphotericin B } \\
\text { Antifungal agent }\end{array}$} & 26 & 21 & 25 & 26 & - & - \\
\hline & - & - & - & - & 17 & 21 \\
\hline Folic acid 1 & 10 & 10 & 10 & 10 & 0.0 & 0.0 \\
\hline 2 & 10 & 9 & 10 & 11 & 0.0 & 0.0 \\
\hline 4 & 9 & 9 & 9 & 9 & 0.0 & 0.0 \\
\hline 5 & 0.0 & 0.0 & 0.0 & 0.0 & 0.0 & 0.0 \\
\hline $6 a$ & 12 & 12 & 12 & 13 & 0.0 & 0.0 \\
\hline 8 & 12 & 12 & 13 & 13 & 0.0 & 0.0 \\
\hline 9 & 10 & 0.0 & 0.0 & 12 & 0.0 & 0.0 \\
\hline 10 & 10 & 9 & 9 & 10 & 0.0 & 0.0 \\
\hline 12 & 9 & 10 & 12 & 12 & 0.0 & 0.0 \\
\hline 14 & 10 & 10 & 10 & 10 & 0.0 & 0.0 \\
\hline 15 & 10 & 10 & 11 & 10 & 0.0 & 0.0 \\
\hline 18 & 0.0 & 0.0 & 0.0 & 0.0 & 0.0 & 0.0 \\
\hline $20 \mathrm{~b}$ & 0.0 & 9 & 10 & 0.0 & 0.0 & 0.0 \\
\hline 22 & 13 & 12 & 12 & 12 & 0.0 & 0.0 \\
\hline 23 & 9 & 10 & 10 & 10 & 0.0 & 0.0 \\
\hline $24 b$ & 10 & 9 & 10 & 10 & 0.0 & 0.0 \\
\hline 27 & 11 & 12 & 11 & 14 & 0.0 & 0.0 \\
\hline
\end{tabular}

G: Gram reaction. Solvent: DMSO.

Compounds $\mathbf{6 a}, \mathbf{8}, \mathbf{2 2}, \mathbf{2 6}$, and 27 showed inhibitory effects slightly more than folic acid against all types of bacteria, and this may be due to the presence a new group exceeded to folic acid structure, such as, terminal $\mathrm{C}=\mathrm{N}$ in compounds $\mathbf{6 a}$ and $\mathbf{2 2}, \mathrm{SH}$ in compounds 26 and 27 , and $8 \mathrm{CH}_{2}$ in compound 8 .

Inden-1-one present in compound $\mathbf{1 2}$ may be the cause for more activity than folic acid against $\mathrm{G}^{-}$bacteria E. coli and P. aeruginosa.

Compound 9 as well as compound $20 \mathrm{~b}$ showed somewhat strange inhibitory effects, where compound $\mathbf{9}$ showed higher effect against $P$. aeruginosa than folic acid, and at the same time, it showed null effects against E. coli and S. aureus; this may be due to the presence of triazole thione ring in its structure. Moreover, compound $20 \mathrm{~b}$ showed null 
effects against $B$. subtilis and P. aeruginosa and moderate effect against E. coli and S. aureus, the diamidine nucleus in its structure.

Compounds $2,4,10,14,15,23$, and $24 b$ showed nearly the same effects similar to folic acid; this means that the new groups added to the structure of folic acid did not have any effect against microorganisms in general.

Compounds $\mathbf{5}$ and $\mathbf{1 8}$ had no effect against all microorganisms; this may be due to the presence of each of glycine unit and/or the ethyl carbamate unit in the structure.

In comparison, among the different effects of all compounds under study, we found:

- Compound 22 showed higher effect against B. subtilis than other compounds that showed an average effect.

- Compounds 5, 18, 20b did not show the effect against B. subtilis.

The effect of compounds $\mathbf{6 a}, \mathbf{8}, \mathbf{2 2}, 27$ was slightly higher than 11, 12, 14, 23 against $S$. aureus while other compounds have a moderate effect against $S$. aureus and compounds $\mathbf{1}$, 5, 18 had no effect against them.

The effect of compounds against $E$. coli was close to the effect of compounds against bacteria, while the effect of compounds against $P$. aeruginosa was dissimilar. Compound 27 showed a higher effect against $P$. aeruginosa than other compounds.

On the other hand, folic acid, as well as all of the synthesized compounds, had no effect against Fungi (A. flavus and C. albicans).

\section{Conclusions}

Herein, novel derivatives of folic acid were prepared by direct reactions of different reagents, with folic acid, or by reaction of some derivatives prepared with the same (or another) reagent. The study was directed to find new derivatives of folic acid having a promising biological activity, but, from the study carried out and the results obtained, all of the derivatives prepared were inactive against fungi, while some of these derivatives had a moderate antibacterial activity. The conclusion of this study is that the reactions done on the $\mathrm{NH}_{2}$ group of folic acid, either substituted groups formed or fused systems, are not valuable as antifungal or antibacterial agents. Future work will be directed to the carboxylic groups of folic acid to synthesize a new isolated or fused system from folic acid in hopes of getting a more promising drug.

\subsection{Experimental Chemistry}

All chemicals used were supplied by Sigma (New York, NY, USA). Digital Electro thermal IA 9100 Series used for measuring melting points and they were uncorrected. Infra-red spectra were examined on ATRAlpha FTIR spectrophotometer (Billerica, MA, USA). ${ }^{1} \mathrm{H}-\mathrm{NMR}$ and ${ }^{13} \mathrm{C}-$ NMR spectra examined on a Bruker AC-850 MHz apparatus (Bruker, Billerica, Massachusetts). Chemical shifts expressed as (ppm) relative to internal standard (TMS), and DMSO-d6 used as the solvent and in ${ }^{13} \mathrm{C}-\mathrm{NMR}$ the solvent was $\mathrm{CDCl}_{3}$ and $\mathrm{DMSO}$ mixture. $\mathrm{CHN}$ analyses and biological activity were achieved in Cairo University at Micro-Analytical Center. Spectral data of all compounds are available in Supplementary Materials.

4.1.1. “N-(4-\{[(7-methyl-8,10-dioxo-8,10-dihydroimidazo[2,1-b]pteridin-2yl)methyl]amino\}-benzoyl)glutamic acid" (2)

A mixture of folic acid (0.001 mol, $0.44 \mathrm{~g})$ and ethyl pyruvate $(0.001 \mathrm{~mol}, 0.11 \mathrm{~g})$ dissolved in (15 mL) DMF and refluxed. After $8 \mathrm{hr}$ (TLC, $R_{F}=0.6$, eluent: $\mathrm{CH}_{2} \mathrm{Cl}_{2}$ ) the solvent evaporated under vacuum, the semisolid product formed, poured onto ice, the solid resulted filtered off and crystallized from DMF-ethanol mixture (1:1) to give yellowish brown product. Yield, 87\%, m.p. 223-225 ${ }^{\circ} \mathrm{C}$. IR, 3437, $3407 \mathrm{~cm}^{-1}(2 \mathrm{OH}), 3239-3071 \mathrm{~cm}^{-1}$ (2NH), $2941 \mathrm{~cm}^{-1}$ (Ar-H), $2759 \mathrm{~cm}^{-1}$ (Aliphatic-H), 1689-1599 $\mathrm{cm}^{-1}(5 \mathrm{C}=\mathrm{O}$ and $\mathrm{C}=\mathrm{N})$, $1495 \mathrm{~cm}^{-1}(\mathrm{C}=\mathrm{C}) .{ }^{1} \mathrm{H}-\mathrm{NMR}\left(\mathrm{DMSO} d_{6}, 850 \mathrm{MHz}\right): \delta=1.30\left(\mathrm{~s}, 3 \mathrm{H}, \mathrm{CH}_{3}\right), 1.89-2.01(\mathrm{~m}$, $\left.2 \mathrm{H}, \mathrm{CH}_{2} \mathrm{CH}_{2} \mathrm{COOH}\right), 2.72\left(\mathrm{t}, 2 \mathrm{H}, \mathrm{CH}_{2} \mathrm{CH}_{2} \mathrm{COOH}\right), 4.25(\mathrm{t}, 1 \mathrm{H}, \mathrm{NHC} \underline{\mathrm{HCOOH}}), 4.46$ (s, $2 \mathrm{H}$, pteridine- $\left.\mathrm{CH}_{2}-\mathrm{N}\right), 6.63\left(\mathrm{~d}, 2 \mathrm{H}, \mathrm{N}-\mathrm{Ph}-(\underline{\underline{H}})_{\text {ortho }}\right), 7.24(\mathrm{~s}, 1 \mathrm{H}, \underline{\mathrm{HN}}-\mathrm{Ph}), 7.57$ (d, 2H, N-Ph$\left.(\underline{H})_{\text {meta }}\right), 7.94(\mathrm{~s}, \overline{1 \mathrm{H}}, \mathrm{N} \underline{H C O}), 8.63\left(\mathrm{~s}, 1 \mathrm{H}\right.$, pteridine- $\left.\mathrm{C}_{7} \underline{H}\right), 11.48\left(\mathrm{~s}, 1 \mathrm{H}, \mathrm{CH}_{2} \mathrm{CH}_{2} \mathrm{COO} \underline{H}\right), 12.36$ 
$\left(\mathrm{s}, 1 \mathrm{H}, \mathrm{CH}_{2} \mathrm{COO} \underline{H}\right) .{ }^{13} \mathrm{C}-\mathrm{NMR}\left(\mathrm{DMSO} d_{6}, 200 \mathrm{MHz}\right): \delta=24.51\left(\mathrm{CH}_{3}\right), 30.89\left(\mathrm{CH}_{2} \mathrm{CH}_{2} \mathrm{COOH}\right)$, $33.21\left(\mathrm{CH}_{2} \underline{\mathrm{C}} \mathrm{H}_{2} \mathrm{COOH}\right), 45.95\left(\mathrm{NH}_{\underline{C}} \mathrm{H}_{2}\right), 50.15(\mathrm{NH} \underline{\mathrm{CH}}), 112.42\left(\mathrm{~N}-\mathrm{Ph}-(\underline{\mathrm{C}})_{\text {ortho }}\right), 113.45(\mathrm{~N}-$ $\left.\mathrm{Ph}-(\underline{C})_{\text {Para }}\right), 121.76$ (pteridine $\left.C_{4 a}\right), 128.00\left(\mathrm{~N}-\mathrm{Ph}-(\underline{C})_{\text {meta }}\right), 147.28$ (pteridine $\left.C_{6}\right), 151.73$ (pteridine $C_{7}$ ), $152.77(\mathrm{~N}-\mathrm{Ph}-(\underline{C})), 154.88$ (pteridine $\left.C_{8 a}\right), 157.47$ (pteridine $C_{2}$ ), 162.99 (pteridine $\left.\mathrm{C}_{4}\right), 167.65 \mathrm{NHCO}, 174.25(\mathrm{NHCHCOOH}), 174.87\left(\mathrm{CH}_{2} \mathrm{CH}_{2} \mathrm{COOH}\right)$. Anal. Calcd. for $\mathrm{C}_{22} \mathrm{H}_{19} \mathrm{~N}_{7} \mathrm{O}_{7}$ (493.13): C, 53.55; H, 3.88; N, 19.87; found: $\mathrm{C}, 53.21 ; \mathrm{H}, 3.62 ; \mathrm{N}, 19.77$.

4.1.2. “ $N$-[4-(\{[2-(dipyruvoylamino)-4-oxo-3,4-dihydropteridin-6-yl]methyl\}amino)benzoyl]-glutamic acid" (3)

A mixture of folic acid $(0.001 \mathrm{~mol}, 0.44 \mathrm{~g})$ and ethyl pyruvate $(0.001 \mathrm{~mol}, 0.23 \mathrm{~g})$ in DMF (20 mL) was refluxed for $2 \mathrm{~h}$ until the reaction completed (TLC, $R_{F}=0.5$, eluent: $\mathrm{CH}_{2} \mathrm{Cl}_{2}$ ). The solvent evaporated under vacuum. The precipitate formed, crystallized from EtOH to give yellow product. Yield, 52\%, m.p. $188-190{ }^{\circ} \mathrm{C}$. IR, 3541, $3408 \mathrm{~cm}^{-1}$ (2OH), 3225-3161 cm $\mathrm{cm}^{-1}$ (3NH), 3022-2941 cm $\mathrm{cm}^{-1}$ (Ar-H), $2791 \mathrm{~cm}^{-1}$ (Aliphatic-H), 1684$1599 \mathrm{~cm}^{-1}(8 \mathrm{C}=\mathrm{O}$ and $\mathrm{C}=\mathrm{N}), 1496 \mathrm{~cm}^{-1}(\mathrm{C}=\mathrm{C}) .{ }^{1} \mathrm{HNMR}$ (DMSO $\left.d_{6}, 850 \mathrm{MHz}\right): \delta=1.89-2.01$ $\left(\mathrm{m}, 2 \mathrm{H}, \mathrm{CH}_{2} \mathrm{CH}_{2} \mathrm{COOH}\right), 2.21\left(\mathrm{~s}, 6 \mathrm{H}, 2 \mathrm{CH}_{3}\right), 2.72\left(\mathrm{t}, 2 \mathrm{H}, \mathrm{CH}_{2} \mathrm{CH}_{2} \mathrm{COOH}\right), 4.25(\mathrm{t}, 1 \mathrm{H}$, $\mathrm{NHC} \underline{\mathrm{HCOOH}}), 4.46\left(\mathrm{~s}, 2 \mathrm{H}\right.$, pteridine- $\left.\mathrm{CH}_{2}-\mathrm{N}\right), 6.63\left(\mathrm{~d}, 2 \mathrm{H}, \mathrm{N}-\mathrm{Ph}-(\underline{\hat{H}})_{\text {ortho }}\right), 7.24(\mathrm{~s}, 1 \mathrm{H}, \underline{H \mathrm{~N}}-$ $\mathrm{Ph}), 7.57\left(\mathrm{~d}, 2 \mathrm{H}, \mathrm{N}-\mathrm{Ph}-(\underline{H})_{\text {meta }}\right), 7.94(\mathrm{~s}, 1 \overline{\mathrm{H}}, \mathrm{N} \underline{\mathrm{HCO}}), 8.63\left(\mathrm{~s}, 1 \mathrm{H}\right.$, pteridine- $\left.\mathrm{C}_{7} \underline{H}\right), 10.15(\mathrm{~s}, 1 \mathrm{H}$, pteridine- $\underline{H}$ ), $11.45\left(\mathrm{~s}, 1 \mathrm{H}, \mathrm{CH}_{2} \mathrm{CH}_{2} \mathrm{COO} \underline{H}\right), 12.38\left(\mathrm{~s}, 1 \mathrm{H}, \mathrm{CH}_{2} \mathrm{COOH}\right) .{ }^{13} \mathrm{C}-\mathrm{NMR}$ (DMSO $\left.d_{6}, 200 \mathrm{MHz}\right): \delta=26.79\left(2 \mathrm{CH}_{3}\right), 30.80\left(\underline{\mathrm{CH}}_{2} \mathrm{CH}_{2} \mathrm{COOH}\right), 34.28\left(\mathrm{CH}_{2} \underline{\mathrm{C}} \mathrm{H}_{2} \mathrm{COOH}\right), 45.97$ $\left(\mathrm{NHCH}_{2}\right), 52.35(\mathrm{NHCH}), 111.26\left(\mathrm{~N}-\mathrm{Ph}-(\underline{\mathrm{C}})_{\text {ortho }}\right), 112.56\left(\mathrm{~N}-\mathrm{Ph}-(\underline{\mathrm{C}})_{\text {Para }}\right), 121.61$ (pteridine $\left.C_{4 a}\right), 128.85\left(\mathrm{~N}-\mathrm{Ph}-(\underline{C})_{\text {meta }}\right), 148.38$ (pteridine $\left.C_{6}\right), 150.74$ (pteridine $\left.C_{7}\right), 151.73(\mathrm{~N}-\mathrm{Ph}-(\underline{C}))$, 154.28 (pteridine $\left.C_{8 a}\right), 156.28$ (pteridine $\left.C_{2}\right), 161.39\left(\mathrm{NCOCOCH}_{3}, 162.35\right.$ (pteridine $C_{4}$ ), $\left.166.06 \mathrm{NHCO}, 17 \overline{4.33}(\mathrm{NHCHCOOH}), 174.41\left(\mathrm{CH}_{2} \mathrm{CH}_{2} \mathrm{COOH}\right), 183.35 \mathrm{NCOCOCH}_{3}\right)$. Anal. Calcd. for $\mathrm{C}_{25} \mathrm{H}_{23} \mathrm{~N}_{7} \mathrm{O}_{10}$ (581.49): C, 51.64; $\mathrm{H}, 3.99 ; \mathrm{N}, 16.86$; found: $\mathrm{C}, 51.51 ; \mathrm{H}, 3.82 ; \mathrm{N}, 16.77$.

4.1.3. “N-(4-\{[(2-\{[1-ethoxymethylene]amino\}-4-oxo-3,4-dihydropteridin-6yl)methyl]amino\}-benzoyl)glutamic acid" (4)

Folic acid ( $0.01 \mathrm{~mol}, 4.4 \mathrm{~g}$ ) added to triethyl orthoformate ( $8 \mathrm{~mL}$, excess) and stirred under boiling for $6 \mathrm{~h}$ (TLC, $R_{F}=0.8$, eluent: $\mathrm{CH}_{2} \mathrm{Cl}_{2}$ ). The precipitate formed after cooling filtered and crystallized from EtOH to give yellow-brown powder. Yield, 85\%, m.p. 220 $222{ }^{\circ} \mathrm{C}$. IR, $3470-3400 \mathrm{~cm}^{-1}(2 \mathrm{OH}), 3274-3115 \mathrm{~cm}^{-1}(3 \mathrm{NH}), 2974 \mathrm{~cm}^{-1}(\mathrm{Ar}-\mathrm{H}), 2803 \mathrm{~cm}^{-1}$ (Aliphatic-H), $1693-1601 \mathrm{~cm}^{-1}(4 \mathrm{C}=\mathrm{O}$ and $\mathrm{C}=\mathrm{N}) .{ }^{1} \mathrm{H}-\mathrm{NMR}$ (DMSO $\left.d_{6}, 850 \mathrm{MHz}\right): \delta=1.15$ $\left(\mathrm{t}, 3 \mathrm{H}, \mathrm{CH}_{3}\right), 1.91-2.03\left(\mathrm{~m}, 2 \mathrm{H}, \mathrm{CH}_{2} \mathrm{CH}_{2} \mathrm{COOH}\right), 2.51\left(\mathrm{t}, 2 \mathrm{H}, \mathrm{CH}_{2} \mathrm{CH}_{2} \mathrm{COOH}\right), 3.39(\mathrm{q}, 2 \mathrm{H}$, $\left.\mathrm{CH}_{2}\right), 4.31(\mathrm{t}, 1 \mathrm{H}, \mathrm{NHC} \underline{\mathrm{HCOOH}}), 4.48\left(\mathrm{~s}, 2 \mathrm{H}\right.$, pteridine- $\left.\left.\mathrm{CH}_{2}-\mathrm{N}\right), 6.6 \overline{(\mathrm{d}}, 2 \mathrm{H}, \mathrm{N}-\mathrm{Ph}-(\underline{\mathrm{H}})_{\text {ortho }}\right)$, $6.93(\mathrm{~s}, 1 \mathrm{H}, \underline{H \mathrm{~N}}-\mathrm{Ph}), 7.64\left(\mathrm{~m}, 2 \mathrm{H}, \mathrm{N}-\mathrm{Ph}-(\underline{H}){ }_{(\mathrm{meta})}\right), 7.65(\mathrm{~s}, 1 \mathrm{H}, \mathrm{N}=\mathrm{C} \underline{H}), 8.12(\mathrm{~s}, 1 \mathrm{H}, \mathrm{N} \underline{H C O})$, $8.79\left(\mathrm{~s}, 1 \mathrm{H}\right.$, pteridine- $\left.\mathrm{C}_{7} \underline{\mathrm{H}}\right), 10.38(\mathrm{~s}, 1 \mathrm{H}$, pteridine $\mathrm{NH}), 11.42\left(\mathrm{~s}, 1 \mathrm{H}, \mathrm{CH}_{2} \mathrm{CH}_{2} \mathrm{COO} \underline{H}\right), 12.40$ (s,1H, $\mathrm{CH}_{2} \mathrm{COO} \underline{H}$ ). Anal. Calcd. for $\mathrm{C}_{22} \mathrm{H}_{23} \mathrm{~N}_{7} \mathrm{O}_{7}$ (497.46): C, 53.12; $\mathrm{H}, 4.66 ; \mathrm{N}, 19.71$; found: $\mathrm{C}, 52.89 ; \mathrm{H}, 4.47 ; \mathrm{N}, 19.54$.

4.1.4. “N-\{4-[(\{2-[(ethoxycarbonyl)amino]-4-oxo-3,4-dihydropteridin-6-yl\}methyl)amino]benzoyl\}glutamic acid" (5)

Folic acid $(0.001 \mathrm{~mol}, 0.44 \mathrm{~g})$, ethyl chloroformate $(0.001 \mathrm{~mol}, 0.11 \mathrm{~g})$ and drops of TMA were mixed together in EtOH $(12 \mathrm{~mL})$ and refluxed for $1 \mathrm{~h}$ until the reaction completed (TLC, $R_{F}=0.65$, eluent: $\mathrm{CH}_{2} \mathrm{Cl}_{2}$ ). The solvent evaporated and the precipitate formed crystallized from EtOH to give yellow product. Yield, $87 \%$, m.p. $218-220^{\circ} \mathrm{C}$. IR, $3438,3422 \mathrm{~cm}^{-1}(2 \mathrm{OH}), 3251-3108 \mathrm{~cm}^{-1}(4 \mathrm{NH}), 3079-2939 \mathrm{~cm}^{-1}(\mathrm{Ar}-\mathrm{H}), 2782-2724 \mathrm{~cm}^{-1}$ (Aliphatic-H), $1690-1598 \mathrm{~cm}^{-1}(5 \mathrm{C}=\mathrm{O}$ and $\mathrm{C}=\mathrm{N}), 1496 \mathrm{~cm}^{-1}(\mathrm{C}=\mathrm{C}) .{ }^{1} \mathrm{H}-\mathrm{NMR}$ (DMSO $\left.d_{6}, 850 \mathrm{MHz}\right): \delta=1.14\left(\mathrm{t}, 3 \mathrm{H}, \mathrm{CH}_{2} \mathrm{CH}_{3}\right), 1.90-2.05\left(\mathrm{~m}, 2 \mathrm{H}, \mathrm{CH}_{2} \mathrm{CH}_{2} \mathrm{COOH}\right), 2.31(\mathrm{t}, 2 \mathrm{H}$, $\left.\mathrm{CH}_{2} \mathrm{CH}_{2} \mathrm{COOH}\right), 4.02\left(\mathrm{q}, 2 \mathrm{H}, \mathrm{CH}_{2} \mathrm{CH}_{3}\right), 4.08(\mathrm{t}, 1 \mathrm{H}, \mathrm{NHC} \underline{\mathrm{HOOH}}), 4.53(\mathrm{~s}, 2 \mathrm{H}$, pteridine$\left.\mathrm{CH}_{2}-\mathrm{N}\right), 6.64\left(\mathrm{~d}, 2 \mathrm{H}, \mathrm{N}-\mathrm{Ph}-(\underline{H})_{\text {ortho }}\right), 7.64(\mathrm{~s}, 1 \mathrm{H}, \underline{\mathrm{HN}}-\mathrm{Ph}), 7.65\left(\mathrm{~d}, 2 \mathrm{H}, \mathrm{N}-\mathrm{Ph}-(\underline{H})_{\text {meta }}\right), 8.15$ $(\mathrm{s}, 1 \mathrm{H}, \mathrm{N} \underline{H C O}), 8.21\left(\mathrm{~s}, 1 \mathrm{H}\right.$, pteridine- $\left.\mathrm{C}_{7} \underline{H}\right), 8.72(\mathrm{~s}, 1 \mathrm{H}, \mathrm{N} \underline{H C O O E t}), 10.31(\mathrm{~s}, 1 \mathrm{H}$, pteridine $\mathrm{NH}$ ), $11.51\left(\mathrm{~s}, 1 \mathrm{H}, \mathrm{CH}_{2} \mathrm{CH}_{2} \mathrm{COO} \underline{H}\right), 12.40\left(\mathrm{~s}, 1 \mathrm{H}, \mathrm{CH}_{2} \mathrm{COO} \underline{H}\right) .{ }^{13} \mathrm{C}-\mathrm{NMR}$ (DMSO $d_{6}$, $200 \mathrm{MHz}): \delta=25.09\left(\underline{\mathrm{CH}}_{3} \mathrm{CH}_{2}\right), 30.87\left(\underline{\mathrm{CH}}_{2} \mathrm{CH}_{2} \mathrm{COOH}\right), 34.11\left(\mathrm{CH}_{2} \mathrm{CH}_{2} \mathrm{COOH}\right), 41.20$ 
$\left(\mathrm{CH}_{3} \underline{\mathrm{CH}}_{2}\right), 45.23\left(\mathrm{NH}^{-} \mathrm{H}_{2}\right), 52.10(\mathrm{NH} \underline{\mathrm{CH}}), 111.44$ (N-Ph- $\left.(\underline{\mathrm{C}})_{\text {ortho }}\right), 112.56$ (N-Ph- $\left.(\underline{C})_{\text {Para }}\right)$, 121.61 (pteridine $\left.C_{4 a}\right), 128.85\left(\mathrm{~N}-\mathrm{Ph}-(\underline{C})_{\text {meta }}\right), 148.52$ (pteridine $\left.C_{6}\right), 150.70$ (pteridine $C_{7}$ ), $151.74(\mathrm{~N}-\mathrm{Ph}-(\underline{\mathrm{C}})), 154.27$ (pteridine $\left.C_{8 a}\right), 156.41$ (pteridine $\left.C_{2}\right), 161.89\left(\mathrm{NCOOCH}_{2} \mathrm{CH}_{3}\right)$, 162.35 (pteridine $\left.\mathrm{C}_{4}\right), 166.06(\mathrm{NHCO}), 174.33(\mathrm{NHCH} \underline{\mathrm{COOH}}), 174.41\left(\mathrm{CH}_{2} \mathrm{CH}_{2} \mathrm{COOH}\right)$. Anal. Calcd. for $\mathrm{C}_{22} \mathrm{H}_{23} \mathrm{~N}_{7} \mathrm{O}_{8}$ (513.46): $\mathrm{C}, 51.46 ; \mathrm{H}, 4.51 ; \mathrm{N}, 19.10$; found: $\mathrm{C}, 51.25 ; \mathrm{H}, 4.35 ; \mathrm{N}, 18.84$.

\subsection{Synthesis of Derivatives $\mathbf{6 a}, \mathbf{b}$ (General Procedure)}

Folic acid $(0.001 \mathrm{~mol}, 0.4 \mathrm{~g})$ and appropriate aldehyde $(0.001 \mathrm{~mol})$ mixed in glacial acetic acid $(10 \mathrm{~mL})$ and drops of hydrochloric acid $(0.4 \mathrm{~mL})$ were added, then, the mixture refluxed for $4 \mathrm{~h}$ (TLC, $R_{F}=0.5-0.6$, eluent: $\mathrm{CH}_{2} \mathrm{Cl}_{2}$ ). The formed precipitate filtered and crystallized from EtOH.

4.2.1. “ $N$-[4-(\{[2-(methyleneamino)-4-oxo-3,4-dihydropteridin-6-yl]methyl\}amino)benzoyl]glutamic acid" (6a)

Green powder. Yield, $81 \%$, m.p. $210-212{ }^{\circ} \mathrm{C}$. IR, 3418, $3403 \mathrm{~cm}^{-1}(2 \mathrm{OH}), 3284-$ $3179 \mathrm{~cm}^{-1}$ (3NH), $2934 \mathrm{~cm}^{-1}$ (Ar-H), $2832 \mathrm{~cm}^{-1}$ (Aliphatic-H), $1690-1618 \mathrm{~cm}^{-1}$ (4C=O and $\mathrm{C}=\mathrm{N}), 1590 \mathrm{~cm}^{-1}(\mathrm{C}=\mathrm{C}) .{ }^{1} \mathrm{H}-\mathrm{NMR}\left(\mathrm{DMSO} d_{6}, 850 \mathrm{MHz}\right): \delta=1.88-2.07(\mathrm{~m}, 2 \mathrm{H}$, $\left.\mathrm{CH}_{2} \mathrm{CH}_{2} \mathrm{COOH}\right), 2.71\left(\mathrm{t}, 2 \mathrm{H}, \mathrm{CH}_{2} \mathrm{CH}_{2} \mathrm{COOH}\right), 4.27(\mathrm{t}, 1 \mathrm{H}, \mathrm{NHC} \underline{\mathrm{HCOOH}}), 4.48(\mathrm{~s}, 2 \mathrm{H}$, pteridine- $\left.\mathrm{CH}_{2}-\mathrm{N}\right), 6.59\left(\mathrm{~d}, 2 \mathrm{H}, \mathrm{N}-\mathrm{Ph}-(\underline{\mathrm{H}})_{\text {ortho }}\right), 7.23(\mathrm{~s}, 1 \mathrm{H}, \underline{\mathrm{HN}}-\mathrm{Ph}), 7.55(\mathrm{~m}, 2 \mathrm{H}, \mathrm{N}-\mathrm{Ph}-(\underline{\mathrm{H}})$

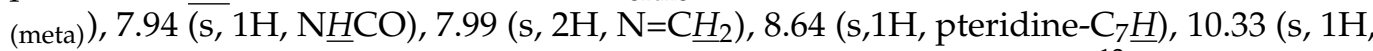
pteridine $\mathrm{NH}), 11.37\left(\mathrm{~s}, 1 \mathrm{H}, \mathrm{CH}_{2} \mathrm{CH}_{2} \mathrm{COO} \underline{H}\right), 12.41\left(\mathrm{~s}, 1 \mathrm{H}, \mathrm{CH}_{2} \mathrm{COOH}\right) .{ }^{13} \mathrm{C}-\mathrm{NMR}$ (DMSO $\left.d_{6}, 200 \mathrm{MHz}\right): \delta=28.15\left(\underline{\mathrm{CH}}_{2} \mathrm{CH}_{2} \mathrm{COOH}\right), 33.15\left(\mathrm{CH}_{2} \mathrm{CH}_{2} \mathrm{COOH}\right), 43.89\left(\mathrm{NH}^{2} \mathrm{H}_{2}\right), 54.56$ $(\mathrm{NHCH}), 112.25\left(\mathrm{~N}-\mathrm{Ph}-(\underline{\mathrm{C}})_{\text {ortho }}\right), 114.01\left(\mathrm{~N}-\mathrm{Ph}-(\underline{\mathrm{C}})_{\mathrm{Para}}\right), 122.47$ (pteridine $\left.\underline{C}_{4 a}\right), 128.25(\mathrm{~N}-\mathrm{Ph}-$ (C) meta), $139.75\left(\mathrm{~N}=\mathrm{CH}_{2}\right), 146.78$ (pteridine $\left.C_{6}\right), 151.20$ (pteridine $\left.C_{7}\right), 152.41(\mathrm{~N}-\mathrm{Ph}-(\underline{C}))$, 154.44 (pteridine $C_{8 a}$ ), 157.61 (pteridine $C_{2}$ ), 163.36 (pteridine $\underline{C O}$ ), $\overline{166.49}$ (NHCO), 175.21 (NHCHCOOH), $175.15\left(\mathrm{CH}_{2} \mathrm{CH}_{2} \mathrm{COOH}\right)$. Anal. Calcd. for $\mathrm{C}_{20} \mathrm{H}_{19} \mathrm{~N}_{7} \mathrm{O}_{6}$ (453.41): C, 52.98; $\mathrm{H}, 4.22 ; \mathrm{N}, 21.62$; found: $\mathrm{C}, 52.71 ; \mathrm{H}, 4.01 ; \mathrm{N}, 21.41$.

\subsection{2. "N-[4-((2-(Benzylideneamino)-4-oxo-3,4-dihydropteridin-6-yl)methyl)-} amino)benzoyl)-glutamic acid" (6b)

Yellow powder. Yield, $62 \%$, m.p. $224-226{ }^{\circ} \mathrm{C}$. IR, 3412, $3399 \mathrm{~cm}^{-1}(2 \mathrm{OH}), 3279$ $3176 \mathrm{~cm}^{-1}$ (3NH), $2951 \mathrm{~cm}^{-1}$ (Ar-H), $2850 \mathrm{~cm}^{-1}$ (Aliphatic-H), $1687-1610 \mathrm{~cm}^{-1}(4 \mathrm{C}=\mathrm{O}$ and $\mathrm{C}=\mathrm{N}), 1550 \mathrm{~cm}^{-1}(\mathrm{C}=\mathrm{C}) .{ }^{1} \mathrm{H}-\mathrm{NMR}\left(\mathrm{DMSO} d_{6}, 850 \mathrm{MHz}\right): \delta=1.85-2.04(\mathrm{~m}, 2 \mathrm{H}$, $\left.\mathrm{CH}_{2} \mathrm{CH}_{2} \mathrm{COOH}\right), 2.73\left(\mathrm{t}, 2 \mathrm{H}, \mathrm{CH}_{2} \mathrm{CH}_{2} \mathrm{COOH}\right), 4.22(\mathrm{t}, 1 \mathrm{H}, \mathrm{NHC} \underline{\mathrm{HCOOH}}), 4.45(\mathrm{~s}, 2 \mathrm{H}$, pteridine- $\left.\mathrm{CH}_{2}-\mathrm{N}\right), 6.61\left(\mathrm{~d}, 2 \mathrm{H}, \mathrm{N}-\mathrm{Ph}-(\underline{\mathrm{H}})_{\text {ortho }}\right), 7.22(\mathrm{~s}, 1 \mathrm{H}, \underline{\mathrm{HN}}-\mathrm{Ph}), 7.57(\mathrm{~m}, 2 \mathrm{H}, \mathrm{N}-\mathrm{Ph}-$ $\left.(\underline{H})_{\text {meta }}\right), 7 . \overline{63}\left(\mathrm{~d}, 1 \mathrm{H}\right.$, benzylidene Ph- $\left.(\underline{H})_{\text {para }}\right), 7.86\left(\mathrm{~d}, 2 \mathrm{H}\right.$, benzylidene Ph- $\left.(\underline{H})_{\text {ortho }}\right), 7.90$ $\left(\mathrm{m}, 2 \mathrm{H}\right.$, benzylidene $\left.\mathrm{Ph}-(H)_{\text {meta }}\right), 7.93(\mathrm{~s}, 1 \mathrm{H}, \mathrm{N} \underline{H C O}), 8.03(\mathrm{~s}, 1 \mathrm{H}$, benzylidene $\mathrm{N}=\mathrm{C}-\mathrm{H})$, $8.60\left(\mathrm{~s}, 1 \mathrm{H}\right.$, pteridine- $\left.\mathrm{C}_{7} \underline{\mathrm{H}}\right), 10.24(\mathrm{~s}, 1 \mathrm{H}$, pteridine $\mathrm{NH}), 11.38\left(\mathrm{~s}, 1 \mathrm{H}, \mathrm{CH}_{2} \mathrm{CH}_{2} \mathrm{COO} \underline{H}\right)$, $12.30\left(\mathrm{~s}, 1 \mathrm{H}, \mathrm{CH}_{2} \mathrm{COO} \underline{H}\right) .{ }^{13} \mathrm{C}-\mathrm{NMR}$ (DMSO $\left.d_{6}, 200 \mathrm{MHz}\right): \delta=30.75\left(\mathrm{CH}_{2} \mathrm{CH}_{2} \mathrm{COOH}\right)$, $33.10\left(\mathrm{CH}_{2} \underline{\mathrm{CH}_{2}} \mathrm{COOH}\right), 45.94\left(\mathrm{NH}_{\underline{C}} \mathrm{H}_{2}\right), 50.07(\mathrm{NH} \underline{\mathrm{C}} \mathrm{H}), 113.20\left(\mathrm{~N}-\mathrm{Ph}-(\underline{\mathrm{C}})_{\text {ortho }}\right), 114.23(\mathrm{~N}-$ $\left.\mathrm{Ph}-(\underline{C})_{\text {Para }}\right), 121.36$ (pteridine $\left.C_{4 a}\right), 128.22\left(\mathrm{~N}-\mathrm{Ph}-(\underline{C})_{\text {meta }}\right), 128.09$ (benzylidene $\left.\mathrm{Ph}_{\text {(meta) }}\right)$, 129.25 (benzylidene $\left.\mathrm{Ph}_{(\text {ortho) }}\right), 131.70$ (benzylidene $\left.\mathrm{Ph}_{(\text {para) }}\right), 133.01$ (benzylidene $\mathrm{CH}-\underline{\mathrm{Ph}}$ ), 146.20 (pteridine $C_{6}$ ), 151.52 (pteridine $C_{7}$ ), $152.44\left(\mathrm{~N}-\mathrm{Ph}-(\underline{\mathrm{C}})\right.$ ), 154.40 (pteridine $C_{8 a}$ ), 157.13 (pteridine $\underline{C}_{2}$ ), 163.36 (pteridine $\underline{\mathrm{CO}}$ ), 164.21 (benzylidene $\mathrm{N}=\mathrm{C}-\mathrm{H}$ ), $167.89 \mathrm{NH} \underline{\mathrm{CO}}, 174.25$ $(\mathrm{NHCH} \underline{\mathrm{COOH}}), 174.54\left(\mathrm{CH}_{2} \mathrm{CH}_{2} \underline{\mathrm{COOH}}\right)$. Anal. Calcd. for $\mathrm{C}_{26} \mathrm{H}_{23} \mathrm{~N}_{7} \mathrm{O}_{6}$ (529.50): C, 58.98; $\mathrm{H}, 4.38 ; \mathrm{N}, 18.52$; found: $\mathrm{C}, 58.74 ; \mathrm{H}, 4.22 ; \mathrm{N}, 18.46$.

4.2.3. “ $N$ - $\{4-[(\{2-[($ hydrazinocarbonothioyl)amino]-4-oxo-3,4-dihydropteridin-6yl\}methyl)-amino]benzoyl\}glutamic acid" (7)

Folic acid $(0.001 \mathrm{~mol}, 0.44 \mathrm{~g})$ dissolved with thioformic acid hydrazide $(0.001 \mathrm{~mol}$, $0.1 \mathrm{~g}$ ) in EtOH/DMF (2:1-15 mL) and stirred under reflux for $10 \mathrm{~h}$. (TLC, $R_{F}=0.4$, eluent: $\mathrm{CH}_{2} \mathrm{Cl}_{2}$ ). The precipitate formed filtered and crystallized from $\mathrm{EtOH}$ to give yellow powder. Yield $91 \%$, m.p. over $300{ }^{\circ} \mathrm{C}$. IR, 3450-3342 $\mathrm{cm}^{-1}(2 \mathrm{OH}), 3342-3049 \mathrm{~cm}^{-1}\left(\mathrm{NH}_{2}\right.$ and $\left.5 \mathrm{NH}\right)$, $2940 \mathrm{~cm}^{-1}$ (Ar-H), $2875 \mathrm{~cm}^{-1}$ (Aliphatic-H), $1685-1600 \mathrm{~cm}^{-1}(4 \mathrm{C}=\mathrm{O}$ and $\mathrm{C}=\mathrm{N}), 1338 \mathrm{~cm}^{-1}$ 
$(\mathrm{C}=\mathrm{S}) .{ }^{1} \mathrm{H}-\mathrm{NMR}\left(\mathrm{DMSO} d_{6}, 850 \mathrm{MHz}\right): \delta=1.89-2.01\left(\mathrm{~m}, 2 \mathrm{H}, \mathrm{CH}_{2} \mathrm{CH}_{2} \mathrm{COOH}\right), 2.49(\mathrm{t}, 2 \mathrm{H}$, $\left.\mathrm{CH}_{2} \mathrm{CH}_{2} \mathrm{COOH}\right), 4.09(\mathrm{t}, 1 \mathrm{H}, \mathrm{NHC} \underline{\mathrm{HCOOH}}), 4.44\left(\mathrm{~s}, 2 \mathrm{H}\right.$, pteridine- $\left.\mathrm{CH}_{2}-\mathrm{N}\right), 5.69(\mathrm{~s}, 2 \mathrm{H}$, $\left.\mathrm{NH}_{2}\right), 6.91\left(\mathrm{~d}, 2 \mathrm{H}, \mathrm{N}-\mathrm{Ph}-(\underline{H})_{\text {ortho }}\right), 6.99(\mathrm{~s}, 1 \mathrm{H}, \underline{\mathrm{HN}}-\mathrm{Ph}), 7.60(\mathrm{~m}, 6 \mathrm{H}, \mathrm{N}-\mathrm{Ph}-(\underline{H})$ (meta) $), 8.10$ (s, $1 \mathrm{H}, \mathrm{N} \underline{H C O}), 8.63\left(\mathrm{~s}, 1 \mathrm{H}\right.$, pteridine- $\left.\mathrm{C}_{7} \underline{H}\right), 10.31(\mathrm{~s}, 1 \mathrm{H}$, pteridine $\mathrm{NH}), 11.31\left(\mathrm{~s}, 1 \mathrm{H}, \mathrm{N}^{H} \mathrm{NH}_{2}\right)$, $11.32\left(\mathrm{~s}, 1 \mathrm{H}, \mathrm{CH}_{2} \mathrm{CH}_{2} \mathrm{COO} \underline{H}\right), 12.18\left(\mathrm{~s}, 1 \mathrm{H}, \mathrm{CH}_{2} \mathrm{COO} \underline{H}\right), 12.66$ (s, 1H, $\underline{\mathrm{H}}$-pyrimidine), 12.89 (s, $1 \mathrm{H}, \mathrm{N} \underline{H}-\mathrm{C}=\mathrm{S}$ ). Calcd. for $\mathrm{C}_{20} \mathrm{H}_{21} \mathrm{~N}_{9} \mathrm{O}_{6} \mathrm{~S}$ (515.50): $\mathrm{C}, 46.60 ; \mathrm{H}, 4.11 ; \mathrm{N}, 24.45$; found: $\mathrm{C}$, $46.43 ; \mathrm{H}, 4.01 ; \mathrm{N}, 24.18$.

4.2.4. “ $\mathrm{N}, \mathrm{N}^{\prime}$-bis[6-(\{[4-(N-glutamincarbonyl)phenyl]amino\}-methyl)-4-oxo-3,4dihydropteridin-2-yl]decanediamide" (8)

Compound $1(0.001 \mathrm{~mol}, 0.88 \mathrm{~g})$ and sebacoyl chloride $(0.001 \mathrm{~mol}, 0.24 \mathrm{~g})$ and drops of TMA in DMF $(10 \mathrm{~mL})$ was refluxed for $6 \mathrm{~h}\left(\mathrm{TLC}, \mathrm{R}_{F}=0.6\right.$, eluent: $\mathrm{CH}_{2} \mathrm{Cl}_{2}$ ). The mixture was poured into ice-cold water, the precipitate obtained crystallized from $\mathrm{EtOH}$ to give orange crystals. Yield, $72 \%$, m.p. $184-186^{\circ} \mathrm{C}$. IR, $3432,3415 \mathrm{~cm}^{-1}(2 \mathrm{OH}), 3270-3160 \mathrm{~cm}^{-1}(4 \mathrm{NH})$, $2951 \mathrm{~cm}^{-1}$ (Ar-H), $2850 \mathrm{~cm}^{-1}$ (Aliphatic-H), $1688-1621 \mathrm{~cm}^{-1}(5 \mathrm{C}=\mathrm{O}$ and $\mathrm{C}=\mathrm{N}), 1347 \mathrm{~cm}^{-1}$ (C=S). ${ }^{1} \mathrm{H}-\mathrm{NMR}$ (DMSO $\left.d_{6}, 850 \mathrm{MHz}\right): \delta=1.03\left(\mathrm{~m}, 4 \mathrm{H}, 2 \mathrm{CH}_{2}, \mathrm{CH}_{2} \mathrm{CH}_{2} \mathrm{CH}_{2} \mathrm{CH}_{2} \mathrm{CONH}\right.$ ), $1.26\left(\mathrm{~m}, 4 \mathrm{H}, 2 \mathrm{CH}_{2}, \mathrm{CH}_{2} \mathrm{CH}_{2} \mathrm{CH}_{2} \mathrm{CH}_{2} \mathrm{CONH}\right), 1.90-2.04\left(\mathrm{t}, 2 \mathrm{H}, \mathrm{CH}_{2} \mathrm{CH}_{2} \mathrm{COOH}\right), 2.23(\mathrm{~m}$, $\left.4 \mathrm{H}, 2 \mathrm{CH}_{2}, \mathrm{CH}_{2} \mathrm{CH}_{2} \mathrm{CH}_{2} \mathrm{CH}_{2} \mathrm{CONH}\right), 2.71\left(\mathrm{t}, 2 \mathrm{H}, \mathrm{CH}_{2} \mathrm{CH}_{2} \mathrm{COOH}\right), 3.34\left(\mathrm{t}, 4 \mathrm{H}, 2 \mathrm{CH}_{2}\right.$, $\left.\mathrm{CH}_{2} \mathrm{CH}_{2} \mathrm{CH}_{2} \mathrm{CH}_{2} \mathrm{CONH}\right), 4.24(\mathrm{t}, 1 \mathrm{H}, \mathrm{NHCHCOOH}), 4.51\left(\mathrm{~s}, 2 \mathrm{H}\right.$, pteridine- $\left.\mathrm{CH}_{2}-\mathrm{N}\right), 6.61(\mathrm{~d}$, 2H, N-Ph- $\left.(\underline{H})_{\text {ortho }}\right), 7.21$ (s, 1H, $\left.\underline{H N}-\mathrm{Ph}\right), 7.49$ (m, 2H, N-Ph- $(\underline{H})$ (meta) $), 7.90(\mathrm{~s}, 1 \mathrm{H}, \mathrm{N} \underline{H} \mathrm{CO})$, $8.61\left(\mathrm{~s}, 1 \mathrm{H}\right.$, pteridine- $\left.\mathrm{C}_{7} \underline{\mathrm{H}}\right), 10.37(\mathrm{~s}, 1 \mathrm{H}$, pteridine $\mathrm{NH}), 11.12\left(\mathrm{~s}, 2 \mathrm{H}, 2 \mathrm{NH}, \mathrm{CH}_{2} \mathrm{CH}_{2} \mathrm{CH}_{2} \mathrm{CH}_{2}\right.$ $\mathrm{CON} \underline{H}), 11.43\left(\mathrm{~s}, 1 \mathrm{H}, \mathrm{CH}_{2} \mathrm{CH}_{2} \mathrm{COO} \underline{H}\right), 12.34\left(\mathrm{~s}, 1 \mathrm{H}, \mathrm{CH}_{2} \mathrm{COO} \underline{H}\right)$. Anal. Calcd. for $\mathrm{C}_{48} \mathrm{H}_{52} \mathrm{~N}_{14} \mathrm{O}_{14}$ (1049.01): C, 54.96; H, 5.00; N, 18.69; found: C, 54.78; H, 4.81; N, 18.52.

4.2.5. “N-[4-(\{[4-oxo-2-(3-oxo-5-thioxo-1,2,4-triazolidin-4-yl)-3,4-dihydro-pteridin-6yl]methyl\}amino)benzoyl]glutamic acid" (9)

Compound 7 ( $0.001 \mathrm{~mol}, 0.51 \mathrm{~g})$ was refluxed for $16 \mathrm{~h}$ with ethyl chloroformate $(0.001 \mathrm{~mol}$, $0.11 \mathrm{~g}$ ) and TMA (3 drops) in EtOH ( $15 \mathrm{~mL}$ ) (TLC, $\mathrm{R}_{F}=0.8$, eluent: $\left.\mathrm{CH}_{2} \mathrm{Cl}_{2}\right)$. A yellow powder formed which filtered and crystallized from EtOH. Yield, $78 \%$, m.p. over $300{ }^{\circ} \mathrm{C}$. IR, $3412-3378 \mathrm{~cm}^{-1}(2 \mathrm{OH}), 3331-3149 \mathrm{~cm}^{-1}(5 \mathrm{NH}), 2961 \mathrm{~cm}^{-1}$ (Ar-H), $2867 \mathrm{~cm}^{-1}$ (Aliphatic-H), $1681-1618 \mathrm{~cm}^{-1}(5 \mathrm{C}=\mathrm{O}$ and $\mathrm{C}=\mathrm{N}), 1350 \mathrm{~cm}^{-1}(\mathrm{C}=\mathrm{S}) .{ }^{1} \mathrm{H}-\mathrm{NMR}\left(\mathrm{DMSO} d_{6}, 850 \mathrm{MHz}\right): \delta$ $=1.90-2.03\left(\mathrm{~m}, 2 \mathrm{H}, \mathrm{CH}_{2} \mathrm{CH}_{2} \mathrm{COOH}\right), 2.497\left(\mathrm{t}, 2 \mathrm{H}, \mathrm{CH}_{2} \mathrm{CH}_{2} \mathrm{COOH}\right), 4.04(\mathrm{~N}-\mathrm{C} \underline{\mathrm{HCOOH}})$, $4.48\left(\mathrm{~s}, 2 \mathrm{H}\right.$, pteridine- $\left.\underline{\mathrm{CH}}_{2}-\mathrm{N}\right), 6.931\left(\mathrm{~d}, 2 \mathrm{H}, \mathrm{N}-\mathrm{Ph}-(\underline{H})_{\text {ortho }}\right), \overline{6.95}(\mathrm{~s}, 1 \mathrm{H}, \underline{\mathrm{HN}}-\mathrm{Ph}), 7.63(\mathrm{~m}, 6 \mathrm{H}$, $\left.\mathrm{N}-\mathrm{Ph}-(\underline{H})_{(\text {meta })}\right), 8.12(\overline{\mathrm{s}}, 1 \mathrm{H}, \mathrm{N} \underline{\mathrm{HCO}}), 8.63\left(\mathrm{~s}, 1 \mathrm{H}\right.$, pteridine- $\left.\mathrm{C}_{7} \underline{H}\right), 10.13(\mathrm{~s}, 1 \mathrm{H}$, triazolidine $\mathrm{N} \underline{\mathrm{HC}}=\mathrm{S}), 10.37(\mathrm{~s}, 1 \mathrm{H}$, pteridine $\mathrm{NH}), 11.31\left(\mathrm{~s}, 1 \mathrm{H}, \mathrm{CH}_{2} \mathrm{CH}_{2} \mathrm{COO} \underline{H}\right), 11.60(\mathrm{~s}, 1 \mathrm{H}$, triazolidine $\mathrm{N} \underline{\mathrm{HC}}=\mathrm{O}), 12.21\left(\mathrm{~s}, 1 \mathrm{H}, \mathrm{CH}_{2} \mathrm{COO} \underline{H}\right), 13.01$ (s, $1 \mathrm{H}, \mathrm{N} \underline{H}$-pyrimidine). ${ }^{13} \mathrm{C}-\mathrm{NMR}$ (DMSO $\left.d_{6}, 200 \mathrm{MHz}\right): \delta=29.66\left(\underline{C}_{2} \mathrm{CH}_{2} \mathrm{COOH}\right), 31.23\left(\mathrm{CH}_{2} \underline{\mathrm{CH}}_{2} \mathrm{COOH}\right), 45.18\left(\mathrm{NH}_{\underline{C}} \mathrm{H}_{2}\right), 53.41$ $(\mathrm{NHCH}), 112.25\left(\mathrm{~N}-\mathrm{Ph}-(\underline{\mathrm{C}})_{\text {ortho }}\right), 114.01\left(\mathrm{~N}-\mathrm{Ph}-(\underline{\mathrm{C}})_{\mathrm{Para}}\right), 122.44$ (pteridine $\left.\mathrm{C}_{4 a}\right), 127.90(\mathrm{~N}-\mathrm{Ph}-$ $\left.(\underline{C})_{\text {meta }}\right), 148.02$ (pteridine $C_{6}$ ), 151.48 (pteridine $\left.C_{7}\right), 153.14\left(\mathrm{~N}-\mathrm{Ph}-(\underline{C})\right.$ ), 154.22 (pteridine $C_{8 a}$ ), 156.32 (triazolidine $\mathrm{C}=\mathrm{O}$ ), 161.01 (pteridine $\underline{C}_{2}$ ), 165.90 (pteridine $\underline{\mathrm{CO}}$ ), 166.05 (NHO), 174.45 $(\mathrm{NHCHCOOH}), 174.58\left(\mathrm{CH}_{2} \mathrm{CH}_{2} \mathrm{COOH}\right), 182.37$ (triazolidine $\mathrm{C}=\mathrm{S}$ ). Calcd. for $\mathrm{C}_{21} \mathrm{H}_{19} \mathrm{~N}_{9} \mathrm{O}_{7} \mathrm{~S}$ (541.50): C, 46.58; H, 3.54; N, 23.28; found: C, 46.33; H, 3.40; N, 23.12.

4.2.6. “ $N$-(4-\{[(9-amino-8-cyano-11-oxo-7-phenyl-11H-pyrimido[2,1-b]pteridin-2yl)methyl]-amino\}benzoyl)glutamic acid" (10)

A mixture of folic acid $(0.001 \mathrm{~mol}, 0.44 \mathrm{~g})$, malononitrile $(0.001 \mathrm{~mol}, 0.66 \mathrm{~g})$, benzaldehyde $(0.11 \mathrm{~g}, 1 \mathrm{mmol})$, and drops of TMA in ethanol $(15 \mathrm{~mL})$ was stirred under reflux for $4 \mathrm{~h}$ (TLC, $R_{F}=0.4$, eluent: $\mathrm{CH}_{2} \mathrm{Cl}_{2}$ ). The reaction cooled at rt (room temperature). then, the precipitate formed filtered and crystallized from $\mathrm{EtOH}$ to give orange crystals. Yield $80 \%$, m.p. $228-230^{\circ} \mathrm{C}$. IR, $3420-3400 \mathrm{~cm}^{-1}(2 \mathrm{OH}), 3250-3071 \mathrm{~cm}^{-1}\left(\mathrm{NH}_{2}\right.$ and $\left.2 \mathrm{NH}\right)$, $2950 \mathrm{~cm}^{-1}$ (Ar-H), $2850 \mathrm{~cm}^{-1}$ (Aliphatic-H), $2217 \mathrm{~cm}^{-1}(\mathrm{CN}), 1682-1598 \mathrm{~cm}^{-1}(4 \mathrm{C}=\mathrm{O}$ and $\mathrm{C}=\mathrm{N}$ ). ${ }^{1} \mathrm{H}-\mathrm{NMR}$ (DMSO $\left.d_{6}, 850 \mathrm{MHz}\right): \delta=1.89-2.02\left(\mathrm{~m}, 2 \mathrm{H}, \mathrm{CH}_{2} \mathrm{CH}_{2} \mathrm{COOH}\right), 2.50$ $\left(\mathrm{t}, 2 \mathrm{H}, \mathrm{CH}_{2} \mathrm{CH}_{2} \mathrm{COOH}\right), 4.02(\mathrm{t}, 1 \mathrm{H}, \mathrm{NHC} \underline{\mathrm{HOOH}}), 4.48\left(\mathrm{~s}, 2 \mathrm{H}\right.$, pteridine- $\left.\mathrm{CH}_{2}-\mathrm{N}\right), 6.65$ $\left(\mathrm{s}, 2 \mathrm{H}, \mathrm{NH}_{2}\right), \overline{6.93}\left(\mathrm{~d}, 2 \mathrm{H}, \mathrm{N}-\mathrm{Ph}-(\underline{H})_{\text {ortho }}\right), 6.95(\mathrm{~s}, 1 \mathrm{H}, \underline{H \mathrm{~N}}-\mathrm{Ph}), 7.62-7.96(\mathrm{~m}, 7 \overline{\mathrm{H}}, \mathrm{N}-\mathrm{Ph}-(\underline{H})$ 
(meta) and pyrimidine-4- $P h), 8.12(\mathrm{~s}, 1 \mathrm{H}, \mathrm{N} \underline{H C O}), 8.65\left(\mathrm{~s}, 1 \mathrm{H}\right.$, pteridine- $\left.\mathrm{C}_{7} \underline{\mathrm{H}}\right), 11.45(\mathrm{~s}, 1 \mathrm{H}$, $\left.\mathrm{CH}_{2} \mathrm{CH}_{2} \mathrm{COO} \underline{H}\right), 12.18\left(\mathrm{~s}, 1 \mathrm{H}, \mathrm{CH}_{2} \mathrm{COO} \underline{H}\right)$. Calcd. for $\mathrm{C}_{29} \mathrm{H}_{23} \mathrm{~N}_{9} \mathrm{O}_{6}$ (593.55): C, 58.68; $\mathrm{H}$, 3.91; N, 21.24; found: C, 58.42; H, 3.68; N, 21.01 .

4.2.7. “N-(4-\{[(7,9-dimethyl-11-oxo-11H-pyrimido[2,1-b]pteridin-2-yl)methyl]amino\}benzoyl) glutamic acid" (11)

Folic acid (0.001 mol, $0.44 \mathrm{~g})$ added to acetylacetone $(0.001 \mathrm{~mol}, 0.1 \mathrm{~g})$ in DMF (12 mL) and stirred under reflux for $4 \mathrm{~h}$ (TLC, $R_{F}=0.8$, eluent: $\mathrm{CH}_{2} \mathrm{Cl}_{2}$ ). The precipitate formed crystallized from EtOH to give reddish-brown product. Yield, 93\%, m.p. $252-254{ }^{\circ} \mathrm{C}$. IR, $3421-3403 \mathrm{~cm}^{-1}(2 \mathrm{OH}), 3266-3214 \mathrm{~cm}^{-1}$ (2NH), $2952 \mathrm{~cm}^{-1}$ (Ar-H), $2853 \mathrm{~cm}^{-1}$ (Aliphatic$\mathrm{H}), 1681-1621 \mathrm{~cm}^{-1}(4 \mathrm{C}=\mathrm{O}$ and $\mathrm{C}=\mathrm{N}) .{ }^{1} \mathrm{H}-\mathrm{NMR}\left(\mathrm{DMSO} d_{6}, 850 \mathrm{MHz}\right): \delta=1.91-2.03(\mathrm{~m}$, $\left.2 \mathrm{H}, \mathrm{CH}_{2} \mathrm{CH}_{2} \mathrm{COOH}\right), 2.26\left(\mathrm{~s}, 3 \mathrm{H}, \mathrm{CH}_{3}\right), 2.40\left(\mathrm{~s}, 3 \mathrm{H}, \mathrm{CH}_{3}\right), 2.53\left(\mathrm{t}, 2 \mathrm{H}, \mathrm{CH}_{2} \mathrm{CH}_{2} \mathrm{COOH}\right)$, $4.24(\mathrm{t}, 1 \mathrm{H}, \mathrm{NHC} \underline{\mathrm{HCOOH}}), 4.46\left(\mathrm{~s}, 2 \mathrm{H}\right.$, pteridine- $\left.\mathrm{CH}_{2}-\mathrm{N}\right), 6.53\left(\mathrm{~d}, 2 \mathrm{H}, \mathrm{N}-\mathrm{Ph}-(\underline{\mathrm{H}})_{\text {ortho }}\right), 6.90$ $(\mathrm{s}, 1 \mathrm{H}, \underline{H \mathrm{~N}}-\mathrm{Ph}), 7.27(\mathrm{~s}, 1 \mathrm{H}$, pyrimidine $\mathrm{C} \underline{H}), 7.56 \overline{(\mathrm{m}}, 2 \mathrm{H}, \mathrm{N}-\mathrm{Ph}-(\underline{H})$ (meta) $), 8.01(\mathrm{~s}, 1 \mathrm{H}$, $\mathrm{NHCO}), 8.62\left(\mathrm{~s}, 1 \mathrm{H}\right.$, pteridine- $\left.\mathrm{C}_{7} \underline{H}\right), 11.27\left(\mathrm{~s}, 1 \mathrm{H}, \mathrm{CH}_{2} \mathrm{CH}_{2} \mathrm{COO} \underline{H}\right), 12.06\left(\mathrm{~s}, 1 \mathrm{H}, \mathrm{CH}_{2} \mathrm{COOH}\right)$. ${ }^{13} \mathrm{CNMR}\left(\mathrm{DMSO} d_{6}, 200 \mathrm{MHz}\right): \delta=24.30\left(\mathrm{CH}_{3}\right), 26.45\left(\mathrm{CH}_{3}\right), 29.28\left(\mathrm{CH}_{2} \mathrm{CH}_{2} \mathrm{COOH}\right), 31.11$ $\left(\mathrm{CH}_{2} \mathrm{CH}_{2} \mathrm{COOH}\right), 45.04\left(\mathrm{NHCH}_{2}\right), 48.47$ (pyrimidine $\left.\mathrm{CH}_{2}\right), 52.49(\mathrm{NHCH}), 111.27(\mathrm{~N}-\mathrm{Ph}-$ $\left.(\underline{C})_{\text {ortho }}\right), 112.58\left(\mathrm{~N}-\mathrm{Ph}-(\underline{\mathrm{C}})_{\text {Para }}\right), 120.95$ (pyrimidine $\left.C_{5}\right), 121.68$ (pteridine $\left.C_{4 a}\right), 127.98(\mathrm{~N}-\mathrm{Ph}$ $\left.(\underline{C})_{\text {meta }}\right), 148.36$ (pteridine $\underline{C_{6}}$ ), 150.72 (pteridine $\left.\underline{C}_{7}\right), 151.71(\mathrm{~N}-\mathrm{Ph}-(\underline{\mathrm{C}})$ )), 154.35 (pteridine $\underline{C_{8 a}}$ ), 161.44 (pteridine $\left.C_{2}\right), 165.95$ (pteridine $\underline{C} \overline{\mathrm{O}}$ ), 166.05 (NHCO), 171.79 (pyrimidine $\mathrm{N}=\mathrm{CH}), 174.45(\mathrm{NHCH} \underline{\mathrm{COOH}}), 174.58\left(\mathrm{CH}_{2} \mathrm{CH}_{2} \underline{\mathrm{COOH}}\right)$. Anal. Calcd. for $\mathrm{C}_{24} \mathrm{H}_{23} \mathrm{~N}_{7} \mathrm{O}_{6}$ (505.48): C, 57.03; H, 4.59; N, 19.40; found: C, 56.71; H, 4.26; N, 19.11.

4.2.8. “N-(4-\{[(13a-hydroxy-5,12-dioxo-12,13a-dihydro-5H-indeno-[2' $\left.1^{\prime}: 4,5\right]$ imidazo[2,1b]pteridin-10-yl)methyl]amino\}benzoyl)-glutamic acid" (12)

Folic acid (0.001 mol, $0.44 \mathrm{~g})$ and ninhydrin $(0.001 \mathrm{~mol}, 0.18 \mathrm{~g})$ in $\mathrm{EtOH}(12 \mathrm{~mL})$ were refluxed for $4 \mathrm{~h}$ (TLC, $R_{F}=0.75$, eluent: $\mathrm{CH}_{2} \mathrm{Cl}_{2}$ ). A yellowish orange crystal formed on hot which, filtered and washed with EtOH. Yield $74 \%$, m.p. $260-262{ }^{\circ} \mathrm{C}$. IR, $3448-$ $3343 \mathrm{~cm}^{-1}(3 \mathrm{OH}), 3248-3073 \mathrm{~cm}^{-1}(2 \mathrm{NH}), 2938 \mathrm{~cm}^{-1}$ (Ar-H), $2788 \mathrm{~cm}^{-1}$ (Aliphatic-H), $1682-1599 \mathrm{~cm}^{-1}(5 \mathrm{C}=\mathrm{O}$ and $\mathrm{C}=\mathrm{N}) .{ }^{1} \mathrm{H}-\mathrm{NMR}\left(\mathrm{DMSO} d_{6}, 850 \mathrm{MHz}\right): \delta=1.94-2.04(\mathrm{~m}, 2 \mathrm{H}$, $\left.\mathrm{CH}_{2} \mathrm{CH}_{2} \mathrm{COOH}\right), 2.51\left(\mathrm{t}, 2 \mathrm{H}, \mathrm{CH}_{2} \mathrm{CH}_{2} \mathrm{COOH}\right), 4.04(\mathrm{t}, 1 \mathrm{H}, \mathrm{NHC} \underline{\mathrm{HCOOH}}), 4.47(\mathrm{~s}, 2 \mathrm{H}$, pteridine- $\left.\mathrm{CH}_{2}-\mathrm{N}\right), 5.36$ (s, 1H, imidazoloindene $\left.\mathrm{OH}\right), 6.91\left(\mathrm{~d}, 2 \mathrm{H}, \mathrm{N}-\mathrm{Ph}-(\underline{H})_{\text {ortho }}\right), 6.95$ (s, $1 \mathrm{H}, \underline{H \mathrm{~N}}-\mathrm{Ph}), 7.62-7.96\left(\mathrm{~m}, 6 \mathrm{H}, \mathrm{N}-\mathrm{Ph}-(\underline{H})_{\text {(meta) }}\right.$ and indene-Ph), $8.11(\mathrm{~s}, 1 \mathrm{H}, \mathrm{NHCO}), 8.63$ (s,1H, pteridine- $\left.\mathrm{C}_{7} \underline{\mathrm{H}}\right), 11.37\left(\mathrm{~s}, 1 \mathrm{H}, \mathrm{CH}_{2} \mathrm{CH}_{2} \mathrm{COO} \underline{H}\right), 12.21\left(\mathrm{~s}, 1 \mathrm{H}, \mathrm{CH}_{2} \mathrm{COO} \underline{H}\right)$. Calcd. for $\mathrm{C}_{28} \mathrm{H}_{21} \mathrm{~N}_{7} \mathrm{O}_{8}$ (583.51): C, 57.63; H, 3.63; N, 16.80; found: C, 57.47; H, 3.41; N, 16.53 .

4.2.9. "N-(4-\{[(7-methyl-9,11-dioxo-6,11-dihydro-9H-pyrimido[2,1-b]pteridin-2yl)methyl]-amino\}benzoyl)glutamic acid" (13)

Folic acid (0.001 mol, $0.44 \mathrm{~g})$ and ethyl acetoacetate $(0.001 \mathrm{~mol}, 0.13 \mathrm{~g})$ in DMF (13 mL) stirred under reflux for $5 \mathrm{~h}$ (TLC, $R_{F}=0.75$, eluent: $\mathrm{CH}_{2} \mathrm{Cl}_{2}$ ). The precipitate separated after cooling crystallized from ethanol to give orange crystals. Yield, 81\%, m.p. $246-248{ }^{\circ} \mathrm{C}$. IR, $3418-3412 \mathrm{~cm}^{-1}(2 \mathrm{OH}), 3271-3223 \mathrm{~cm}^{-1}$ (3NH), $2956 \mathrm{~cm}^{-1}$ (Ar-H), $2850 \mathrm{~cm}^{-1}$ (Aliphatic$\mathrm{H}), 1677-1620 \mathrm{~cm}^{-1}(5 \mathrm{C}=\mathrm{O}$ and $\mathrm{C}=\mathrm{N}) .{ }^{1} \mathrm{H}-\mathrm{NMR}\left(\mathrm{DMSO} d_{6}, 850 \mathrm{MHz}\right): \delta=1.90-2.00(\mathrm{~m}, 2 \mathrm{H}$, $\left.\mathrm{CH}_{2} \mathrm{CH}_{2} \mathrm{COOH}\right), 2.25\left(\mathrm{~s}, 3 \mathrm{H}, \mathrm{CH}_{3}\right), 2.55\left(\mathrm{t}, 2 \mathrm{H}, \mathrm{CH}_{2} \mathrm{CH}_{2} \mathrm{COOH}\right), 4.20(\mathrm{t}, 1 \mathrm{H}, \mathrm{N}-\mathrm{C} \underline{\mathrm{HCOOH}})$, $4 . \overline{43}\left(\mathrm{~s}, 2 \mathrm{H}\right.$, pteridine- $\left.\underline{\mathrm{H}}_{2}-\mathrm{N}\right), 6.51\left(\mathrm{~d}, 2 \mathrm{H}, \mathrm{N}-\mathrm{Ph}-(\underline{H})_{\text {ortho }}\right), 6.93(\mathrm{~s}, 1 \mathrm{H}, \underline{\mathrm{HN}}-\mathrm{Ph}), 7.21(\mathrm{~s}, 1 \mathrm{H}$, pyrimidine $\mathrm{C} \underline{H}), 7.58\left(\mathrm{~m}, \underline{2 \mathrm{H}}, \mathrm{N}-\mathrm{Ph}-(\underline{H})_{(\text {meta) }}\right), 8.04(\mathrm{~s}, 1 \mathrm{H}, \mathrm{N} \underline{H C O}), 8.66\left(\mathrm{~s}, 1 \mathrm{H}\right.$, pteridine- $\left.\mathrm{C}_{7} \underline{H}\right)$, 10.95 (s, $1 \mathrm{H}$, pyrimidine $\mathrm{N} \underline{H}), 11.24\left(\mathrm{~s}, 1 \mathrm{H}, \mathrm{CH}_{2} \mathrm{CH}_{2} \mathrm{COO} \underline{H}\right), 12.14\left(\mathrm{~s}, 1 \mathrm{H}, \mathrm{CH}_{2} \mathrm{COO} \underline{H}\right)$. Anal. Calcd. for $\mathrm{C}_{23} \mathrm{H}_{21} \mathrm{~N}_{7} \mathrm{O}_{7}$ (507.46): C, 54.44; $\mathrm{H}, 4.17 ; \mathrm{N}, 19.32$; found: $\mathrm{C}, 54.13 ; \mathrm{H}, 4.08 ; \mathrm{N}, 19.01$.

4.2.10. “N-(4-\{[(7-amino-9,11-dioxo-8,11-dihydro-9H-pyrimido[2,1-b]pteridin-2yl)methyl]-amino\}benzoyl)glutamic acid" (14)

Folic acid (0.001 mol, $0.44 \mathrm{~g})$ and ethyl cyanoacetate $(0.001 \mathrm{~mol}, 0.13 \mathrm{~g})$ in DMF (15 mL) stirred at boiling point for $4 \mathrm{~h}$ until the reaction finished (TLC, $R_{F}=0.7$, eluent: $\mathrm{CH}_{2} \mathrm{Cl}_{2}$ ). The product obtained after solvent evaporation crystallized from EtOH-DMF to give 
reddish brown product. Yield, 79\%, m.p. $231-2330{ }^{\circ} \mathrm{C}$. IR, 3424, $3412 \mathrm{~cm}^{-1}(2 \mathrm{OH}), 3278-$ $3189 \mathrm{~cm}^{-1}\left(\mathrm{NH}_{2}\right.$ and $\left.2 \mathrm{NH}\right), 2911 \mathrm{~cm}^{-1}(\mathrm{Ar}-\mathrm{H}), 2843 \mathrm{~cm}^{-1}$ (Aliphatic-H), $1679-1624 \mathrm{~cm}^{-1}$ $(5 \mathrm{C}=\mathrm{O}$ and $\mathrm{C}=\mathrm{N}), 1592 \mathrm{~cm}^{-1}(\mathrm{C}=\mathrm{C}) .{ }^{1} \mathrm{H}-\mathrm{NMR}\left(\mathrm{DMSO} d_{6}, 850 \mathrm{MHz}\right): \delta=1.74-2.10(\mathrm{~m}$, $\left.2 \mathrm{H}, \mathrm{CH}_{2} \mathrm{CH}_{2} \mathrm{COOH}\right), 2.73\left(\mathrm{t}, 2 \mathrm{H}, \mathrm{CH}_{2} \mathrm{CH}_{2} \mathrm{COOH}\right), 2.89\left(\mathrm{~s}, 2 \mathrm{H}\right.$, pyrimidine $\left.\mathrm{CH}_{2}\right), 4.22(\mathrm{t}$, $1 \mathrm{H}, \mathrm{NHC} H \mathrm{HCOH}), 4.51\left(\mathrm{~s}, 2 \mathrm{H}\right.$, pteridine- $\left.\mathrm{CH}_{2}-\mathrm{N}\right), 6.63(\mathrm{~d}, 2 \mathrm{H}, \mathrm{N}-\mathrm{Ph}-(\underline{H})$ ortho $), 7.23(\mathrm{~s}, 1 \mathrm{H}$, $\underline{H} \mathrm{~N}-\mathrm{Ph}), 7.56\left(\mathrm{~m}, 2 \mathrm{H}, \mathrm{N}-\mathrm{Ph}-(\underline{\mathrm{H}})_{(\text {meta) }}\right), 7.98(\mathrm{~s}, 1 \mathrm{H}, \mathrm{N} \underline{\mathrm{HCO}}), 8.06\left(\mathrm{~s}, 2 \mathrm{H}, \mathrm{N} \mathrm{H}_{2}\right), 8.61(\mathrm{~s}, 1 \mathrm{H}$, pteridine- $\left.\mathrm{C}_{7} \underline{\mathrm{H}}\right), 11.46\left(\mathrm{~s}, 1 \mathrm{H}, \mathrm{CH}_{2} \mathrm{CH}_{2} \mathrm{COO} \underline{\mathrm{H}}\right), 12.47\left(\mathrm{~s}, 1 \mathrm{H}, \mathrm{CH}_{2} \mathrm{COO} \underline{\mathrm{H}}\right) .{ }^{13} \mathrm{C}-\mathrm{NMR}$ (DMSO $\left.d_{6}, 200 \mathrm{MHz}\right): \delta=28.10\left(\mathrm{CH}_{2} \mathrm{CH}_{2} \mathrm{COOH}\right), 33.19\left(\mathrm{CH}_{2} \mathrm{CH}_{2} \mathrm{COOH}\right), 43.44\left(\mathrm{NHCH}_{2}\right), 43.05$ (pyrimidine $\left.\mathrm{CH}_{2}\right), 54.50(\mathrm{NH} \underline{\mathrm{C}}), 113.22\left(\mathrm{~N}-\mathrm{Ph}-(\underline{\mathrm{C}})_{\text {ortho }}\right), 114.61\left(\mathrm{~N}-\mathrm{Ph}-(\underline{C})_{\text {Para }}\right), 123.34$ (pteridine $\left.C_{4 a}\right), 128.24\left(\mathrm{~N}-\mathrm{Ph}-(\underline{C})_{\text {meta }}\right), 146.32$ (pteridine $\left.C_{6}\right), 151.22$ (pteridine $\left.C_{7}\right), 152.11(\mathrm{~N}-$ $\mathrm{Ph}-(\underline{C})), 154.79$ (pteridine $\underline{C}_{8 a}$ ), 160.09 (pteridine $\underline{C}_{2}$ ), 165.36 (pteridine $\underline{C O}$ ), 166.22 (NHCO), 171.03 (pyrimidine $\mathrm{N}=\mathrm{CH}), 174.12(\mathrm{NHCH} \underline{\mathrm{COOH}}), 175.00\left(\mathrm{CH}_{2} \mathrm{CH}_{2} \underline{\mathrm{COOH}}\right)$. Anal. Calcd. for $\mathrm{C}_{22} \mathrm{H}_{20} \mathrm{~N}_{8} \mathrm{O}_{7}$ (508.44): C, 51.97; H, 3.96; N, 22.04; found: $\mathrm{C}, 51.79 ; \mathrm{H}, 3.81 ; \mathrm{N}, 21.91$.

4.2.11. “N-(4-\{[(7,10-dioxo-6,7,8,10-tetrahydroimidazo[2,1-b]pteridin-2-yl)methyl]amino\}-benzoyl)glutamic acid" (15)

Ethyl chloroacetate $(0.001 \mathrm{~mol}, 0.12 \mathrm{~g})$ and folic acid $(0.001 \mathrm{~mol}, 0.44 \mathrm{~g})$ in DMF $(11 \mathrm{~mL})$ stirred under reflux for $4.5 \mathrm{~h}$ (TLC, $R_{F}=0.8$, eluent: $\mathrm{CH}_{2} \mathrm{Cl}_{2}$ ). The precipitate separated after cooling crystallized from EtOH to yield orange crystals. Yield, $88 \%$, m.p. $221-224{ }^{\circ} \mathrm{C}$. IR, $3423-3401 \mathrm{~cm}^{-1}(2 \mathrm{OH}), 3251-3204 \mathrm{~cm}^{-1}(3 \mathrm{NH}), 2927 \mathrm{~cm}^{-1}$ (Ar-H), $2854 \mathrm{~cm}^{-1}$ (Aliphatic-H), $1668-1618 \mathrm{~cm}^{-1}(5 \mathrm{C}=\mathrm{O}$ and $\mathrm{C}=\mathrm{N}) .{ }^{1} \mathrm{H}-\mathrm{NMR}$ (DMSO $\left.d_{6}, 850 \mathrm{MHz}\right): \delta=1.93-2.04(\mathrm{~m}, 2 \mathrm{H}$, $\left.\mathrm{CH}_{2} \mathrm{CH}_{2} \mathrm{COOH}\right), 2.55\left(\mathrm{t}, 2 \mathrm{H}, \mathrm{CH}_{2} \mathrm{CH}_{2} \mathrm{COOH}\right), 3.51\left(\mathrm{~s}, 2 \mathrm{H}\right.$, imidazolidinone $\left.\mathrm{C}_{2}\right), 4.26(\mathrm{t}, 1 \mathrm{H}$, $\mathrm{NHC} \underline{H C O O H}), 4.48\left(\mathrm{~s}, 2 \mathrm{H}\right.$, pteridine- $\left.\mathrm{CH}_{2}-\mathrm{N}\right), 6.53\left(\mathrm{~d}, 2 \mathrm{H}, \mathrm{N}-\mathrm{Ph}-(\underline{H})_{\text {ortho }}\right), 6.92(\mathrm{~s}, 1 \mathrm{H}, \underline{\mathrm{HN}}-\mathrm{Ph})$, $7.57\left(\mathrm{~m}, 2 \mathrm{H}, \mathrm{N}-\mathrm{Ph}-(\underline{H}){ }_{(\text {meta })}\right), 8.03(\mathrm{~s}, \overline{1 \mathrm{H}}, \mathrm{N} \underline{H C O}), 8.64\left(\mathrm{~s}, 1 \mathrm{H}\right.$, pteridine- $\left.\mathrm{C}_{7} \underline{H}\right), 10.87(\mathrm{~s}, 1 \mathrm{H}$, imidazole $\mathrm{NH}), 11.28\left(\mathrm{~s}, 1 \mathrm{H}, \mathrm{CH}_{2} \mathrm{CH}_{2} \mathrm{COO} \underline{H}\right), 12.11\left(\mathrm{~s}, 1 \mathrm{H}, \mathrm{CH}_{2} \mathrm{COOH}\right)$. Anal. Calcd. for $\mathrm{C}_{21} \mathrm{H}_{19} \mathrm{~N}_{7} \mathrm{O}_{7}$ (481.42): C, 52.39; H, 3.98; N, 20.37; found: C, 52.00; H, 3.71; N, 20.19.

4.2.12. “N-\{4-[(\{2-[(\{2-[amino(imino)methyl]hydrazino\}-carbonyl)amino]-4-oxo-3,4dihydropteridin-6-yl\}methyl)amino]benzoyl\}-glutamic acid" (16)

Compound $15(0.001 \mathrm{~mol}, 0.51 \mathrm{~g})$ and aminoguanidinium hydrocarbonate $(0.001 \mathrm{~mol}$, $0.14 \mathrm{~g})$ in glacial acetic acid $(15 \mathrm{~mL})$ was stirred under reflux for $3 \mathrm{~h}\left(\mathrm{TLC}, R_{F}=0.6\right.$, eluent: $\mathrm{CH}_{2} \mathrm{Cl}_{2}$ ). A brownish powder formed on hot, the precipitate filtered while hot and washed with ethanol. Yield $87 \%$, m.p. $274-276{ }^{\circ} \mathrm{C}$. IR, $3439-3410 \mathrm{~cm}^{-1}(2 \mathrm{OH}), 3319-3161 \mathrm{~cm}^{-1}$ $\left(\mathrm{NH}_{2}\right.$ and $\left.7 \mathrm{NH}\right), 2954 \mathrm{~cm}^{-1}$ (Ar-H), $2851 \mathrm{~cm}^{-1}$ (Aliphatic-H), $1678-1620 \mathrm{~cm}^{-1}(5 \mathrm{C}=\mathrm{O}$ and $\mathrm{C}=\mathrm{N}$ ). ${ }^{1} \mathrm{H}-\mathrm{NMR}$ (DMSO $d_{6}, 850 \mathrm{MHz}$ ): $\delta=1.93-2.05\left(\mathrm{~m}, 2 \mathrm{H}, \mathrm{CH}_{2} \mathrm{CH}_{2} \mathrm{COOH}\right), 2.55$ $\left(\mathrm{t}, 2 \mathrm{H}, \mathrm{CH}_{2} \mathrm{CH}_{2} \mathrm{COOH}\right), 4.31(\mathrm{t}, 1 \mathrm{H}, \mathrm{NHCHCOOH}), 4.54\left(\mathrm{~s}, 2 \mathrm{H}\right.$, pteridine- $\left.\mathrm{CH}_{2}-\mathrm{N}\right), 6.27(\mathrm{~s}$, $\left.2 \mathrm{H}, \mathrm{NH}_{2}\right), 6 . \overline{63}\left(\mathrm{~d}, 2 \mathrm{H}, \mathrm{N}-\mathrm{Ph}-(\underline{H})_{\text {ortho }}\right), 6.91(\mathrm{~s}, 1 \mathrm{H}, \underline{H \mathrm{~N}}-\mathrm{Ph}), 7.65(\mathrm{~m}, 2 \mathrm{H}, \mathrm{N}-\overline{\mathrm{Ph}}-(\underline{H})$ (meta) $)$, $7.80(\mathrm{~s}, 1 \mathrm{H}, \mathrm{C}=\mathrm{NH}), 8.14(\mathrm{~s}, 1 \mathrm{H}, \mathrm{N} \underline{H C O}), 8.51(\mathrm{~s}, 1 \mathrm{H}$, pteridine-N $\underline{H C O N H}), 8.82(\mathrm{~s}, 1 \mathrm{H}$, pteridine- $\left.\mathrm{C}_{7} \underline{\mathrm{H}}\right), 10.17(\mathrm{~s}, 1 \mathrm{H}, \mathrm{N} \underline{H}-\mathrm{NH}), 10.31(\mathrm{~s}, 1 \mathrm{H}$, pteridine $\mathrm{N} \underline{H}), 10.99(\mathrm{~s}, 1 \mathrm{H}, \mathrm{NH}-\mathrm{N} \underline{H})$, 11.40 (s, $\left.1 \mathrm{H}, \mathrm{CH}_{2} \mathrm{CH}_{2} \mathrm{COO} \underline{H}\right), 12.30\left(\mathrm{~s}, 1 \mathrm{H}, \mathrm{CH}_{2} \mathrm{COO} \underline{H}\right)$. Anal. Calcd. for $\mathrm{C}_{21} \mathrm{H}_{23} \mathrm{~N}_{11} \mathrm{O}_{7}$ (541.48): $\mathrm{C}, 46.58 ; \mathrm{H}, 4.28 ; \mathrm{N}, 28.45$; found: $\mathrm{C}, 46.36 ; \mathrm{H}, 4.09 ; \mathrm{N}, 28.31$.

4.2.13. “ $N$ - $\{4-[(\{2-[($ hydrazinocarbonyl)amino]-4-oxo-3,4-dihydropteridin-6yl\}methyl)amino]-benzoyl glutamic acid" (17)

Compound $15(0.51 \mathrm{~g}, 1 \mathrm{mmol})$ and $\mathrm{NH}_{2} \mathrm{NH}_{2}$ (excess, $\left.3 \mathrm{~mL}\right)$ in DMF $(12 \mathrm{~mL})$ were refluxed for $5 \mathrm{~h}$ (TLC, $R_{F}=0.5$, eluent: $\mathrm{CH}_{2} \mathrm{Cl}_{2}$ ). The solution concentrated under vacuum and poured onto crushed ice, an orange compound resulted, crystallized from DMF:EtOH mixture 1:3 to give yellowish powder. Yield, $65 \%$, m.p. $231-233^{\circ} \mathrm{C}$. IR, $3411,3386 \mathrm{~cm}^{-1}$ (2OH), 3309-3214 $\mathrm{cm}^{-1}$ ( $\mathrm{NH}_{2}$ and $\left.5 \mathrm{NH}\right), 3001 \mathrm{~cm}^{-1}$ (Ar-H), $2876 \mathrm{~cm}^{-1}$ (Aliphatic-H), $1686-1618 \mathrm{~cm}^{-1}(5 \mathrm{C}=\mathrm{O}$ and $\mathrm{C}=\mathrm{N}) .{ }^{1} \mathrm{H}-\mathrm{NMR}\left(\mathrm{DMSO}_{6}, 850 \mathrm{MHz}\right): \delta=1.93-2.02(\mathrm{~m}, 2 \mathrm{H}$, $\left.\mathrm{CH}_{2} \mathrm{CH}_{2} \mathrm{COOH}\right), 2.42\left(\mathrm{t}, 2 \mathrm{H}, \mathrm{CH}_{2} \mathrm{CH}_{2} \mathrm{COOH}\right), 4.09(\mathrm{t}, 1 \mathrm{H}, \mathrm{NHCHCOOH}), 4.33(\mathrm{~s}, 2 \mathrm{H}, \mathrm{s}$, 1 H, NHCONHN $\left.\underline{H}_{2}\right), 4.53\left(\mathrm{~s}, 2 \mathrm{H}\right.$, pteridine- $\left.\mathrm{CH}_{2}-\mathrm{N}\right), 6.61\left(\mathrm{~d}, 2 \mathrm{H}, \mathrm{N}-\mathrm{Ph}-(\underline{\mathrm{H}})_{\text {ortho }}\right), 7.62(\mathrm{~s}, 1 \mathrm{H}$, $\underline{H N}-\mathrm{Ph}), 7.68\left(\mathrm{~d}, 2 \mathrm{H}, \mathrm{N}-\mathrm{Ph}-(\underline{H})_{\text {meta }}\right), 8.11(\mathrm{~s}, \overline{1 \mathrm{H}}, \mathrm{N} \underline{H} \mathrm{CO}), 8.22\left(\mathrm{~s}, 1 \mathrm{H}\right.$, pteridine- $\left.\mathrm{C}_{7} \underline{H}\right), 8.69$ (s, $\left.1 \mathrm{H}, \mathrm{NHCON} \underline{\mathrm{H}} \mathrm{NH}_{2}\right), 9.61\left(\mathrm{~s}, 1 \mathrm{H}, \mathrm{N} \underline{\mathrm{HCONHNH}}{ }_{2}\right), 10.34(\mathrm{~s}, 1 \mathrm{H}$, pteridine $\mathrm{NH}), 11.41$ 
$\left(\mathrm{s}, 1 \mathrm{H}, \mathrm{CH}_{2} \mathrm{CH}_{2} \mathrm{COO} \underline{H}\right), 12.34\left(\mathrm{~s}, 1 \mathrm{H}, \mathrm{CH}_{2} \mathrm{COO} \underline{H}\right) .{ }^{13} \mathrm{C}-\mathrm{NMR}\left(\mathrm{DMSO} d_{6}, 200 \mathrm{MHz}\right): \delta=31.18$ $\left(\underline{\mathrm{C}} \mathrm{H}_{2} \mathrm{CH}_{2} \mathrm{COOH}\right), 35.41\left(\mathrm{CH}_{2} \underline{C H}_{2} \mathrm{COOH}\right), 47.13\left(\mathrm{NHCH}_{2}\right), 51.99(\mathrm{NH} \underline{\mathrm{CH}}), 112.12(\mathrm{~N}-\mathrm{Ph}-$ $\left.(\underline{\mathrm{C}})_{\text {ortho }}\right), 114.43\left(\mathrm{~N}-\mathrm{Ph}-(\underline{\mathrm{C}})_{\text {Para }}\right), 121.67$ (pteridine $\left.\mathrm{C}_{4 a}\right), 129.11$ (N-Ph- $\left.(\underline{\mathrm{C}})_{\text {meta }}\right), 147.71$ (pteridine $\left.C_{6}\right), 151.58$ (pteridine $\left.C_{7}\right), 151.78(\mathrm{~N}-\mathrm{Ph}-(\underline{\mathrm{C}})), 155.41$ (pteridine $\left.C_{8 a}\right), 158.18$ (pteridine $\left.\mathrm{C}_{2}\right), 161.89\left(\mathrm{NCONHNH}_{2}\right), 162.11$ (pteridine $\left.\mathrm{C}_{4}\right), 166.43(\mathrm{NHCO}), 175.81(\mathrm{NHCH} \underline{\mathrm{COOH}})$, $177.71\left(\mathrm{CH}_{2} \mathrm{CH}_{2} \mathrm{COOH}\right)$. Anal. Calcd. for $\mathrm{C}_{20} \overline{\mathrm{H}}_{21} \mathrm{~N}_{9} \mathrm{O}_{7}$ (499.44): C, 48.10; $\mathrm{H}, 4.24 ; \mathrm{N}, 25.24$; found: $\mathrm{C}, 47.72 ; \mathrm{H}, 4.11 ; \mathrm{N}, 25.18$.

4.2.14. “N-[4-(\{[2-(\{[(carboxymethyl)amino]carbonyl $\} a m i n o)-4-o x o-3,4-d i h y d r o p t e r i d i n-6-$ yl]methyl amino)benzoyl]glutamic acid" (18)

Compound 15 (0.001 mol, $0.5 \mathrm{~g})$ and glycine (0.001 mol, $0.08 \mathrm{~g})$ in DMF/ $\mathrm{H}_{2} \mathrm{O}$ mixture $\left(9: 1,10 \mathrm{~mL}\right.$ ) was refluxed for $4 \mathrm{~h}$ (TLC, $R_{F}=0.70$, eluent: $\mathrm{CH}_{2} \mathrm{Cl}_{2}$ ). The solvent concentrated by evaporation. The brown solid formed after pouring on crushed ice crystallized from EtOH. Yield, 83\%, m.p. $210-212{ }^{\circ} \mathrm{C}$. IR, 3451-3410 cm ${ }^{-1}(3 \mathrm{OH}), 3233-3185 \mathrm{~cm}^{-1}(3 \mathrm{NH})$, $2971 \mathrm{~cm}^{-1}$ (Ar-H), $2857 \mathrm{~cm}^{-1}$ (Aliphatic-H), $1674-1623 \mathrm{~cm}^{-1}(6 \mathrm{C}=\mathrm{O}$ and $\mathrm{C}=\mathrm{N}) .{ }^{1} \mathrm{H}-\mathrm{NMR}$ $\left(\mathrm{DMSO} d_{6}, 850 \mathrm{MHz}\right): \delta=1.92-2.01\left(\mathrm{~m}, 2 \mathrm{H}, \mathrm{CH}_{2} \mathrm{CH}_{2} \mathrm{COOH}\right), 2.55\left(\mathrm{t}, 2 \mathrm{H}, \mathrm{CH}_{2} \mathrm{CH}_{2} \mathrm{COOH}\right)$, $4.03\left(\mathrm{~s}, 2 \mathrm{H}, \mathrm{NHCH} \mathrm{H}_{2} \mathrm{COOH}\right), 4.33(\mathrm{t}, 1 \mathrm{H}, \mathrm{NHC} \underline{\mathrm{HCOOH}}), 4.51\left(\mathrm{~s}, 2 \mathrm{H}\right.$, pteridine-C $\left.\overline{\mathrm{H}_{2}}-\mathrm{N}\right), 6.58$ $\left(\mathrm{d}, 2 \mathrm{H}, \mathrm{N}-\mathrm{Ph}-(\underline{H})_{\text {ortho }}\right), 6.96(\mathrm{~s}, 1 \mathrm{H}, \underline{H \mathrm{~N}}-\mathrm{Ph}), 7.61$ (m, 2H, N-Ph- $\left.(\underline{H})_{(\text {meta })}\right), 8.01(\mathrm{~s}, \overline{\mathrm{H}}, \mathrm{N} \underline{H C O})$, $8.66\left(\mathrm{~s}, 1 \mathrm{H}\right.$, pteridine $\left.-\mathrm{C}_{7} \underline{H}\right), 8.78\left(\mathrm{~s}, 1 \mathrm{H}, \mathrm{N} \underline{H} \mathrm{CH}_{2} \mathrm{COOH}\right), 10.31(\mathrm{~s}, 1 \mathrm{H}$, pteridine $\mathrm{N} \underline{H}), 11.31$ (s, $\left.1 \mathrm{H}, \mathrm{CH}_{2} \mathrm{CH}_{2} \mathrm{COO} \underline{H}\right), 11.60\left(\mathrm{~s}, 1 \mathrm{H}, \mathrm{CH}_{2} \mathrm{COO} \underline{H}\right), 12.22\left(\mathrm{~s}, 1 \mathrm{H}, \mathrm{NHCH}_{2} \mathrm{COO} \underline{H}\right) .{ }^{13} \mathrm{CNMR}$ $\left(\mathrm{DMSO} d_{6}, 200 \mathrm{MHz}\right): \delta=25.08\left(\mathrm{CH}_{3}\right), 30.19\left(\mathrm{CH}_{2} \mathrm{CH}_{2} \mathrm{COOH}\right), 34.13\left(\mathrm{CH}_{2} \mathrm{CH}_{2} \mathrm{COOH}\right)$, 41.10 (glycine $\left.\mathrm{CH}_{2}\right), 45.90\left(\mathrm{NH}_{\underline{C}}{ }_{2}\right), 51.21(\mathrm{NH} \underline{\mathrm{CH}}), 111.18$ (N-Ph- $\left.(\underline{\mathrm{C}})_{\text {ortho }}\right), 120.99$ (N-Ph$(\underline{C})$ Para $), 127.97$ (pteridine $\left.C_{4 a}\right), 128.69\left(\mathrm{~N}-\mathrm{Ph}-(\underline{\mathrm{C}})_{\text {meta }}\right), 148.09$ (pteridine $\left.\mathrm{C}_{6}\right), 150.67$ (pteridine $\left.C_{7}\right), 150.91(\mathrm{~N}-\mathrm{Ph}-(\underline{C})), 154.69$ (pteridine $\left.C_{8 a}\right), 156.30$ (pteridine $C_{2}$ ), $\overline{160.91}$ (pteridine $C_{4}$ ), 161.53 (NHCONH), 166.52 NHCO, $172 . \overline{09}$ (NHCHCOOH), $17 \overline{3.17}$ (glycine CO), $17 \overline{4.51}$ $\left(\mathrm{CH}_{2} \mathrm{CH}_{2} \mathrm{COOH}\right)$. Anal. Calcd. for $\mathrm{C}_{22} \mathrm{H}_{22} \mathrm{~N}_{8} \mathrm{O}_{9}$ (542.46): C, 48.71; H, 4.09; N, 20.66; found: C, 48.53; H, 3.78; N, 20.51.

4.2.15. “N-[4-(\{[2-(\{[2-(mercaptocarbonothioyl)hydrazino]-carbonyl $\} a m i n o)-4-o x o-3,4-$ dihydropteridin-6-yl]methyl\}amino)benzoyl]glutamic acid" (19)

Compound 15 (0.001 mol, $0.51 \mathrm{~g})$ and thioformic acid hydrazide $(0.001 \mathrm{~mol}, 0.1 \mathrm{~g})$ in DMSO $(12 \mathrm{~mL})$ were refluxed for $5 \mathrm{~h}\left(\mathrm{TLC}, \mathrm{R}_{F}=0.4\right.$, eluent: $\mathrm{CH}_{2} \mathrm{Cl}_{2}$ ). The product formed after pouring onto crushed ice crystallized from DMF/EtOH 1:1 to yield brown powder. Yield, 84\%, m.p. $281-283{ }^{\circ} \mathrm{C}$. IR, 3423-3402 $\mathrm{cm}^{-1}(2 \mathrm{OH}), 3251-3147 \mathrm{~cm}^{-1}(6 \mathrm{NH}), 2928 \mathrm{~cm}^{-1}$ $\left(\right.$ Ar-H), $2850 \mathrm{~cm}^{-1}$ (Aliphatic-H), $2627 \mathrm{~cm}^{-1}(\mathrm{SH}), 1678-1621 \mathrm{~cm}^{-1}(5 \mathrm{C}=\mathrm{O}$ and $\mathrm{C}=\mathrm{N})$, $1351 \mathrm{~cm}^{-1}(\mathrm{C}=\mathrm{S}) .{ }^{1} \mathrm{H}-\mathrm{NMR}\left(\mathrm{DMSO} d_{6}, 850 \mathrm{MHz}\right): \delta=1.89-2.04\left(\mathrm{~m}, 2 \mathrm{H}, \mathrm{CH}_{2} \mathrm{CH}_{2} \mathrm{COOH}\right)$, $2.50\left(\mathrm{t}, 2 \mathrm{H}, \mathrm{CH}_{2} \mathrm{CH}_{2} \mathrm{COOH}\right), 4.08\left(\mathrm{~s}, 2 \mathrm{H}, \mathrm{NHCH}_{2} \mathrm{COOH}\right), 4.31(\mathrm{t}, 1 \mathrm{H}, \mathrm{NHC} \underline{\mathrm{HCOOH}}), 4.54$ (s, 2H, pteridine- $\left.\overline{\mathrm{CH}}_{2}-\mathrm{N}\right), 6.54\left(\mathrm{~d}, 2 \mathrm{H}, \mathrm{N}-\mathrm{Ph}-(\underline{\mathrm{H}})_{\text {ortho }}\right), 6.96(\mathrm{~s}, 1 \mathrm{H}, \underline{\mathrm{HN}}-\mathrm{Ph}), 7.62(\mathrm{~m}, 2 \mathrm{H}$, $\left.\mathrm{N}-\mathrm{Ph}-(\underline{H})_{(\text {meta) }}\right), 8 . \overline{09}$ (s, $\left.1 \mathrm{H}, \mathrm{N} \underline{H C O}\right), 8.61$ (s,1H, pteridine- $\left.\mathrm{C}_{7} \underline{H}\right), 10.36$ (s, $1 \mathrm{H}$, pteridine

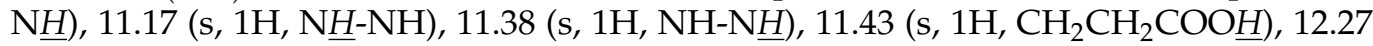
(s,1H, $\mathrm{CH}_{2} \mathrm{COO} \underline{H}$ ), 13.98 (s, $1 \mathrm{H}, \mathrm{S} \underline{\mathrm{H}}$ ). Anal. Calcd. for $\mathrm{C}_{21} \mathrm{H}_{21} \mathrm{~N}_{9} \mathrm{O}_{7} \mathrm{~S}_{2}$ (575.58): C, 43.82; $\mathrm{H}$, 3.68; N, 21.90; S, 11.14; found: C, 43.57; H, 3.49; N, 21.77; S, 10.91.

\subsection{Reaction of Compound 15 with Sulfa Drugs (20a,b): General Procedure}

A mixture of 15 (0.001 mol, $0.51 \mathrm{~g})$ and appropriate sulfa drug (0.001 mol) in DMF (15 mL) was stirred under reflux for $4 \mathrm{~h}$ (TLC, $R_{F}=0.45$, eluent: $\mathrm{CH}_{2} \mathrm{Cl}_{2}$ ). The precipitate formed crystallized from $\mathrm{EtOH}$ to produce yellow to orange powder.

4.3.1. “N-(4-\{[(4-oxo-2-\{[(\{4-[(pyrimidin-2-ylamino)sulfonyl]-phenyl $\}$ amino $)$ carbonyl]amino\}-3,4-dihydropteridin-6-yl)methyl]amino\}-benzoyl)glutamic acid" (20a)

Yellowish orange, yield, $77 \%$, m.p. $238-240{ }^{\circ} \mathrm{C}$. IR, 3438-3417 $\mathrm{cm}^{-1}(2 \mathrm{OH}), 3331-3199$ $\mathrm{cm}^{-1}$ (6NH), $2952 \mathrm{~cm}^{-1}$ (Ar-H), $2853 \mathrm{~cm}^{-1}$ (Aliphatic-H), 1684-1623 $\mathrm{cm}^{-1}$ (5C=O and $\mathrm{C}=\mathrm{N}) .{ }^{1} \mathrm{H}-\mathrm{NMR}\left(\mathrm{DMSO}_{6}, 850 \mathrm{MHz}\right): \delta=1.88-2.04\left(\mathrm{~m}, 2 \mathrm{H}, \mathrm{CH}_{2} \mathrm{CH}_{2} \mathrm{COOH}\right), 2.51(\mathrm{t}, 2 \mathrm{H}$, 
$\left.\mathrm{CH}_{2} \mathrm{CH}_{2} \mathrm{COOH}\right), 4.34(\mathrm{t}, 1 \mathrm{H}, \mathrm{NHC} \underline{\mathrm{HCOOH}}), 4.55\left(\mathrm{~s}, 2 \mathrm{H}\right.$, pteridine- $\left.\mathrm{CH}_{2}-\mathrm{N}\right), 6.42(\mathrm{~d}, 2 \mathrm{H}$, $J=8.4 \overline{\mathrm{Hz}}$, benzene $\left.\mathrm{C}_{2} \underline{H}, \mathrm{C}_{6} \underline{H}\right), 6.65\left(\mathrm{~d}, 2 \mathrm{H}, \mathrm{N}-\mathrm{Ph}-(\underline{H})_{\text {ortho }}\right), 6.81(\mathrm{~d}, 2 \mathrm{H}, \bar{J}=8.4 \mathrm{~Hz}$, benzene $\left.\mathrm{C}_{3} \underline{H}, \mathrm{C}_{5} \underline{H}\right), 6.92(\mathrm{~s}, 1 \mathrm{H}, \underline{H \mathrm{~N}}-\mathrm{Ph}), 7.66\left(\mathrm{~m}, 2 \mathrm{H}, \mathrm{N}-\mathrm{Ph}-(\underline{H})_{(\text {meta) }}\right), 8.17$ (s, 1H, N $\left.\underline{H C O}\right), 8.41$ (t, $1 \mathrm{H}$, pyrimidine $\left.\mathrm{C}_{3} \underline{H}\right), 8.53(\mathrm{~s}, 1 \mathrm{H}$, pteridine- $\mathrm{N} \underline{H C O N H}), 8.64\left(\mathrm{~d}, 2 \mathrm{H}\right.$, pyrimidine $\left.\mathrm{C}_{2} \underline{H}, \mathrm{C}_{4} \underline{H}\right)$, $8.77\left(\mathrm{~s}, 1 \mathrm{H}\right.$, pteridine- $\left.\mathrm{C}_{7} \underline{H}\right), 8.99(\mathrm{~s}, 1 \mathrm{H}$, pteridine-NHCON$\underline{H}), 10.31(\mathrm{~s}, 1 \mathrm{H}$, pteridine $\mathrm{N} \underline{H})$, $11.38\left(\mathrm{~s}, 1 \mathrm{H}, \mathrm{CH}_{2} \mathrm{CH}_{2} \mathrm{COOH}\right), 12.27\left(\mathrm{~s}, 1 \mathrm{H}, \mathrm{CH}_{2} \mathrm{COOH}\right), 12.83\left(\mathrm{~s}, 1 \mathrm{H}, \mathrm{SO}_{2} \mathrm{~N} \underline{H}\right)$. Anal. Calcd. for $\mathrm{C}_{30} \mathrm{H}_{27} \mathrm{~N}_{11} \mathrm{O}_{9} \mathrm{~S}$ (717.67): C, 50.21; H, 3.79; N, 21.47; S, 4.47; found: $\mathrm{C}, 49.91 ; \mathrm{H}, 3.58 ; \mathrm{N}$, $21.62 ; \mathrm{S}, 4.30$.

4.3.2. “ $N$-[4-(\{[2-(\{[(4-\{[(4,6-dimethylpyrimidin-2-yl)amino]-sulfonyl $\}$ phenyl)amino]carbonyl\}amino)-4-oxo-3,4-dihydropteridin-6-yl]methyl\}amino) benzoyl]glutamic acid" (20b)

Yield, $72 \%$, Orange powder, m.p. $246-248{ }^{\circ} \mathrm{C}$. IR, $3442-3421 \mathrm{~cm}^{-1}(2 \mathrm{OH}), 3325-3212 \mathrm{~cm}^{-1}$ (6NH), $2975 \mathrm{~cm}^{-1}$ (Ar-H), $2857 \mathrm{~cm}^{-1}$ (Aliphatic-H), 1680-1621 $\mathrm{cm}^{-1}(5 \mathrm{C}=\mathrm{O}$ and $\mathrm{C}=\mathrm{N})$. ${ }^{1} \mathrm{H}-\mathrm{NMR}$ (DMSO $\left.d_{6}, 850 \mathrm{MHz}\right): \delta=1.91-2.05\left(\mathrm{~m}, 2 \mathrm{H}, \mathrm{CH}_{2} \mathrm{CH}_{2} \mathrm{COOH}\right), 2.54(\mathrm{t}, 2 \mathrm{H}$, $\left.\mathrm{CH}_{2} \mathrm{CH}_{2} \mathrm{COOH}\right), 2.43\left(\mathrm{~s}, 6 \mathrm{H}, 2 \mathrm{CH}_{3}\right), 4.37(\mathrm{t}, 1 \mathrm{H}, \mathrm{NHC} \underline{\mathrm{HCOH}}), 4.52(\mathrm{~s}, 2 \mathrm{H}$, pteridine$\left.\mathrm{CH}_{2}-\mathrm{N}\right), 6.45\left(\mathrm{~d}, 2 \mathrm{H}, J=8.4 \mathrm{~Hz}\right.$, benzene $\left.\mathrm{C}_{2} \underline{H}, \mathrm{C}_{6} \underline{H}\right), 6.68\left(\mathrm{~d}, 2 \mathrm{H}, \mathrm{N}-\mathrm{Ph}-(\underline{H})_{\text {ortho }}\right), 6.83(\mathrm{~d}, 2 \mathrm{H}$, $J=8.4 \mathrm{~Hz}$, benzene $\left.\mathrm{C}_{3} \underline{H}, \mathrm{C}_{5} \underline{H}\right), 6.89(\mathrm{~s}, 1 \mathrm{H}, \underline{H \mathrm{~N}}-\mathrm{Ph}), 7.61(\mathrm{~m}, 2 \mathrm{H}, \mathrm{N}-\mathrm{Ph}-(\underline{H})$ (meta) $), 8.13$ (s, $1 \mathrm{H}, \mathrm{N} \underline{H C O}), 8.40\left(\mathrm{t}, 1 \mathrm{H}\right.$, pyrimidine $\left.\mathrm{C}_{3} \underline{H}\right), 8.55(\mathrm{~s}, 1 \mathrm{H}$, pteridine- $\underline{\mathrm{HCONH}}), 8.61(\mathrm{~d}, 2 \mathrm{H}$, pyrimidine $\left.\mathrm{C}_{2} \underline{H}, \mathrm{C}_{4} \underline{H}\right), 8.79\left(\mathrm{~s}, 1 \mathrm{H}\right.$, pteridine- $\left.\mathrm{C}_{7} \underline{H}\right), 8.91(\mathrm{~s}, 1 \mathrm{H}$, pteridine-NHCON$\underline{H}), 10.31$ (s, $1 \mathrm{H}$, pteridine $\mathrm{N} \underline{H}), 11.31\left(\mathrm{~s}, 1 \mathrm{H}, \mathrm{CH}_{2} \mathrm{CH}_{2} \mathrm{COO} \underline{H}\right), 12.34\left(\mathrm{~s}, 1 \mathrm{H}, \mathrm{CH}_{2} \mathrm{COO} \underline{H}\right), 12.85(\mathrm{~s}, 1 \mathrm{H}$, $\mathrm{SO}_{2} \mathrm{~N} \underline{H}$ ). Anal. Calcd. for $\mathrm{C}_{32} \mathrm{H}_{31} \mathrm{~N}_{11} \mathrm{O}_{9} \mathrm{~S}$ (745.72): $\mathrm{C}, 51.54 ; \mathrm{H}, 4.19 ; \mathrm{N}, 20.66 ; \mathrm{S}, 4.30$; found: C, 51.21; H, 4.02; N, 20.49; S, 4.17.

4.3.3. “N-[4-(\{[2-(\{[2-(aminocarbonyl)hydrazino]methylene\}-amino)-4-oxo-3,4dihydropteridin-6-yl]methyl \}amino)benzoyl]glutamic acid" (21)

Compound 4 (0.001 mol, $0.5 \mathrm{~g})$ and semicarbazide $\mathrm{HCl}(0.001 \mathrm{~mol}, 0.11 \mathrm{~g})$ and drops of TMA in DMF $(14 \mathrm{~mL})$ was stirred under reflux for $4 \mathrm{~h}$ (TLC, $R_{F}=0.65$, eluent: $\mathrm{CH}_{2} \mathrm{Cl}_{2}$ ). Brown powder formed after crystallization from EtOH. Yield 78\%, m.p. 250-252 ${ }^{\circ} \mathrm{C}$. IR, 3412-3389 $\mathrm{cm}^{-1}(2 \mathrm{OH}), 3329-33251 \mathrm{~cm}^{-1}\left(\mathrm{NH}_{2}\right.$ and $\left.5 \mathrm{NH}\right), 2949 \mathrm{~cm}^{-1}$ (Ar-H), $2850 \mathrm{~cm}^{-1}$ (Aliphatic-H), $1666-1623 \mathrm{~cm}^{-1}(5 \mathrm{C}=\mathrm{O}$ and $\mathrm{C}=\mathrm{N}) .{ }^{1} \mathrm{H}-\mathrm{NMR}$ (DMSO $\left.d_{6}, 850 \mathrm{MHz}\right): \delta=1.91-2.05\left(\mathrm{~m}, 2 \mathrm{H}, \mathrm{CH}_{2} \mathrm{CH}_{2} \mathrm{COOH}\right), 2.54\left(\mathrm{t}, 2 \mathrm{H}, \mathrm{CH}_{2} \mathrm{CH}_{2} \mathrm{COOH}\right), 4.31$ $(\mathrm{t}, 1 \mathrm{H}, \mathrm{NHC} \underline{\mathrm{HCOOH}}), 4.44\left(\mathrm{~s}, 2 \mathrm{H}\right.$, pteridine- $\left.\mathrm{CH}_{2}-\mathrm{N}\right), 6.64\left(\mathrm{~d}, 2 \mathrm{H}, \mathrm{N}-\mathrm{Ph}-(\underline{\mathrm{H}}){ }_{\text {ortho }}\right), 6.89$ (s,

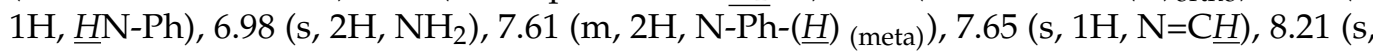
$1 \mathrm{H}, \mathrm{N} \underline{H} \mathrm{CO}), 8.84\left(\mathrm{~s}, 1 \mathrm{H}\right.$, pteridine- $\left.\mathrm{C}_{7} \underline{H}\right), 10.31(\mathrm{~s}, 1 \mathrm{H}$, pteridine $\mathrm{N} \underline{H}), 10.57(\mathrm{~s}, 1 \mathrm{H}, \mathrm{N} \underline{H}-\mathrm{NH})$, 10.99 (s, $1 \mathrm{H}, \mathrm{NH}-\mathrm{N} \underline{H}), 11.41$ (s, $\left.1 \mathrm{H}, \mathrm{CH}_{2} \mathrm{CH}_{2} \mathrm{COO} \underline{H}\right), 12.28\left(\mathrm{~s}, 1 \mathrm{H}, \mathrm{CH}_{2} \mathrm{COO} \underline{H}\right)$. Anal. Calcd. for $\mathrm{C}_{21} \mathrm{H}_{22} \mathrm{~N}_{10} \mathrm{O}_{7}$ (526.46): $\mathrm{C}, 47.91 ; \mathrm{H}, 4.21 ; \mathrm{N}, 26.61$; found: $\mathrm{C}, 47.63 ; \mathrm{H}, 3.92 ; \mathrm{N}, 26.47$.

4.3.4. “ $N-\{4-[(\{2-[(\{2-[a m i n o(i m i n o) m e t h y l] h y d r a z i n o\}-m e t h y l e n e) a m i n o]-4-o x o-3,4-$ dihydropteridin-6-yl\}methyl)amino]-benzoyl\}glutamic acid" (22)

Compound 14 (0.001 mol, $0.5 \mathrm{~g})$ and aminoguanidinium hydrocarbonate $(0.001 \mathrm{~mol}$, $0.14 \mathrm{~g})$ in glacial $\mathrm{AcOH}(15 \mathrm{~mL})$ was stirred under reflux (TLC, $R_{F}=0.6$, eluent: $\mathrm{CH}_{2} \mathrm{Cl}_{2}$ ), after $2 \mathrm{~h}$ a green precipitate formed, which crystallized from EtOH. Yield 96\%, m.p. 278$280{ }^{\circ} \mathrm{C}$. IR, 3442-3415 cm $\mathrm{cm}^{-1}(2 \mathrm{OH}), 3289-3151 \mathrm{~cm}^{-1}\left(\mathrm{NH}_{2}\right.$ and $\left.6 \mathrm{NH}\right), 2951 \mathrm{~cm}^{-1}$ (Ar-H), $2847 \mathrm{~cm}^{-1}$ (Aliphatic-H), 1672-1617 $\mathrm{cm}^{-1}(4 \mathrm{C}=\mathrm{O}$ and $\mathrm{C}=\mathrm{N}) .{ }^{1} \mathrm{H}-\mathrm{NMR}$ (DMSO $d_{6}, 850$ $\mathrm{MHz}): \delta=1.93-2.05\left(\mathrm{~m}, 2 \mathrm{H}, \mathrm{CH}_{2} \mathrm{CH}_{2} \mathrm{COOH}\right), 2.52\left(\mathrm{t}, 2 \mathrm{H}, \mathrm{CH}_{2} \mathrm{CH}_{2} \mathrm{COOH}\right), 4.29(\mathrm{t}, 1 \mathrm{H}$, $\mathrm{NHC} \underline{\mathrm{HCOOH}}), 4.51\left(\mathrm{~s}, 2 \mathrm{H}\right.$, pteridine- $\left.\mathrm{CH}_{2}-\mathrm{N}\right), 6.21\left(\mathrm{~s}, 2 \mathrm{H}, \mathrm{NH}_{2}\right), 6.61\left(\mathrm{~d}, 2 \mathrm{H}, \mathrm{N}-\mathrm{Ph}-(\underline{\mathrm{H}})_{\text {ortho }}\right)$, $6.90(\mathrm{~s}, 1 \mathrm{H}, \underline{H \mathrm{~N}}-\mathrm{Ph}), 7.60\left(\mathrm{~m}, 2 \mathrm{H}, \mathrm{N}-\mathrm{Ph}-(\underline{H}){ }_{(\text {meta })}\right), 7.55(\mathrm{~s}, 1 \mathrm{H}, \mathrm{N}=\mathrm{C} \underline{H}), 7.82(\mathrm{~s}, 1 \mathrm{H}, \mathrm{C}=\mathrm{NH})$, $8.11(\mathrm{~s}, 1 \mathrm{H}, \mathrm{N} \underline{H} \mathrm{CO}), 8.82\left(\mathrm{~s}, 1 \mathrm{H}\right.$, pteridine- $\left.\mathrm{C}_{7} \underline{H}\right), 10.13(\mathrm{~s}, 1 \mathrm{H}, \mathrm{N} \underline{H}-\mathrm{NH}), 10.33(\mathrm{~s}, 1 \mathrm{H}$, pteridine $\mathrm{N} \underline{H}), 10.90$ (s, 1H, NH-N $\underline{H}), 11.42\left(\mathrm{~s}, 1 \mathrm{H}, \mathrm{CH}_{2} \mathrm{CH}_{2} \mathrm{COO} \underline{H}\right), 12.31$ (s,1H, $\left.\mathrm{CH}_{2} \mathrm{COO} \underline{H}\right)$. Anal. Calcd. for $\mathrm{C}_{21} \mathrm{H}_{23} \mathrm{~N}_{11} \mathrm{O}_{6}$ (525.48): $\mathrm{C}, 48.00 ; \mathrm{H}, 4.41 ; \mathrm{N}, 29.32$; found: $\mathrm{C}, 47.61 ; \mathrm{H}, 4.28 ; \mathrm{N}, 29.10$. 
4.3.5. “N-[4-(\{[2-(\{[(carboxymethyl)amino]methylene $\} a m i n o)-4-o x o-3,4-d i h y d r o-p t e r i d i n-$ 6-yl]methyl\}amino)benzoyl]glutamic acid" (23)

A mixture of compound 14 (0.001 mol, $0.5 \mathrm{~g})$ and glycine $(0.001 \mathrm{~mol}, 0.08 \mathrm{~g})$ in DMF/ $\mathrm{H}_{2} \mathrm{O}$ mixture $\left(9: 1,10 \mathrm{~mL}\right.$ ) refluxed for $3 \mathrm{~h}$ (TLC, $R_{F}=0.75$, eluent: $\mathrm{CH}_{2} \mathrm{Cl}_{2}$ ). The brown solid formed crystallized from EtOH to give crystals. Yield, 91\%, m.p. $218-220{ }^{\circ} \mathrm{C}$. IR, $3443-3405 \mathrm{~cm}^{-1}(3 \mathrm{OH}), 3317-3122 \mathrm{~cm}^{-1}$ (4NH), $2910 \mathrm{~cm}^{-1}$ (Ar-H), $2850 \mathrm{~cm}^{-1}$ (Aliphatic$\mathrm{H}), 1688-1619 \mathrm{~cm}^{-1}(5 \mathrm{C}=\mathrm{O}$ and $\mathrm{C}=\mathrm{N}) .{ }^{1} \mathrm{H}-\mathrm{NMR}\left(\mathrm{DMSO} d_{6}, 850 \mathrm{MHz}\right): \delta=1.92-2.03(\mathrm{~m}$, $\left.2 \mathrm{H}, \mathrm{CH}_{2} \mathrm{CH}_{2} \mathrm{COOH}\right), 2.54\left(\mathrm{t}, 2 \mathrm{H}, \mathrm{CH}_{2} \mathrm{CH}_{2} \mathrm{COOH}\right), 4.18\left(\mathrm{~s}, 2 \mathrm{H}, \mathrm{NHCH} \mathrm{COOH}_{2}, 4.33(\mathrm{t}\right.$, $1 \mathrm{H}, \mathrm{NHC} \underline{\mathrm{HCOOH}}), 4.55$ (s, 2H, pteridine- $\left.\overline{\mathrm{C}} \mathrm{H}_{2}-\mathrm{N}\right), 6.63\left(\mathrm{~d}, 2 \mathrm{H}, \mathrm{N}-\mathrm{Ph}-(\underline{\mathrm{H}})_{\text {ortho }}\right), 6.90(\mathrm{~s}, 1 \mathrm{H}$,

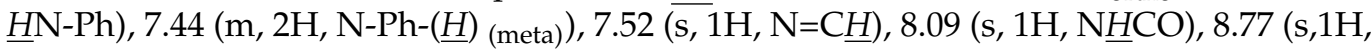
pteridine $\left.-\mathrm{C}_{7} \underline{\mathrm{H}}\right), 8.96\left(\mathrm{~s}, 1 \mathrm{H}, \mathrm{N} \underline{\mathrm{H} C \mathrm{H}_{2} \mathrm{COOH}}\right), 10.36(\mathrm{~s}, 1 \mathrm{H}$, pteridine $\mathrm{NH}), 11.34(\mathrm{~s}, 1 \mathrm{H}$, $\left.\mathrm{CH}_{2} \mathrm{CH}_{2} \mathrm{COO} \underline{H}\right), 11.58\left(\mathrm{~s}, 1 \mathrm{H}, \mathrm{CH}_{2} \mathrm{COO} \underline{H}\right), 12.28$ (s, $\left.1 \mathrm{H}, \mathrm{NHCH}_{2} \mathrm{COO} \underline{H}\right)$. Anal. Calcd. for $\mathrm{C}_{22} \mathrm{H}_{22} \mathrm{~N}_{8} \mathrm{O}_{8}$ (526.46): C, 50.19; H, 4.21; N, 21.28; found: C, 49.84; H, 4.06; N, 21.01.

\subsection{Reaction of Compound $\mathbf{1 4}$ and Sulfa Drugs (24a,b): General Procedure}

A mixture of 14 (0.001 mol, $0.5 \mathrm{~g})$ and appropriate sulfa drug (0.001 mol) in DMF $(15 \mathrm{~mL})$ was stirred under reflux for $3 \mathrm{~h}$ (TLC, $\mathrm{R}_{F}=0.4$, eluent: $\mathrm{CH}_{2} \mathrm{Cl}_{2}$ ). The precipitate formed crystallized from EtOH to yield brownish powder.

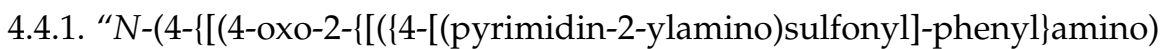
methylene]-amino\}-3,4-dihydropteridin-6-yl)methyl]amino\}-benzoyl)-glutamic acid" (24a)

Brownish powder, yield, $76 \%$, m.p. $236-238{ }^{\circ} \mathrm{C}$. IR, $3456-3412 \mathrm{~cm}^{-1}(2 \mathrm{OH}), 3321-$ $3228 \mathrm{~cm}^{-1}$ (5NH), $2974 \mathrm{~cm}^{-1}$ (Ar-H), $2803 \mathrm{~cm}^{-1}$ (Aliphatic-H), 1687-1616 $\mathrm{cm}^{-1}(4 \mathrm{C}=\mathrm{O}$ and $\mathrm{C}=\mathrm{N}$ ). ${ }^{1} \mathrm{H}-\mathrm{NMR}\left(\mathrm{DMSO} d_{6}, 850 \mathrm{MHz}\right): \delta=1.93-2.05\left(\mathrm{~m}, 2 \mathrm{H}, \mathrm{CH}_{2} \mathrm{CH}_{2} \mathrm{COOH}\right), 2.53$ $\left(\mathrm{t}, 2 \mathrm{H}, \mathrm{CH}_{2} \mathrm{CH}_{2} \mathrm{COOH}\right), 4.32(\mathrm{t}, 1 \mathrm{H}, \mathrm{NHCHCOOH}), 4.52\left(\mathrm{~s}, 2 \mathrm{H}\right.$, pteridine- $\left.\mathrm{CH}_{2}-\mathrm{N}\right), 6.41$ $\left(\mathrm{d}, 2 \mathrm{H}, J=8 . \overline{4 \mathrm{~Hz}}\right.$, benzene $\left.\mathrm{C}_{2} \underline{H}, \mathrm{C}_{6} \underline{H}\right), 6.61\left(\mathrm{~d}, 2 \mathrm{H}, \mathrm{N}-\mathrm{Ph}-(\underline{H})_{\text {ortho }}\right), 6.84(\mathrm{~d}, \overline{2 \mathrm{H}}, J=8.4$ $\mathrm{Hz}$, benzene $\left.\mathrm{C}_{3} \underline{H}, \mathrm{C}_{5} \underline{H}\right), 6.91(\mathrm{~s}, 1 \mathrm{H}, \underline{H} \mathrm{~N}-\mathrm{Ph}), 7.02(\mathrm{~s}, 1 \mathrm{H}, \mathrm{N}=\mathrm{CH}-\mathrm{N} \underline{H}), 7.52(\mathrm{~s}, 1 \mathrm{H}, \mathrm{N}=\mathrm{C} \underline{H})$, $7.64\left(\mathrm{~m}, 2 \mathrm{H}, \mathrm{N}-\mathrm{Ph}-(\underline{H}){ }_{(\text {meta })}\right), 8.11(\mathrm{~s}, 1 \mathrm{H}, \mathrm{N} \underline{H C O}), 8.45\left(\mathrm{t}, 1 \mathrm{H}\right.$, pyrimidine $\left.\mathrm{C}_{3} \underline{H}\right), 8.62(\mathrm{~d}$, $2 \mathrm{H}$, pyrimidine $\left.\mathrm{C}_{2} \underline{H}, \mathrm{C}_{4} \underline{H}\right), 8.79\left(\mathrm{~s}, 1 \mathrm{H}\right.$, pteridine $\left.-\mathrm{C}_{7} \underline{H}\right), 10.29(\mathrm{~s}, 1 \mathrm{H}$, pteridine $\mathrm{NH}), 11.44$ (s, $\left.1 \mathrm{H}, \mathrm{CH}_{2} \mathrm{CH}_{2} \mathrm{COO} \underline{H}\right), 12.28\left(\mathrm{~s}, 1 \mathrm{H}, \mathrm{CH}_{2} \mathrm{COO} \underline{H}\right), 12.87$ (s, $\left.1 \mathrm{H}, \mathrm{SO}_{2} \mathrm{~N} \underline{H}\right)$. Anal. Calcd. for $\mathrm{C}_{30} \mathrm{H}_{27} \mathrm{~N}_{11} \mathrm{O}_{8} \mathrm{~S}$ (701.67): C, 51.35; H, 3.88; N, 21.96; S, 4.57; found: C, 51.02; H, 3.42; N, 21.71, S, 4.33 .

4.4.2. “N-[4-(\{[2-(\{[(4-\{[(4,6-dimethylpyrimidin-2-yl)amino]-sulfonyl $\}$ phenyl)amino]methylene\}amino)-4-oxo-3,4-dihydropteridin-6-yl]methyl\}-amino)benzoyl]glutamic acid" $^{\prime \prime}(24 b)$

Brown powder, yield, 74\%, m.p. $258-260{ }^{\circ} \mathrm{C}$. IR, 3453-3405 $\mathrm{cm}^{-1}$ (2OH), 3314-3212 $\mathrm{cm}^{-1}(5 \mathrm{NH}), 2952 \mathrm{~cm}^{-1}$ (Ar-H), $2851 \mathrm{~cm}^{-1}$ (Aliphatic-H), 1692-1621 cm $\mathrm{cm}^{-1}$ (4C=O and $\mathrm{C}=\mathrm{N}) .{ }^{1} \mathrm{H}-\mathrm{NMR}\left(\mathrm{DMSO} d_{6}, 850 \mathrm{MHz}\right): \delta=1.90-2.04\left(\mathrm{~m}, 2 \mathrm{H}, \mathrm{CH}_{2} \mathrm{CH}_{2} \mathrm{COOH}\right), 2.51(\mathrm{t}, 2 \mathrm{H}$, $\left.\mathrm{CH}_{2} \mathrm{CH}_{2} \mathrm{COOH}\right), 2.68\left(\mathrm{~s}, 6 \mathrm{H}, 2 \mathrm{CH}_{3}\right), 4.30(\mathrm{t}, 1 \mathrm{H}, \mathrm{NHC} \underline{\mathrm{HCOOH}}), 4.52$ (s, $2 \mathrm{H}$, pteridine$\left.\mathrm{CH}_{2}-\mathrm{N}\right), 6.41\left(\mathrm{~d}, 2 \mathrm{H}, J=8.4 \mathrm{~Hz}\right.$, benzene $\left.\mathrm{C}_{2} \underline{\mathrm{H}}, \mathrm{C}_{6} \underline{H}\right), 6.64\left(\mathrm{~d}, 2 \mathrm{H}, \mathrm{N}-\mathrm{Ph}-(\underline{H})_{\text {ortho }}\right), 6.80(\mathrm{~d}, 2 \mathrm{H}$, $J=8.4 \mathrm{~Hz}$, benzene $\left.\mathrm{C}_{3} \underline{H}, \mathrm{C}_{5} \underline{H}\right), 6.91(\mathrm{~s}, 1 \mathrm{H}, \underline{H} \underline{\mathrm{N}}-\mathrm{Ph}), 7.03(\mathrm{~s}, 1 \mathrm{H}, \mathrm{N}=\mathrm{CH}-\mathrm{N} \underline{H}), 7.53(\mathrm{~s}, 1 \mathrm{H}$, $\mathrm{N}=\mathrm{C} \underline{H}), 7.61\left(\mathrm{~m}, 2 \mathrm{H}, \mathrm{N}-\mathrm{Ph}-(\underline{H}){ }_{(\text {meta })}\right), 8.11(\mathrm{~s}, 1 \mathrm{H}, \mathrm{N} \underline{H C O}), 8.44\left(\mathrm{t}, 1 \mathrm{H}\right.$, pyrimidine $\left.\mathrm{C}_{3} \underline{H}\right)$, $8.61\left(\mathrm{~d}, 2 \mathrm{H}\right.$, pyrimidine $\left.\mathrm{C}_{2} \underline{H}, \mathrm{C}_{4} \underline{H}\right), 8.80\left(\mathrm{~s}, 1 \mathrm{H}\right.$, pteridine- $\left.\mathrm{C}_{7} \underline{H}\right), 10.33(\mathrm{~s}, 1 \mathrm{H}$, pteridine $\mathrm{NH})$, 11.45 (s, $\left.1 \mathrm{H}, \mathrm{CH}_{2} \mathrm{CH}_{2} \mathrm{COO} \underline{H}\right), 12.31\left(\mathrm{~s}, 1 \mathrm{H}, \mathrm{CH}_{2} \mathrm{COO} \underline{H}\right), 12.84$ (s, $\left.1 \mathrm{H}, \mathrm{SO}_{2} \mathrm{~N} \underline{H}\right)$. Anal. Calcd. for $\mathrm{C}_{32} \mathrm{H}_{31} \mathrm{~N}_{11} \mathrm{O}_{8} \mathrm{~S}$ (729.72): C, 52.67; H, 4.28; N, 21.11; S, 4.39; found: C, 52.40; H, 4.10; N, $21.03 ; \mathrm{S}, 4.13$.

4.4.3. “N-(4-\{[(4-amino-3-mercapto-2,12-dioxo-5-phenyl-1,12-dihydro-2Hpyrido[3',2':5,6]-pyrimido[2,1-b]pteridin-10-yl)methyl]-amino\}benzoyl)-glutamic acid"

Compound 10 (0.001 mol, $0.59 \mathrm{~g})$, thioglycolic acid $(0.001 \mathrm{~mol}, 0.09 \mathrm{~g})$, and drops of TMA in EtOH (12 mL) were refluxed for $15 \mathrm{~h}$ (TLC, $\mathrm{R}_{F}=0.55$, eluent: $\mathrm{CH}_{2} \mathrm{Cl}_{2}$ ). The precipitate formed crystallized from DMF/EtOH 1:1 to yield dark orange crystals. Yield, 76\%, m.p. 
251-253 ${ }^{\circ} \mathrm{C}$. IR, 3401-3389 $\mathrm{cm}^{-1}(2 \mathrm{OH}), 3287-3165 \mathrm{~cm}^{-1}\left(\mathrm{NH}_{2}, 3 \mathrm{NH}\right), 2947 \mathrm{~cm}^{-1}$ (Ar-H), $2852 \mathrm{~cm}^{-1}$ (Aliphatic-H), $2657 \mathrm{~cm}^{-1}$ (SH), $1683-1622 \mathrm{~cm}^{-1}(5 \mathrm{C}=\mathrm{O}$ and $\mathrm{C}=\mathrm{N}) .{ }^{1} \mathrm{H}-\mathrm{NMR}$ (DMSO $\left.d_{6}, 850 \mathrm{MHz}\right): \delta=1.90-2.03\left(\mathrm{~m}, 2 \mathrm{H}, \mathrm{CH}_{2} \mathrm{CH}_{2} \mathrm{COOH}\right), 2.55\left(\mathrm{t}, 2 \mathrm{H}, \mathrm{CH}_{2} \mathrm{CH}_{2} \mathrm{COOH}\right)$, $4.06(\mathrm{t}, 1 \mathrm{H}, \mathrm{NHC} \underline{\mathrm{HCOOH}}), 4.53\left(\mathrm{~s}, 2 \mathrm{H}\right.$, pteridine- $\left.\mathrm{CH}_{2}-\mathrm{N}\right), 6.92\left(\mathrm{~d}, 2 \mathrm{H}, \mathrm{N}-\mathrm{Ph}-(\underline{\mathrm{H}})_{\text {ortho }}\right), 6.96$ (s, $1 \mathrm{H}, \underline{H \mathrm{~N}}-\mathrm{Ph}), 7.68-7.90\left(\mathrm{~m}, 7 \mathrm{H}, \mathrm{N}-\mathrm{Ph}-(\underline{H})_{(\text {meta) }}\right.$ and pyrimidine-4-Ph), $8.12(\mathrm{~s}, 1 \mathrm{H}, \mathrm{N} \underline{H C O})$, $8.67\left(\mathrm{~s}, 1 \mathrm{H}\right.$, pteridine- $\left.\mathrm{C}_{7} \underline{H}\right), 11.38(\mathrm{~s}, 1 \mathrm{H}$, pyridine- $\mathrm{N} \underline{H}), 11.44\left(\mathrm{~s}, 1 \mathrm{H}, \mathrm{CH}_{2} \mathrm{CH}_{2} \mathrm{COO} \underline{H}\right), 12.31$ (s,1H, $\left.\mathrm{CH}_{2} \mathrm{COO} \underline{H}\right), 14.01$ (s, $1 \mathrm{H}$, pyridine- $\mathrm{S} \underline{\mathrm{H}}$ ). Anal. Calcd. for $\mathrm{C}_{31} \mathrm{H}_{25} \mathrm{~N}_{9} \mathrm{O}_{7} \mathrm{~S}$ (667.65): $\mathrm{C}$, 55.77; H, 3.77; N, 18.88; S, 4.80; found: C, 55.54; H, 3.62; N, 18.64; S, 4.67.

4.4.4. “ $N-\{4-[(\{8-c y a n o-9-[(m e r c a p t o a c e t y l) a m i n o]-11-o x o-7-p h e n y l-11 H-p y r i m i d o[2,1-$ b]pteridin-2-yl\}methyl)amino]benzoyl\}glutamic acid" (26)

Compound 10 (0.001 mol, $0.59 \mathrm{~g})$ and thioglycolic acid (0.001 mol, $0.09 \mathrm{~g})$ in $\mathrm{EtOH}$ $\left(13 \mathrm{~mL}\right.$ ) were refluxed for $5 \mathrm{~h}$ (TLC, $R_{F}=0.5$, eluent: $\mathrm{CH}_{2} \mathrm{Cl}_{2}$ ). The precipitate formed on hot filtered and crystallized from DMF/EtOH 1:1 to produce yellowish brown crystals. Yield, $89 \%$, m.p. $238-240{ }^{\circ} \mathrm{C}$. IR, $3427-3412 \mathrm{~cm}^{-1}(2 \mathrm{OH}), 3248-3153 \mathrm{~cm}^{-1}(3 \mathrm{NH}), 2961 \mathrm{~cm}^{-1}$ (Ar-H), $2866 \mathrm{~cm}^{-1}$ (Aliphatic-H), $2634 \mathrm{~cm}^{-1}$ (SH), $2206 \mathrm{~cm}^{-1}$ (CN), 1678-1618 cm-1 (5C=O and $\mathrm{C}=\mathrm{N}$ ). ${ }^{1} \mathrm{H}-\mathrm{NMR}$ (DMSO $\left.d_{6}, 850 \mathrm{MHz}\right): \delta=1.91-2.07\left(\mathrm{~m}, 2 \mathrm{H}, \mathrm{CH}_{2} \mathrm{CH}_{2} \mathrm{COOH}\right), 2.53$ $\left(\mathrm{t}, 2 \mathrm{H}, \mathrm{CH}_{2} \mathrm{CH}_{2} \mathrm{COOH}\right), 3.64\left(\mathrm{~s}, 2 \mathrm{H}, \mathrm{NHCOCH} \mathrm{H}_{2} \mathrm{SH}\right), 4.06(\mathrm{t}, 1 \mathrm{H}, \mathrm{NHC}+\mathrm{HCOOH}), 4.52(\mathrm{~s}$, $2 \mathrm{H}$, pteridine- $\left.\mathrm{CH}_{2}-\mathrm{N}\right), 6.90\left(\mathrm{~d}, 2 \mathrm{H}, \mathrm{N}-\mathrm{Ph}-(\underline{H})_{\text {ortho }}\right), 6.99(\mathrm{~s}, 1 \mathrm{H}, \underline{H \mathrm{~N}}-\mathrm{Ph}), 7.61-7.91(\mathrm{~m}, 7 \mathrm{H}$, $\mathrm{N}-\mathrm{Ph}-(\underline{\mathrm{H}})_{(\text {meta) }}$ and pyrimidine-4-Ph), $8.19(\mathrm{~s}, 1 \mathrm{H}, \mathrm{N} \underline{H C O}), 8.66\left(\mathrm{~s}, 1 \mathrm{H}\right.$, pteridine- $\left.\mathrm{C}_{7} \underline{\mathrm{H}}\right), 10.37$ (s, $\left.1 \mathrm{H}, \mathrm{NHCOCH}_{2} \mathrm{SH}\right), 11.44$ (s, $\left.1 \mathrm{H}, \mathrm{CH}_{2} \mathrm{CH}_{2} \mathrm{COO} \underline{H}\right), 12.28\left(\mathrm{~s}, 1 \mathrm{H}, \mathrm{CH}_{2} \mathrm{COO} \underline{H}\right), 13.97$ (s, $1 \mathrm{H}$, $\mathrm{NHCOCH}_{2} \mathrm{~S} \underline{\mathrm{H}}$ ). Anal. Calcd. for $\mathrm{C}_{31} \mathrm{H}_{25} \mathrm{~N}_{9} \mathrm{O}_{7} \mathrm{~S}$ (667.65): $\mathrm{C}, 55.77 ; \mathrm{H}, 3.77 ; \mathrm{N}, 18.88 ; \mathrm{S}, 4.80$; found: $\mathrm{C}, 55.54 ; \mathrm{H}, 3.62 ; \mathrm{N}, 18.64 ; \mathrm{S}, 4.67$.

4.4.5. “ $N$ - $\{4-[(\{8-c y a n o-9-[($ mercaptocarbonothioyl $)$ amino $]-11-o x o-7-p h e n y l-11 \mathrm{H}-$ pyrimido-[2,1-b]pteridin-2-yl\}methyl)amino]benzoyl\}-glutamic acid" (27)

Compound $10(0.001 \mathrm{~mol}, 0.59 \mathrm{~g}), \mathrm{CS}_{2}$ (excess, $\left.1 \mathrm{~mL}\right)$ and $\mathrm{KOH}(0.003 \mathrm{~mol}, 0.17 \mathrm{~g})$ in EtOH $(20 \mathrm{~mL})$ was refluxed for $5 \mathrm{~h}\left(\mathrm{TLC}, R_{F}=0.35\right.$, eluent: $\left.\mathrm{CH}_{2} \mathrm{Cl}_{2}\right)$. The solution poured onto crushed ice after concentration then acidified with dilute $\mathrm{HCl}$. The precipitate formed crystallized from EtOH to produce reddish brown powder. Yield, $57 \%$, m.p. $240-242{ }^{\circ} \mathrm{C}$. IR, $3418-3406 \mathrm{~cm}^{-1}(2 \mathrm{OH}), 3221-3170 \mathrm{~cm}^{-1}(3 \mathrm{NH}), 2950 \mathrm{~cm}^{-1}$ (Ar-H), $2838 \mathrm{~cm}^{-1}$ (Aliphatic$\mathrm{H}), 2630 \mathrm{~cm}^{-1}(\mathrm{SH}), 2202 \mathrm{~cm}^{-1}(\mathrm{CN}), 1689-1621 \mathrm{~cm}^{-1}(4 \mathrm{C}=\mathrm{O}$ and $\mathrm{C}=\mathrm{N}), 1328 \mathrm{~cm}^{-1}$ $(\mathrm{C}=\mathrm{S}) .{ }^{1} \mathrm{H}-\mathrm{NMR}\left(\mathrm{DMSO} d_{6}, 850 \mathrm{MHz}\right): \delta=1.92-2.04\left(\mathrm{~m}, 2 \mathrm{H}, \mathrm{CH}_{2} \mathrm{CH}_{2} \mathrm{COOH}\right), 2.54(\mathrm{t}$, $\left.2 \mathrm{H}, \mathrm{CH}_{2} \mathrm{CH}_{2} \mathrm{COOH}\right), 4.05(\mathrm{t}, 1 \mathrm{H}, \mathrm{NHC} \underline{\mathrm{HCOOH}}), 4.41\left(\mathrm{~s}, 2 \mathrm{H}\right.$, pteridine- $\left.\mathrm{CH}_{2}-\mathrm{N}\right), 6.90(\mathrm{~d}$, $\left.2 \mathrm{H}, \mathrm{N}-\mathrm{Ph}-(\underline{\underline{H}})_{\text {ortho }}\right), 6.95(\mathrm{~s}, 1 \mathrm{H}, \underline{H \mathrm{~N}}-\mathrm{Ph}), 7.61-7.95(\mathrm{~m}, 7 \mathrm{H}, \mathrm{N}-\mathrm{Ph}-(\underline{H})$ (meta) and pyrimidine4- $P h), 8.18(\mathrm{~s}, 1 \mathrm{H}, \mathrm{N} \underline{H C O}), 8.66\left(\mathrm{~s}, 1 \mathrm{H}\right.$, pteridine $\left.-\mathrm{C}_{7} \underline{H}\right), 10.56(\mathrm{~s}, 1 \mathrm{H}, \mathrm{N} \underline{H} \mathrm{C}=\mathrm{S}) ; 11.41(\mathrm{~s}, 1 \mathrm{H}$, $\left.\mathrm{CH}_{2} \mathrm{CH}_{2} \mathrm{COO} \underline{H}\right), 12.29\left(\mathrm{~s}, 1 \mathrm{H}, \mathrm{CH}_{2} \mathrm{COO} \underline{H}\right), 13.98(\mathrm{~s}, 1 \mathrm{H}, \mathrm{S} \underline{\mathrm{H}})$. Calcd. for $\mathrm{C}_{30} \mathrm{H}_{23} \mathrm{~N}_{9} \mathrm{O}_{6} \mathrm{~S}_{2}$ (669.69): C, 53.80; H, 3.46; N, 18.82; S, 9.58; found: C, 53.66; H, 3.39; N, 18.71; S, 9.42.

4.4.6. “N-(4-\{[(12-oxo-5-phenyl-2,4-dithioxo-1,3,4,12-tetrahydro-2H-pyrimido-[5', $\left.4^{\prime}: 5,6\right]-$ pyrimido[2,1-b]pteridin-10-yl)methyl]- amino\}benzoyl)glutamic acid" (28)

Compound 10 (0.001 mol, $0.59 \mathrm{~g}), \mathrm{CS}_{2}$ (excess, $1 \mathrm{~mL}$ ) and $\mathrm{KOH}(0.003 \mathrm{~mol}, 0.17 \mathrm{~g}, 3$ mmol) dry pyridine $(10 \mathrm{~mL})$ was refluxed for $12 \mathrm{~h}\left(\mathrm{TLC}, R_{F}=0.5\right.$, eluent: $\left.\mathrm{CH}_{2} \mathrm{Cl}_{2}\right)$. The solution poured onto crushed ice/dilute $\mathrm{HCl}$. The product crystallized from $\mathrm{EtOH}$ to yield orange powder. Yield, $57 \%$, m.p. $262-264{ }^{\circ} \mathrm{C}$. IR, $3419-3410 \mathrm{~cm}^{-1}(2 \mathrm{OH}), 3234-3187 \mathrm{~cm}^{-1}$ (4NH), $2971 \mathrm{~cm}^{-1}$ (Ar-H), $2846 \mathrm{~cm}^{-1}$ (Aliphatic-H), $1675-1623 \mathrm{~cm}^{-1}(4 \mathrm{C}=\mathrm{O}$ and $\mathrm{C}=\mathrm{N})$; 1350-1273 (2C=S). ${ }^{1} \mathrm{H}-\mathrm{NMR}$ (DMSO $\left.d_{6}, 850 \mathrm{MHz}\right): \delta=1.93-2.05\left(\mathrm{~m}, 2 \mathrm{H}, \mathrm{CH}_{2} \mathrm{CH}_{2} \mathrm{COOH}\right), 2.54$ $\left(\mathrm{t}, 2 \mathrm{H}, \mathrm{CH}_{2} \mathrm{CH}_{2} \mathrm{COOH}\right), 4.01(\mathrm{t}, 1 \mathrm{H}, \mathrm{NHC}+\mathrm{HCOH}), 4.44\left(\mathrm{~s}, 2 \mathrm{H}\right.$, pteridine- $\left.\overline{\mathrm{CH}}_{2}-\mathrm{N}\right), 6.91(\mathrm{~d}, 2 \mathrm{H}$, $\left.\mathrm{N}-\mathrm{Ph}-(\underline{H})_{\text {ortho }}\right), 6.97(\mathrm{~s}, 1 \mathrm{H}, \underline{H \mathrm{~N}}-\mathrm{Ph}), 7.62-7.90(\mathrm{~m}, 7 \mathrm{H}, \mathrm{N}-\mathrm{Ph}-(\underline{\mathrm{H}})$ (meta) and pyrimidine-4-Ph), 8.22 (s, $1 \mathrm{H}, \mathrm{N} \underline{H C O}), 8.67\left(\mathrm{~s}, 1 \mathrm{H}\right.$, pteridine- $\left.\mathrm{C}_{7} \underline{H}\right), 10.56\left(\mathrm{~s}, 1 \mathrm{H}, \mathrm{N}_{1} \underline{\mathrm{HC}}=\mathrm{S}\right) ; 11.41\left(\mathrm{~s}, 1 \mathrm{H}, \mathrm{CH}_{2} \mathrm{CH}_{2} \mathrm{COO} \underline{H}\right)$, 12.29 (s, $1 \mathrm{H}, \mathrm{CH}_{2} \mathrm{COO} \underline{H}$ ), 12.56 (s, $1 \mathrm{H}, \mathrm{N}_{2} \underline{\mathrm{HC}}=\mathrm{S}$ ). Calcd. for $\mathrm{C}_{30} \mathrm{H}_{23} \mathrm{~N}_{9} \mathrm{O}_{6} \mathrm{~S}_{2}$ (669.69): C, 53.80; $\mathrm{H}$, 3.46; N, 18.82; S, 9.58; found: C, 53.66; H, 3.39; N, 18.71; S, 9.42 . 
4.4.7. “N-(4-\{[(2,4-diamino-3-cyano-12-oxo-5-phenyl-12H-pyrido[ $\left[3^{\prime}, 2^{\prime}: 5,6\right]$ pyrimido[2,1b]pteridin-10-yl)methyl]amino\}benzoyl)-glutamic acid" (29)

Compound 10 (0.001 mol, $0.59 \mathrm{~g})$ with malononitrile $(0.001 \mathrm{~mol}, 0.07 \mathrm{~g})$, and drops of TMA in EtOH (13 mL) refluxed for $13 \mathrm{~h}$ (TLC, $\mathrm{R}_{F}=0.6$, eluent: $\mathrm{CH}_{2} \mathrm{Cl}_{2}$ ). The red product formed crystallized from EtOH to produce dark red powder. Yield, 69\%, m.p. 236-238 ${ }^{\circ} \mathrm{C}$. IR, 3423-3415 cm $\mathrm{cm}^{-1}(2 \mathrm{OH}), 3245-3162 \mathrm{~cm}^{-1}\left(2 \mathrm{NH}_{2}, 2 \mathrm{NH}\right), 2944 \mathrm{~cm}^{-1}(\mathrm{Ar}-\mathrm{H})$, $2852 \mathrm{~cm}^{-1}$ (Aliphatic-H), $2201 \mathrm{~cm}^{-1}(\mathrm{CN}) ; 1678-1621 \mathrm{~cm}^{-1}(4 \mathrm{C}=\mathrm{O}$ and $\mathrm{C}=\mathrm{N}) .{ }^{1} \mathrm{H}-\mathrm{NMR}$ $\left(\mathrm{DMSO} d_{6}, 850 \mathrm{MHz}\right): \delta=1.93-2.04\left(\mathrm{~m}, 2 \mathrm{H}, \mathrm{CH}_{2} \mathrm{CH}_{2} \mathrm{COOH}\right), 2.55\left(\mathrm{t}, 2 \mathrm{H}, \mathrm{CH}_{2} \mathrm{CH}_{2} \mathrm{COOH}\right)$, $4.11(\mathrm{t}, 1 \mathrm{H}, \mathrm{NHC} \underline{\mathrm{HCOOH}}), 4.42\left(\mathrm{~s}, 2 \mathrm{H}\right.$, pteridine- $\left.\mathrm{CH}_{2}-\mathrm{N}\right), 6.12\left(\mathrm{~s}, 2 \mathrm{H}, \mathrm{C}_{2} \mathrm{~N}_{\underline{H_{2}}}\right), 6.58(\mathrm{~s}$, $\left.2 \mathrm{H}, \mathrm{C}_{4} \mathrm{~N}_{2}\right), 6.92$ (d, 2H, N-Ph- $\left.(\underline{H})_{\text {ortho }}\right), 6.99$ (s, 1H, $\left.\underline{\mathrm{HN}}-\mathrm{Ph}\right), 7.62-7.93$ (m, 7H, N-Ph- $(\underline{H})$ (meta) and pyrimidine-4- $P h), 8.21(\mathrm{~s}, 1 \mathrm{H}, \mathrm{N} \underline{\mathrm{HCO}}), 8.61\left(\mathrm{~s}, 1 \mathrm{H}\right.$, pteridine- $\left.\mathrm{C}_{7} \underline{H}\right), 11.43(\mathrm{~s}, 1 \mathrm{H}$, $\left.\mathrm{CH}_{2} \mathrm{CH}_{2} \mathrm{COO} \underline{H}\right), 12.22$ (s, $1 \mathrm{H}, \mathrm{CH}_{2} \mathrm{COO} \underline{H}$ ). Calcd. for $\mathrm{C}_{32} \mathrm{H}_{25} \mathrm{~N}_{11} \mathrm{O}_{6}$ (659.61): C, 58.27; $\mathrm{H}$, 3.82; N, 23.36; found: C, 58.05; H, 3.71; N, 23.23.

4.4.8. “N-(4-\{[(4-amino-12-oxo-5-phenyl-12H-pyrimido-[ $\left[5^{\prime}, 4^{\prime}: 5,6\right]$ pyrimido-[2,1b]pteridin-10-yl)methyl]amino\}benzoyl)glutamic acid" (30)

Compound $10(0.001 \mathrm{~mol}, 0.59 \mathrm{~g})$ refluxed in excess formamide $(8 \mathrm{~mL})$ for $9 \mathrm{~h}$ (TLC, $R_{F}=0.7$, eluent: $\mathrm{CH}_{2} \mathrm{Cl}_{2}$ ). The solution poured on ice-cold water and then extracted with $\mathrm{CH}_{2} \mathrm{Cl}_{2}$ (25 mL, two times) to give yellow product which crystallized from EtOH. Yield, $42 \%$, m.p. $225-227^{\circ} \mathrm{C}$. IR, 3421-3411 cm ${ }^{-1}(2 \mathrm{OH}), 3221-3137 \mathrm{~cm}^{-1}\left(\mathrm{NH}_{2}, 2 \mathrm{NH}\right), 2957 \mathrm{~cm}^{-1}$ $\left(\right.$ Ar-H), $2850 \mathrm{~cm}^{-1}$ (Aliphatic-H), $1671-1623 \mathrm{~cm}^{-1}(4 \mathrm{C}=\mathrm{O}$ and $\mathrm{C}=\mathrm{N}) .{ }^{1} \mathrm{H}-\mathrm{NMR}\left(\mathrm{DMSO} d_{6}\right.$, $850 \mathrm{MHz}): \delta=1.88-2.05\left(\mathrm{~m}, 2 \mathrm{H}, \mathrm{CH}_{2} \mathrm{CH}_{2} \mathrm{COOH}\right), 2.50\left(\mathrm{t}, 2 \mathrm{H}, \mathrm{CH}_{2} \mathrm{CH}_{2} \mathrm{COOH}\right), 4.07(\mathrm{t}$, $1 \mathrm{H}, \mathrm{NHC} \underline{\mathrm{HCOOH}}), 4.45$ (s, 2H, pteridine-CH$\left.-\mathrm{N}), 6.50\left(\mathrm{~s}, 2 \mathrm{H}, \mathrm{C}_{4} \mathrm{~N}_{2}\right)_{2}\right), 6.90(\mathrm{~d}, 2 \mathrm{H}, \mathrm{N}-\mathrm{Ph}-$ $\left.(\underline{H})_{\text {ortho }}\right), 6.98(\mathrm{~s}, 1 \mathrm{H}, \underline{H \mathrm{~N}}-\mathrm{Ph}), 7.60-7.97(\mathrm{~m}, 7 \overline{\mathrm{H}}, \mathrm{N}-\mathrm{Ph}-(\underline{H})$ (meta) and pyrimidine-4-Ph, pyrimidine $\left.\mathrm{C}_{2} \underline{H}\right), 8.23(\mathrm{~s}, 1 \mathrm{H}, \mathrm{N} \underline{H C O}), 8.68\left(\mathrm{~s}, 1 \mathrm{H}\right.$, pteridine- $\left.\mathrm{C}_{7} \underline{H}\right), 11.39\left(\mathrm{~s}, 1 \mathrm{H}, \mathrm{CH}_{2} \mathrm{CH}_{2} \mathrm{COO} \underline{H}\right)$, $12.28\left(\mathrm{~s}, 1 \mathrm{H}, \mathrm{CH}_{2} \mathrm{COO} \underline{H}\right)$. Calcd. for $\mathrm{C}_{30} \mathrm{H}_{24} \mathrm{~N}_{10} \mathrm{O}_{6}$ (620.57): C, 58.06; H, 3.90; N, 22.57; found: $\mathrm{C}, 57.83 ; \mathrm{H}, 3.76 ; \mathrm{N}, 22.50$.

\subsection{Biological Activity (Sensitivity Tests) by Kirby-Bauer Method}

Antimicrobial activity of the tested samples was determined using a modified KirbyBauer disc diffusion method [39-44].

Supplementary Materials: The following are available online Spectral data as Supplementary Materials.

Author Contributions: Conceptualization, H.A.S. and O.A.A.A.; methodology, B.M.A.A.M.; software, H.A.S.; validation, H.A.S., O.A.A.A. and B.M.A.A.M.; formal analysis, H.A.S.; investigation, H.A.S.; resources, O.A.A.A.; data curation, H.A.S.; writing-original draft preparation, H.A.S.; writing-review and editing, O.A.A.A.; visualization, H.A.S.; supervision, O.A.A.A.; project administration, O.A.A.A.; funding acquisition, O.A.A.A. All authors have read and agreed to the published version of the manuscript.

Funding: This research was funded by Taif University Researchers Supporting Project number (TURSP-2020/220), Taif University, Taif, Saudi Arabia.

Institutional Review Board Statement: Not applicable.

Informed Consent Statement: Not applicable.

Acknowledgments: Taif University Researchers Supporting Project number (TURSP-2020/220), Taif University, Taif, Saudi Arabia.

Conflicts of Interest: The authors declare no conflict of interest.

Sample Availability: Samples of the compounds are available from the authors.

\section{References}

1. National Center for Biotechnology Information. PubChem Compound Database; CID $\frac{1}{4} 6037$. Available online: https:/ / pubchem. ncbi.nlm.nih.gov / compound / 6037 (accessed on 25 June 2016). 
2. Herbert, V.; Zalusky, R. Interrelations of vitamin B12 and folic acid metabolism: Folic acid clearance studies. J. Clin. Investig. 1962, 41, 1263-1276. [CrossRef] [PubMed]

3. Quinlivan, E.P.; McPartlin, J.; McNulty, H.; Ward, M.; Strain, J.J.; Weir, D.G.; Scott, J.M. Importance of both folic acid and vitamin $\mathrm{B} 12$ in reduction of risk of vascular disease. Lancet 2002, 359, 227-228. [CrossRef]

4. Pitkin, R.M. Folate and neural tube defects. Am. J. Clin. Nutr. 2007, 85, 285S-288S. [CrossRef] [PubMed]

5. Green, N.S. Folic Acid Supplementation and Prevention of Birth Defects. J. Nutr. 2002, 132, 2356S-2360S. [CrossRef]

6. Duthie, S.J. Folic acid deficiency and cancer: Mechanisms of DNA instability. Br. Med. Bull. 1999, 55, 578-592. [CrossRef]

7. Aleem, G.; Yibin, Z.; Mc John, J.; Roy, L.K. Synthesis of Classical and Nonclassical Partially Restricted Linear Tricyclic 5-Deaza Antifolates. J. Med. Chem. 2002, 45, 5173-5181.

8. Edward, C.T.; Jerauld, S.S.; Stephen, R.F. Synthesis of DL-7,10-ethano-5-deazaaminopterin and L-7,10-ethano-5-deazafolic acid. J. Org. Chem. 1985, 50, 1005-1010.

9. Joseph, I.D.; Pamela, H.C.; Roy, L.K.; Yvette, G.; Francis, M.S. Synthesis and antifolate properties of 10-alkyl-5,10-dideaza analogs of methotrexate and tetrahydrofolic acid. J. Med. Chem. 1990, 33, 673-677.

10. Kotake, Y.; Okauchi, T.; Iijima, A.; Yoshimatsu, K.; Nomura, H. Novel 6-5 fused ring heterocycle antifolates with potent antitumor activity: Bridge modifications and heterocyclic benzoyl isosters of 2,4-diamino-6,7-dihydro-5H-cyclopenta[d]pyrimidine antifolate. Chem. Pharm. Bull. (Tokyo) 1995, 43, 829-841. [CrossRef]

11. Andre, R.; Henry, B.; Joel, E.W.; Richard, G.M. 5-Deaza-7-desmethylene analogues of 510-methylene-5678-tetrahydrofolic acid and related compounds: Synthesis and in vitro biological activity. J. Heterocycl. Chem. 1994, 31, 1241-1250.

12. Bailey, L.B.; Rampersaud, G.C.; Kauwell, G.P.A. Folic Acid Supplements and Fortification Affect the Risk for Neural Tube Defects, Vascular Disease and Cancer: Evolving Science. J. Nutr. 2003, 133, 1961S-1968S. [CrossRef] [PubMed]

13. Arzeni, C.; Pérez, O.E.; LeBlanc, J.G.; Pilosof, A.M.R. Egg albumin-folic acid nanocomplexes: Performance as a functional ingredient and biological activity. J. Funct. Foods 2015, 18, 379-386. [CrossRef]

14. Kronenberg, G.; Colla, M.; Endres, M. Folic acid, neurodegenerative and neuropsychiatric disease. Curr. Mol. Med. 2009, 9, 315-323. [CrossRef] [PubMed]

15. Mao, X.; Xing, X.; Xu, R.; Gong, Q.; He, Y.; Li, S.; Wang, H.; Liu, C.; Ding, X.; Na, R.; et al. Folic acid and vitamins D and B12 correlate with homocysteine in Chinese patients with type-2 diabetes mellitus, hypertension, or cardiovascular disease. Medicine 2016, 95, e2652. [CrossRef]

16. Keser, I.; Ilich, J.Z.; Vrkic, N.; Giljevic, Z.; Colic Baric, I. Folic acid and vitamin $B_{12}$ supplementation lowers plasma homocysteine but has no effect on serum bone turnover markers in elderly women: A randomized, double-blind, placebo-controlled trial. Nutr. Res. 2013, 33, 211-219. [CrossRef]

17. Solini, A.; Santini, E.; Ferrannini, E. Effect of short-term folic acid supplementation on insulin sensitivity and inflammatory markers in overweight subjects. Int. J. Obes. 2006, 30, 1197-1202. [CrossRef]

18. Moens, A.L.; Champion, H.C.; Claeys, M.J.; Tavazzi, B.; Kaminski, P.M.; Wolin, M.S.; Borgonjon, D.J.; van Nassauw, L.; Haile, A.; Zviman, M.; et al. High-dose folic acid pretreatment blunts cardiac dysfunction during ischemia coupled to maintenance of high-energy phosphates and reduces postreperfusion injury. Circulation 2008, 117, 1810-1819. [CrossRef]

19. Saad, H. Synthesis of some pyridyloxymethyloxadiazoles, thiazoles and triazoles of expected pharmacological activity. Indian J. Chem. 1996, 35B, 980-984.

20. Saad, H.A.; Allimony, H.A.; El-Mariah, F.A.A. Synthesis and Antimicrobial Activity of Some Nitrogen Heterobicyclic Systems: Part II. Indian J. Chem. 1998, 37B, 1142-1148.

21. Allimony, H.A.; Saad, H.A.; El-Mariah, F.A.A. Synthesis and antimicrobial activity of some nitrogen heterobicyclic systems: Part I. Indian J. Chem. Sec. B Org. Med. Chem. 1999, 38, 445-451.

22. El-Mariah, F.A.A.; Saad, H.A.; Allimony, H.A.; Abdel-Rahman, R.M. Synthesis and Antimicrobial Activity of Some Nitrogen Heterobicyclic Systems: Part III. Indian J. Chem. 2000, 39B, 36-41.

23. Saad, H.A.; Moustafa, H.Y.; Assy, M.G.; Sayed, M.A. Functionlization and Heteroannelation of Ethyl 2-(4'-chlorophenyl)-4mercapto-6-methylpyrimidine-5-carboxylate. Bull. Korean Chem. Soc. 2001, 22, 311-314.

24. Saad, H.A.; Mokbil, M.N.; El-Gendy, A.M.; Haikal, A.Z. Synthesis of some glycosides of pyridinone derivatives. AFINIDAD 2002, 59, 277-280. [CrossRef]

25. Deeb, A.; Saad, H. Tetrazolo[1,5-b]pyridazine-8-carbohydrazide, Synthesis and some reactions. Heterocycles 2003, 60, 1873-1879. [CrossRef]

26. Moustafa, A.H.; Saad, H.A. Synthesis of Phenazone Derivatives. J. Chem. Res. 2005, 2005, 328-331. [CrossRef]

27. Saad, H.A.; Moustafa, A.H. Synthesis of Fused heteropolycyclic systems containing an indole moiety. J. Chem. Res. 2006, 2006, 318-323. [CrossRef]

28. Moustafa, A.H.; Saad, H.A.; Shehab, W.S.; El-Mobayed, M.M. Synthesis of Some New Pyrimidine Derivatives of Expected Antimicrobial Activity. Phosphorus Sulfur Silicon Relat. Elem. 2008, 183, 115-135. [CrossRef]

29. Saad, H.A.; Youssef, M.M.; Mosselhi, M.A. Microwave Assisted Synthesis of Some New Fused 1,2,4-Triazines Bearing Thiophene Moieties with Expected Pharmacological Activity. Molecules 2011, 16, 4937-4957. [CrossRef]

30. Saad, H.A.; Moustafa, A.H. Synthesis and Anticancer Activity of Some New S-Glycosyl and S-Alkyl 1,2,4-Triazinone Derivatives. Molecules 2011, 16, 5682-5700. [CrossRef] 
31. Saad, H.A. Synthesis of novel fused heterocyclic compounds from 7-amino-[1,2,4]triazino[3,4- $b][1,3,4]$ thiadiazine-8-carbonitrile. Curr. Org. Synth. 2012, 9, 573-582. [CrossRef]

32. Aly, M.R.E.; Gobouri, A.A.; Abdel Hafez, S.H.; Saad, H.A. Synthesis Reactions and Biological Activity of some Triazine Derivatives Containing Sulfa Drug Moieties. Russ. J. Bioorg. Chem. 2015, 4, 491-504.

33. Aly, M.R.E.; Saad, H.A.; Abdel Hafez, S.H. Three-Component Process for the Synthesis of Pyrimido[2,1-c][1,2,4]triazine Derivatives via Knoevenagel Condensation under Thermal Aqueous Conditions. Curr. Org. Synth. 2015, 12, 208-219.

34. Aly, M.R.E.; Saad, H.A.; Mohamed, M.A.M. Click reaction based synthesis, antimicrobial, and cytotoxic activities of new 1,2,3-triazoles. Bioorg. Med. Chem. Lett. 2015, 25, 2824-2830. [CrossRef] [PubMed]

35. Aly, M.R.E.; Saad, H.A.; Abdel Hafez, S.H. Synthesis, antimicrobial and cytotoxicity evaluation of new cholesterol congeners. Beilstein J. Org. Chem. 2015, 11, 1922-1932. [CrossRef]

36. Amin, M.A.; Saad, H.A. Synthesis and Biological Activity of Fused Heteropolycyclic Systems Containing an Indole Moiety. Curr. Org. Synth. 2016, 13, 116-125. [CrossRef]

37. Abdel-Rahmana, R.M.; Saad, H.A. Synthesis and Chemical Behavior of 1,2,4-Triazine Derivatives Bearing/Containing Phosphorus Amides Donor-Accepto Interactions-A Review. Curr. Org. Synth. 2016, 13, 408-425. [CrossRef]

38. El Azab, I.H.; Saad, H.A. Thiosemicarbazides, Potent Reagent for Synthesis of Some New 1,4-Diphenylbenzo[g]quinoxaline-5,10Dione Based Heterocycles. Heterocycles 2016, 92, 1833-1856. [CrossRef]

39. AlHarthi, R.R.; Ali, R.S.; Amina, M.A.; Saad, H.A. Synthesis of Some New Fused 1,2,4-Triazines of Expected Antimicrobial Activity. Russ. J. Gen. Chem. 2016, 86, 2906-2913.

40. Shehab, W.S.; Saad, H.A.; Mouneir, S.M. Synthesis and Antitumor/Antiviral Evaluation of 6-Thienyl-5-cyano-2- thiouracil Derivatives and Their Thiogalactosides Analogs. Curr. Org. Synth. 2017, 14, 291-298. [CrossRef]

41. Mohamed, M.A.M.; Abu-Alola, L.M.B.; Al-Zaidi, O.N.A.; Saad, H.A.H. Cyclocondensation Reactions of Hydrazonoyl Chlorides with Some Azines: Synthesis of New Fused Heterocycles of Expected Microbiological Activity. Int. J. Org. Chem. 2017, 7, 12-24. [CrossRef]

42. Ali, R.S.; Saad, H.A. Synthesis of Some Novel Fused Pyrimido $\left[4^{\prime \prime}, 5^{\prime \prime}: 5^{\prime}, 6^{\prime}\right][1,2,4]$ triazino- $\left[3^{\prime}, 4^{\prime}: 3,4\right][1,2,4]$ triazino $[5,6-b]$ indoles with Expected Anticancer Activity. Molecules 2018, 23, 693. [CrossRef] [PubMed]

43. Ali, R.S.; Saad, H.A. Synthesis and Pharmacological Studies of Unprecedented Fused Pyridazino $\left[3^{\prime}, 4^{\prime}: 5,6\right][1,2,4]$ triazino $[3,4-$ b][1,3,4]-thiadiazine Derivatives. Molecules 2018, 23, 1024. [CrossRef] [PubMed]

44. Bauer, A.W.; Kirby, W.M.; Sherris, C.; Turck, M. Antibiotic susceptibility testing by a standardized single disk method. Am. J. Clin. Pathol. 1966, 45, 493-496. [CrossRef] [PubMed]

45. Pfaller, M.A.; Burmeister, L.; Bartlett, M.A.; Rinaldi, M.G. Multicenter evaluation of four methods of yeast inoculum preparation. J. Clin. Microbiol. 1988, 26, 1437-1441. [CrossRef] [PubMed]

46. National Committee for Clinical Laboratory Standards. Performance Antimicrobial Susceptibility of Flavobacteria; National Committee for Clinical Laboratory Standards: Villanova, PA, USA, 1997; Volume 41.

47. National Committee for Clinical Laboratory Standards. Methods for Dilution Antimicrobial Susceptibility Tests for Bacteria that Grow Aerobically. Approved Standard M7-A3; National Committee for Clinical Laboratory Standards: Villanova, PA, USA, 1993.

48. Liebowitz, L.D.; Ashbee, H.R.; Evans, E.G.V.; Chong, Y.; Mallatova, N.; Zaidi, M.; Gibbs, D.; Global Antifungal Surveillance Group. A two-year global evaluation of the susceptibility of Candida species to fluconazole by disk diffusion. Diagn. Microbiol. Infect. Dis. 2001, 40, 27-33. [CrossRef]

49. Matar, M.J.; Ostrosky-Zeichner, L.; Paetznick, V.L.; Rodriguez, J.R.; Chen, E.; Rex, J.H. Correlation between E-test, disk diffusion, and microdilution methods for antifungal susceptibility testing of fluconazole and voriconazole. Antimicrob. Agents Chemother. 2003, 47, 1647-1651. [CrossRef] [PubMed] 